Submitted to The Astrophysical Journal

\title{
The First Catalog of Active Galactic Nuclei Detected by the Fermi Large Area Telescope
}

A. A. Abdo ${ }^{2,3}$, M. Ackermann ${ }^{4}$, M. Ajello ${ }^{4}$, A. Allafort ${ }^{4}$, E. Antolini ${ }^{5,6}$, W. B. Atwood ${ }^{7}$, M. Axelsson ${ }^{8,9}$, L. Baldini ${ }^{10}$, J. Ballet ${ }^{11}$, G. Barbiellini ${ }^{12,13}$, D. Bastieri ${ }^{14,15}$, B. M. Baughman ${ }^{16}$, K. Bechtol ${ }^{4}$, R. Bellazzini ${ }^{10}$, B. Berenji ${ }^{4}$, R. D. Blandford ${ }^{4}$, E. D. Bloom ${ }^{4}$, J. R. Bogart ${ }^{4}$, E. Bonamente ${ }^{5,6}$, A. W. Borgland ${ }^{4}$, A. Bouvier ${ }^{4}$, J. Bregeon ${ }^{10}$, A. Brez ${ }^{10}$, M. Brigida ${ }^{17,18}$, P. Bruel ${ }^{19}$, R. Buehler ${ }^{4}$, T. H. Burnett ${ }^{20}$, S. Buson ${ }^{14}$, G. A. Caliandro ${ }^{21}$, R. A. Cameron ${ }^{4}$, A. Cannon ${ }^{22,23}$, P. A. Caraveo ${ }^{24}$, S. Carrigan ${ }^{15}$, J. M. Casandjian ${ }^{11}$, E. Cavazzuti ${ }^{1,25}$, C. Cecchi ${ }^{5,6}$, Ö. Çelik ${ }^{22,26,27}$, A. Celotti ${ }^{28}$, E. Charles ${ }^{4}$, A. Chekhtman ${ }^{2,29}$, A. W. Chen ${ }^{24}$, C. C. Cheung ${ }^{2,3}$, J. Chiang ${ }^{4}$, S. Ciprini ${ }^{6}$, R. Claus ${ }^{4}$, J. Cohen-Tanugi ${ }^{30}$, J. Conrad ${ }^{31,9,32}$, L. Costamante ${ }^{4}$, G. Cotter ${ }^{33}$, S. Cutini ${ }^{25}$, V. D'Elia ${ }^{25}$, C. D. Dermer ${ }^{2}$, A. de Angelis ${ }^{34}$, F. de Palma ${ }^{17,18}$, A. De Rosa ${ }^{35}$, S. W. Digel ${ }^{4}$, E. do Couto e Silva ${ }^{4}$, P. S. Drell ${ }^{4}$, R. Dubois ${ }^{4}$, D. Dumora ${ }^{36,37}$, L. Escande ${ }^{36,37}$, C. Farnier ${ }^{30}$, C. Favuzzi ${ }^{17,18}$, S. J. Fegan ${ }^{19}$, E. C. Ferrara ${ }^{22}$, W. B. Focke ${ }^{4}$, P. Fortin ${ }^{19}$, M. Frailis ${ }^{34,38}$, Y. Fukazawa ${ }^{39}$, S. Funk ${ }^{4}$, P. Fusco ${ }^{17,18}$, F. Gargano ${ }^{18}$,

D. Gasparrini ${ }^{1,25}$, N. Gehrels ${ }^{22,40,41}$, S. Germani ${ }^{5,6}$, B. Giebels ${ }^{19}$, N. Giglietto ${ }^{17,18}$, P. Giommi ${ }^{25}$, F. Giordano ${ }^{17,18}$, M. Giroletti ${ }^{42}$, T. Glanzman ${ }^{4}$, G. Godfrey ${ }^{4}$, P. Grandi ${ }^{43}$, I. A. Grenier ${ }^{11}$, M.-H. Grondin ${ }^{36,37}$, J. E. Grove ${ }^{2}$, S. Guiriec ${ }^{44}$, D. Hadasch ${ }^{45}$, A. K. Harding ${ }^{22}$, M. Hayashida ${ }^{4}$,

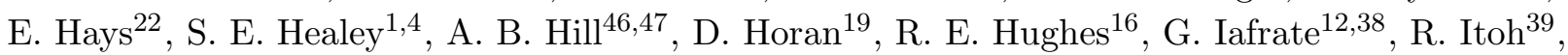
G. Jóhannesson ${ }^{4}$, A. S. Johnson ${ }^{4}$, R. P. Johnson ${ }^{7}$, T. J. Johnson ${ }^{22,41}$, W. N. Johnson ${ }^{2}$,

T. Kamae ${ }^{4}$, H. Katagiri ${ }^{39}$, J. Kataoka ${ }^{48}$, N. Kawai ${ }^{49,50}$, M. Kerr ${ }^{20}$, J. Knödlseder ${ }^{51}$, M. Kuss ${ }^{10}$, J. Lande ${ }^{4}$, L. Latronico ${ }^{10}$, C. Lavalley ${ }^{30}$, M. Lemoine-Goumard ${ }^{36,37}$, M. Llena Garde ${ }^{31,9}$, F. Longo ${ }^{12,13}$, F. Loparco ${ }^{17,18}$, B. Lott ${ }^{1,36,37}$, M. N. Lovellette ${ }^{2}$, P. Lubrano ${ }^{5,6}$, G. M. Madejski ${ }^{4}$, A. Makeev ${ }^{2,29}$, G. Malaguti ${ }^{43}$, E. Massaro ${ }^{52}$, M. N. Mazziotta ${ }^{18}$, W. McConville ${ }^{22,41}$, J. E. McEnery ${ }^{22,41}$, S. McGlynn ${ }^{53,9}$, P. F. Michelson ${ }^{4}$, W. Mitthumsiri ${ }^{4}$, T. Mizuno ${ }^{39}$, A. A. Moiseev ${ }^{26,41}$, C. Monte ${ }^{17,18}$, M. E. Monzani ${ }^{4}$, A. Morselli ${ }^{54}$, I. V. Moskalenko ${ }^{4}$, S. Murgia $^{4}$, P. L. Nolan ${ }^{4}$, J. P. Norris ${ }^{55}$, E. Nuss ${ }^{30}$, M. Ohno ${ }^{56}$, T. Ohsugi ${ }^{57}$, N. Omodei ${ }^{4}$, E. Orlando ${ }^{58}$, J. F. Ormes ${ }^{55}$, M. Ozaki ${ }^{56}$, D. Paneque ${ }^{4}$, J. H. Panetta ${ }^{4}$, D. Parent ${ }^{2,29,36,37}$, V. Pelassa ${ }^{30}$, M. Pepe ${ }^{5,6}$, M. Pesce-Rollins ${ }^{10}$, S. Piranomonte ${ }^{59}$, F. Piron ${ }^{30}$, T. A. Porter ${ }^{4}$, S. Rainò ${ }^{17,18}$, R. Rando ${ }^{14,15}$, M. Razzano ${ }^{10}$, A. Reimer ${ }^{60,4}$, O. Reimer ${ }^{60,4}$, T. Reposeur ${ }^{36,37}$, J. Ripken ${ }^{31,9}$, S. Ritz ${ }^{7}$, A. Y. Rodriguez ${ }^{21}$, R. W. Romani ${ }^{4}$, M. Roth ${ }^{20}$, F. Ryde ${ }^{53,9}$, H. F.-W. Sadrozinski ${ }^{7}$, D. Sanchez ${ }^{19}$, A. Sander ${ }^{16}$, P. M. Saz Parkinson ${ }^{7}$, J. D. Scargle ${ }^{61}$, C. Sgrò ${ }^{10}$, M. S. Shaw ${ }^{4}$, E. J. Siskind ${ }^{62}$, P. D. Smith ${ }^{16}$, G. Spandre ${ }^{10}$, P. Spinelli ${ }^{17,18}$, J.-L. Starck ${ }^{11}$, E. Stawarz ${ }^{4,63}$, M. S. Strickman ${ }^{2}$, D. J. Suson ${ }^{64}$, H. Tajima ${ }^{4}$, H. Takahashi ${ }^{57}$, T. Takahashi ${ }^{56}$, T. Tanaka ${ }^{4}$,

G. B. Taylor ${ }^{65}$, J. B. Thayer ${ }^{4}$, J. G. Thayer ${ }^{4}$, D. J. Thompson ${ }^{22}$, L. Tibaldo ${ }^{14,15,11,66}$, D. F. Torres ${ }^{45,21}$, G. Tosti ${ }^{1,5,6}$, A. Tramacere ${ }^{4,67,68}$, P. Ubertini ${ }^{35}$, Y. Uchiyama ${ }^{4}$, T. L. Usher ${ }^{4}$, V. Vasileiou ${ }^{26,27}$, N. Vilchez ${ }^{51}$, M. Villata ${ }^{69}$, V. Vitale ${ }^{54,70}$, A. P. Waite ${ }^{4}$, E. Wallace $^{20}$, P. Wang $^{4}$, B. L. Winer ${ }^{16}$, K. S. Wood ${ }^{2}$, Z. Yang ${ }^{31,9}$, T. Ylinen ${ }^{53,71,9}$, M. Ziegler ${ }^{7}$ 


\footnotetext{
${ }^{1}$ Corresponding authors: S. E. Healey, sehealey@astro.stanford.edu; B. Lott, lott@cenbg.in2p3.fr; G. Tosti, Gino.Tosti@pg.infn.it; E. Cavazzuti, elisabetta.cavazzuti@asdc.asi.it; D. Gasparrini, gasparrini@asdc.asi.it

${ }^{2}$ Space Science Division, Naval Research Laboratory, Washington, DC 20375, USA

${ }^{3}$ National Research Council Research Associate, National Academy of Sciences, Washington, DC 20001, USA

${ }^{4}$ W. W. Hansen Experimental Physics Laboratory, Kavli Institute for Particle Astrophysics and Cosmology, Department of Physics and SLAC National Accelerator Laboratory, Stanford University, Stanford, CA 94305, USA

${ }^{5}$ Istituto Nazionale di Fisica Nucleare, Sezione di Perugia, I-06123 Perugia, Italy

${ }^{6}$ Dipartimento di Fisica, Università degli Studi di Perugia, I-06123 Perugia, Italy

${ }^{7}$ Santa Cruz Institute for Particle Physics, Department of Physics and Department of Astronomy and Astrophysics, University of California at Santa Cruz, Santa Cruz, CA 95064, USA

${ }^{8}$ Department of Astronomy, Stockholm University, SE-106 91 Stockholm, Sweden

${ }^{9}$ The Oskar Klein Centre for Cosmoparticle Physics, AlbaNova, SE-106 91 Stockholm, Sweden

${ }^{10}$ Istituto Nazionale di Fisica Nucleare, Sezione di Pisa, I-56127 Pisa, Italy

${ }^{11}$ Laboratoire AIM, CEA-IRFU/CNRS/Université Paris Diderot, Service d'Astrophysique, CEA Saclay, 91191 Gif sur Yvette, France

${ }^{12}$ Istituto Nazionale di Fisica Nucleare, Sezione di Trieste, I-34127 Trieste, Italy

${ }^{13}$ Dipartimento di Fisica, Università di Trieste, I-34127 Trieste, Italy

${ }^{14}$ Istituto Nazionale di Fisica Nucleare, Sezione di Padova, I-35131 Padova, Italy

${ }^{15}$ Dipartimento di Fisica "G. Galilei", Università di Padova, I-35131 Padova, Italy

${ }^{16}$ Department of Physics, Center for Cosmology and Astro-Particle Physics, The Ohio State University, Columbus, $\mathrm{OH} 43210$, USA

${ }^{17}$ Dipartimento di Fisica "M. Merlin" dell'Università e del Politecnico di Bari, I-70126 Bari, Italy

${ }^{18}$ Istituto Nazionale di Fisica Nucleare, Sezione di Bari, 70126 Bari, Italy

${ }^{19}$ Laboratoire Leprince-Ringuet, École polytechnique, CNRS/IN2P3, Palaiseau, France

${ }^{20}$ Department of Physics, University of Washington, Seattle, WA 98195-1560, USA

${ }^{21}$ Institut de Ciencies de l'Espai (IEEC-CSIC), Campus UAB, 08193 Barcelona, Spain

${ }^{22}$ NASA Goddard Space Flight Center, Greenbelt, MD 20771, USA

${ }^{23}$ University College Dublin, Belfield, Dublin 4, Ireland

${ }^{24}$ INAF-Istituto di Astrofisica Spaziale e Fisica Cosmica, I-20133 Milano, Italy

${ }^{25}$ Agenzia Spaziale Italiana (ASI) Science Data Center, I-00044 Frascati (Roma), Italy

${ }^{26}$ Center for Research and Exploration in Space Science and Technology (CRESST) and NASA Goddard Space Flight Center, Greenbelt, MD 20771, USA

${ }^{27}$ Department of Physics and Center for Space Sciences and Technology, University of Maryland Baltimore County, Baltimore, MD 21250, USA

${ }^{28}$ Scuola Internazionale Superiore di Studi Avanzati (SISSA), 34014 Trieste, Italy
} 


\footnotetext{
${ }^{29}$ George Mason University, Fairfax, VA 22030, USA

${ }^{30}$ Laboratoire de Physique Théorique et Astroparticules, Université Montpellier 2, CNRS/IN2P3, Montpellier, France

${ }^{31}$ Department of Physics, Stockholm University, AlbaNova, SE-106 91 Stockholm, Sweden

${ }^{32}$ Royal Swedish Academy of Sciences Research Fellow, funded by a grant from the K. A. Wallenberg Foundation

${ }^{33}$ Astrophysics, Oxford University, Oxford OX1 3RH, UK

${ }^{34}$ Dipartimento di Fisica, Università di Udine and Istituto Nazionale di Fisica Nucleare, Sezione di Trieste, Gruppo Collegato di Udine, I-33100 Udine, Italy

${ }^{35}$ INAF-Istituto di Astrofisica Spaziale e Fisica Cosmica, I-00133 Roma, Italy

${ }^{36}$ CNRS/IN2P3, Centre d'Études Nucléaires Bordeaux Gradignan, UMR 5797, Gradignan, 33175, France

${ }^{37}$ Université de Bordeaux, Centre d'Études Nucléaires Bordeaux Gradignan, UMR 5797, Gradignan, 33175, France

${ }^{38}$ Osservatorio Astronomico di Trieste, Istituto Nazionale di Astrofisica, I-34143 Trieste, Italy

${ }^{39}$ Department of Physical Sciences, Hiroshima University, Higashi-Hiroshima, Hiroshima 739-8526, Japan

${ }^{40}$ Department of Astronomy and Astrophysics, Pennsylvania State University, University Park, PA 16802, USA

${ }^{41}$ Department of Physics and Department of Astronomy, University of Maryland, College Park, MD 20742, USA

${ }^{42}$ INAF Istituto di Radioastronomia, 40129 Bologna, Italy

${ }^{43}$ INAF-IASF Bologna, 40129 Bologna, Italy

${ }^{44}$ Center for Space Plasma and Aeronomic Research (CSPAR), University of Alabama in Huntsville, Huntsville, AL 35899, USA

${ }^{45}$ Institució Catalana de Recerca i Estudis Avançats (ICREA), Barcelona, Spain

${ }^{46}$ Université Joseph Fourier - Grenoble 1 / CNRS, laboratoire d'Astrophysique de Grenoble (LAOG) UMR 5571, BP 53, 38041 Grenoble Cedex 09, France

${ }^{47}$ Funded by contract ERC-StG-200911 from the European Community

${ }^{48}$ Research Institute for Science and Engineering, Waseda University, 3-4-1, Okubo, Shinjuku, Tokyo, 169-8555 Japan

${ }^{49}$ Department of Physics, Tokyo Institute of Technology, Meguro City, Tokyo 152-8551, Japan

${ }^{50}$ Cosmic Radiation Laboratory, Institute of Physical and Chemical Research (RIKEN), Wako, Saitama 351-0198, Japan

${ }^{51}$ Centre d'Étude Spatiale des Rayonnements, CNRS/UPS, BP 44346, F-30128 Toulouse Cedex 4, France

${ }^{52}$ Università di Roma "La Sapienza", I-00185 Roma, Italy

${ }^{53}$ Department of Physics, Royal Institute of Technology (KTH), AlbaNova, SE-106 91 Stockholm, Sweden

${ }^{54}$ Istituto Nazionale di Fisica Nucleare, Sezione di Roma "Tor Vergata", I-00133 Roma, Italy

${ }^{55}$ Department of Physics and Astronomy, University of Denver, Denver, CO 80208, USA

${ }^{56}$ Institute of Space and Astronautical Science, JAXA, 3-1-1 Yoshinodai, Sagamihara, Kanagawa 229-8510, Japan

${ }^{57}$ Hiroshima Astrophysical Science Center, Hiroshima University, Higashi-Hiroshima, Hiroshima 739-8526, Japan
} 


\begin{abstract}
We present the first catalog of active galactic nuclei (AGN) detected by the LAT, corresponding to 11 months of data collected in scientific operation mode. The First LAT AGN Catalog (1LAC) includes $671 \gamma$-ray sources located at high Galactic latitudes $\left(|b|>10^{\circ}\right)$ that are detected with a test statistic $(T S)$ greater than 25 and associated statistically with AGNs. Some LAT sources are associated with multiple AGNs, and consequently, the catalog includes 709 AGNs, comprising 300 BL Lacertae objects (BL Lacs), 296 flat-spectrum radio quasars (FSRQs), 41 AGNs of other types, and 72 AGNs of unknown type. We also classify the blazars based on their spectral energy distributions (SEDs) as archival radio, optical, and X-ray data permit. In addition to the formal 1LAC sample, we provide AGN associations for 51 low-latitude LAT sources and AGN "affiliations" (unquantified counterpart candidates) for 104 highlatitude LAT sources without AGN associations. The overlap of the 1LAC with existing $\gamma$-ray AGN catalogs (LBAS, EGRET, AGILE, Swift, INTEGRAL, TeVCat) is briefly discussed. Various properties - such as $\gamma$-ray fluxes and photon power law spectral indices, redshifts, $\gamma$-ray luminosities, variability, and archival radio luminosities - and their correlations are presented and discussed for the different blazar classes. We compare the $1 \mathrm{LAC}$ results with predictions regarding the $\gamma$-ray AGN populations, and we comment on the power of the sample to address the question of the blazar sequence.
\end{abstract}

Subject headings: gamma rays: observations — galaxies: active — galaxies: jets — BL

\footnotetext{
${ }^{58}$ Max-Planck Institut für extraterrestrische Physik, 85748 Garching, Germany

${ }^{59}$ Osservatorio Astronomico di Roma, 00040 Monte Porzio Catone, Italy

${ }^{60}$ Institut für Astro- und Teilchenphysik and Institut für Theoretische Physik, Leopold-Franzens-Universität Innsbruck, A-6020 Innsbruck, Austria

${ }^{61}$ Space Sciences Division, NASA Ames Research Center, Moffett Field, CA 94035-1000, USA

${ }^{62}$ NYCB Real-Time Computing Inc., Lattingtown, NY 11560-1025, USA

${ }^{63}$ Astronomical Observatory, Jagiellonian University, 30-244 Kraków, Poland

${ }^{64}$ Department of Chemistry and Physics, Purdue University Calumet, Hammond, IN 46323-2094, USA

${ }^{65}$ University of New Mexico, MSC07 4220, Albuquerque, NM 87131, USA

${ }^{66}$ Partially supported by the International Doctorate on Astroparticle Physics (IDAPP) program

${ }^{67}$ Consorzio Interuniversitario per la Fisica Spaziale (CIFS), I-10133 Torino, Italy

${ }^{68}$ INTEGRAL Science Data Centre, CH-1290 Versoix, Switzerland

${ }^{69}$ INAF, Osservatorio Astronomico di Torino, I-10025 Pino Torinese (TO), Italy

${ }^{70}$ Dipartimento di Fisica, Università di Roma "Tor Vergata", I-00133 Roma, Italy

${ }^{71}$ School of Pure and Applied Natural Sciences, University of Kalmar, SE-391 82 Kalmar, Sweden
} 
Lacertae objects: general

\section{Introduction}

The Gamma-ray Large Area Space Telescope was launched on 2008 June 11. It began its scientific operations two months later, and shortly thereafter, it was renamed the Fermi Gammaray Space Telescope. Its primary instrument is the Large Area Telescope (LAT; Atwood et al. 2009), the successor to the Energetic Gamma-Ray Experiment Telescope (EGRET) on board the Compton Gamma-Ray Observatory (Thompson et al. 1993). The LAT offers a major increase in sensitivity over EGRET and the Italian Space Agency's Astro-rivelatore Gamma a Immagini Leggero (AGILE; Tavani et al. 2008), allowing it to study the $\gamma$-ray sky in unprecedented detail. In sky survey mode (Fermi's main observing mode), the LAT observes the entire sky every 3 hours, providing effectively uniform exposure on the timescale of days.

One of the major scientific goals of the Fermi mission is to investigate high-energy emission in active galactic nuclei (AGNs). Although it is generally accepted that the $\gamma$-rays detected from blazars are emitted from collimated jets of charged particles moving at relativistic speeds (Blandford \& Rees 1978; Maraschi et al. 1992), open questions remain. The mechanisms by which the particles are accelerated, the precise site of the $\gamma$-ray emission, and the origin of AGN variability and the $\gamma$-ray duty cycle of blazars are not well understood. The physical reasons for the observational differences between radio-loud and radio-quiet AGNs and between FSRQs and BL Lacs are also unclear. LAT observations of blazars and other AGNs are already helping to address these and other issues. Several in-depth spectral and/or multiwavelength studies of specific blazars (e.g., Abdo et al. 2009b, h; Aharonian et al. 2009) and of non-blazar radio galaxies (Abdo et al. $2009 \mathrm{c}, \mathrm{d}, \mathrm{g})$ have been performed.

The high sensitivity and nearly uniform sky coverage of the LAT make it a powerful tool for investigating the properties of large populations. The first list of bright AGNs detected by the LAT, the LAT Bright AGN Sample (LBAS; Abdo et al. 2009a) included bright AGNs at high Galactic latitude $\left(|b|>10^{\circ}\right)$ detected with high significance $(T S>100$, or $\gtrsim 10 \sigma)$ during the first three months of scientific operation. This list comprised 58 FSRQs, 42 BL Lacs, two radio galaxies, and four AGNs of unknown type. Following the models used to describe the $\gamma$-ray spectra obtained with previous $\gamma$-ray observatories (e.g., Mattox et al. 1996), the early analysis reported in the LBAS was carried out by fitting the $\gamma$-ray spectra at energies above $200 \mathrm{MeV}$ using a simple power-law model. This analysis revealed a fairly distinct spectral separation between FSRQs and BL Lacs, with FSRQs having significantly softer spectra. The division between the two classes was found to be at power law index $\Gamma \approx 2.2$. It has been suggested (Ghisellini et al. 2009) that this separation results from different radiative cooling of the electrons due to distinct accretion regimes in the two blazar classes. The $\gamma$-ray spectral properties and time-resolved multifrequency spectral energy distributions of LBAS sources were further investigated in Abdo et al. (2010a,ji). 
Here, we report on a larger AGN sample detected after 11 months of scientific operations. The LAT first-year catalog (1FGL; Abdo et al. 2010b) contains a total of 1451 sources detected with $T S>25$, and 1043 of these are at high Galactic latitudes $\left(|b|>10^{\circ}\right)$. We present a catalog of the high-latitude 1FGL sources that are associated with blazars and other AGNs. We refer to this as the First LAT AGN Catalog (1LAC). In addition to the 1LAC, we also provide, where possible, AGN associations for low-latitude LAT sources and AGN "affiliations" - candidate counterparts for which a quantitative association probability could not yet be computed - for unassociated highlatitude sources.

In Section 2, we describe the observations by the LAT and the analysis that led to the first-year catalog. In Section 3, we explain the method for associating $\gamma$-ray sources with AGN counterparts in a statistically meaningful way, present the results of this method, and describe the two schemes for classifying 1 LAC AGNs. Section 4 provides a brief census of the 1LAC sample. Section 5 summarizes some of the properties of the 1LAC, including the $\gamma$-ray flux distribution, the $\gamma$-ray photon spectral index distribution, the $\gamma$-ray variability properties, the redshift distribution, and the $\gamma$-ray luminosity distribution. In Section 6, we describe the multiwavelength properties, from radio to $\mathrm{TeV}$, of the $1 \mathrm{LAC}$ AGNs. We discuss the implications of the $1 \mathrm{LAC}$ results in Section 7 and conclude in Section 8.

In the following, we use a $\Lambda$ CDM cosmology with values within $1 \sigma$ of the Wilkinson Microwave Anisotropy Probe (WMAP) results (Komatsu et al. 2009); in particular, we use $h=0.71, \Omega_{m}=$ 0.27 , and $\Omega_{\Lambda}=0.73$, where the Hubble constant $H_{0}=100 h \mathrm{~km} \mathrm{~s}^{-1} \mathrm{Mpc}^{-1}$. We also define the radio spectral indices such that $S(\nu) \propto \nu^{-\alpha}$.

\section{Observations with the Large Area Telescope - Analysis Procedures}

The $\gamma$-ray sources in the 1LAC are a subset of those in the 1FGL catalog; we summarize here the procedures used in producing the 1FGL catalog. The data were collected from 2008 August 4 to 2009 July 4, primarily with standard sky-survey observations. Only photons in the "Diffuse" event clas: 1 with energies in the range $0.1-100 \mathrm{GeV}$ were considered in this analysis in order to minimize contamination from misclassified cosmic rays (Atwood et al. 2009). This photon class is described in more detail (comparison to the class used in the Bright Source List (BSL; Abdo et al. 2009f) paper, systematic uncertainties) in Abdo et al. (2010b). To minimize contamination from $\gamma$-rays from the Earth's limb, photons with incident directions greater than $105^{\circ}$ from the local zenith were removed. In addition, time ranges during which the rocking angle of the LAT was greater than $43^{\circ}$ were excluded from the data set because the bright limb of the Earth entered the field of view. This rocking angle limit removed only a small fraction of the data, with the excluded time intervals occurring during occasional 5-hour pointed observations at larger rocking angles ( $\gamma$-ray

\footnotetext{
${ }^{1}$ See http://fermi.gsfc.nasa.gov/ssc/data/analysis/documentation/Cicerone/Cicerone_Data/LAT_DP.html .
} 
burst [GRB] afterglow searches) and during even briefer intervals related to Sun avoidance during survey mode observations. A few minutes were excised around two bright GRBs (GRB 080916C and GRB 090510). The few time intervals with poor data quality, flagged as anything other than "Good" in the pointing/live time history (FT2) files, were also excluded. The resulting data set includes $245.6 \mathrm{~d}$ of live time. The standard Fermi-LAT ScienceTools software packag $2^{2}$ (version v9r15p2) was used with the "P6_V3_DIFFUSE" set of instrument response functions.

The source detection step made use of two wavelet algorithms, mr_filter (Starck \& Pierre 1998) and PGWAVE (Ciprini et al. 2007), as well as tools that maximize a simplified likelihood function (pointfind; Abdo et al. 2010b) and that implement a minimum spanning tree algorithm (Campana et al. 2008). The intention in using a variety of algorithms to generate a list of "seed" positions for sources was to keep the source-detection step from being a limiting factor in the analysis. As described in the 1FGL paper, the algorithms were run independently for different energy ranges to find both soft- and hard-spectrum sources. Yet more seeds were introduced from the Roma-BZCAT (Massaro et al. 2009) and WMAP (Hinshaw et al. 2007; Giommi et al. 2009) catalogs if no nearby LAT seeds were present. The seeds were essentially candidate sources, and each was evaluated in detail in the subsequent steps of the 1FGL catalog analysis using the standard gtlike tool to arrive at the final list.

The Galactic diffuse background model consistently employed throughout the analysis is the currently recommended version (gll_iem_v02), publicly released through the Fermi Science Support Center 3 . The isotropic background (including the $\gamma$-ray diffuse and residual instrumental backgrounds) model was derived from an overall fit of the diffuse component over the $|b|>30^{\circ}$ sky. The Galactic diffuse model and corresponding isotropic spectrum are described in more detail in documentation available from the Fermi Science Support Center.

To evaluate the source significance, we used the maximum-likelihood algorithm implemented in gtlike. For the 1FGL catalog, a threshold of 25 was adopted for the test statistic (TS) from the gtlike likelihood analysis. Sources found to have $T S>25$ were included in the 1FGL catalog. This corresponds approximately to a minimum significance of $4.1 \sigma$. Figure 1 displays a sky map, in Galactic coordinates, of the flux limit for photon spectral index $\Gamma=2.2$ and $T S=25$. The anisotropy (about a factor of 2) is due to the non-uniform Galactic diffuse background and nonuniform exposure (mostly arising from the passage of the Fermi satellite through the South Atlantic Anomaly). For soft sources, source confusion decreases the sensitivity to some extent (Abdo et al. 2010b).

The flux, photon spectral index $(\Gamma)$, and test statistic of each source in the energy range 0.1-100 GeV were determined by analyzing regions of interest (ROI) typically $12^{\circ}$ in radius. The model of the ROI used to fit the data was built taking into account all the sources detected within a

\footnotetext{
${ }^{2}$ http://fermi.gsfc.nasa.gov/ssc/data/analysis/documentation/Cicerone/

${ }^{3}$ http://fermi.gsfc.nasa.gov/ssc/data/access/lat/BackgroundModels.html
} 
given ROI. The fluxes in five bands $(0.1-0.3,0.3-1,1-3,3-10$, and 10-100 GeV) were also evaluated, with the photon spectral index held fixed to the best fit over the whole interval. The energy flux was determined from these fluxes, resulting in better accuracy than would be obtained from the power-law fitted function. For hard sources, the test statistics provided by the $10-100 \mathrm{GeV}$ band can be appreciable, which represents a notable difference with respect to EGRET, for which the acceptance dropped sharply in this range. The departure of the spectrum from a power-law shape can be estimated via a simple $\chi^{2}$ test on the five fluxes, referred to as the curvature index (Abdo et al. 2010b).

A TS map - a 2-dimensional array of likelihood TS values evaluated at a finely spaced grid centered on the direction of a $\gamma$-ray source - was generated for each source using pointfit. TS values are determined for each grid position independently by maximum-likelihood fitting of a test point source. From the TS maps, elliptical fits to the $95 \%$ confidence source location contours were derived; for this, the decrease of the TS away from the maximum-likelihood position of a source is interpreted in terms of the $\chi^{2}$ distribution with two degrees of freedom (the coordinates of the test source). The semimajor and semiminor axes of these ellipses were multiplied by 1.1 to account for systematic errors, which were evaluated by comparing the measured positions of bright sources to the known positions. The fiducial $95 \%$ error radius (the geometric mean of the semimajor and semiminor axes) is plotted against TS (derived as described above) in Figure 2, For each source, a simple variability index was derived from the $\chi^{2}$ value of the monthly flux distribution with a $3 \%$ systematic uncertainty included.

\section{Source Associations}

Any procedure for associating a $\gamma$-ray source with a lower-energy counterpart necessarily relies on a spatial coincidence between the two. In the EGRET era, the problem of counterpart associations was made very difficult by the large $\gamma$-ray localization contours: the mean $95 \%$ error radius for sources in the 18-month EGRET sky survey (Fichtel et al. 1994) was 0.62. The LAT offers a great improvement in source localization, resulting in a mean $95 \%$ error radius of 0.15 for the high-latitude 1FGL sources. (For comparison to LBAS, which included only sources with TS > 100 and had a mean $95 \%$ error radius of 0.14 , the corresponding value for high-latitude $1 \mathrm{FGL}$ sources with $T S>100$ is 0.09 .)

However, the LAT localization accuracy is not good enough to permit the determination of a lower-energy counterpart based only on positional coincidence. A firm counterpart identification is asserted only if the variability detected by the LAT corresponds with variability at longer wavelengths. In practice, such identifications are made only for a few sources (see Table 7 in Abdo et al. $2010 \mathrm{~b}$ ). For the rest, we use a method for finding associations between LAT sources and AGNs based on the calculation of association probabilities using a Bayesian approach implemented in the gtsrcid tool included in the LAT Science Tools package and described in the BSL paper. 


\subsection{The Bayesian Association Method}

The Bayesian method (de Ruiter et al. 1977; Sutherland \& Saunders 1992), implemented by the gtsrcid tool in the LAT Science Tools, is similar to that used by Mattox et al. (2001) to associate EGRET sources with flat-spectrum radio sources. An earlier version of the method was used and described in the BSL and LBAS papers. A more complete description is given in Abdo et al. $(2010 \mathrm{~b})$, but we provide a basic summary here. The method uses Bayes's theorem to calculate the posterior probability that a source from a catalog of candidate counterparts is truly an emitter of $\gamma$-rays detected by the LAT. The significance of a spatial coincidence between a candidate counterpart from a catalog $C$ and a LAT-detected $\gamma$-ray source is evaluated by examining the local density of counterparts from $C$ in the vicinity of the LAT source. We can then estimate the likelihood that such a coincidence is due to random chance and establish whether the association is likely to be real. To each catalog $C$, we assign a prior probability, assumed for simplicity to be the same for all sources in $C$, for detection by the LAT. The prior probability for each catalog can be tuned to give the desired number of false positive associations for a given threshold on the posterior probability, above which the associations are considered reliable (see Section 3.2.1). We use a slightly different configuration of gtsrcid from that used for the 1FGL catalog. This allowed us to assign multiple associations to a single LAT source and to find associations with probabilities above $50 \%$ (compared with a threshold of $80 \%$ for the 1FGL associations).

Candidate counterparts were drawn from a number of source catalogs. The most important ones are the Combined Radio All-sky Targeted Eight GHz Survey (CRATES; Healev et al. 2007), the Candidate Gamma-Ray Blazar Survey (CGRaBS; Healey et al. 2008), and the Roma-BZCAT (Massaro et al. 2009). The CRATES catalog contains precise positions, $8.4 \mathrm{GHz}$ flux densities, and radio spectral indices for over 11,000 flat-spectrum sources over the entire $|b|>10^{\circ}$ sky. CGRaBS, a sample of the 1,625 CRATES sources with radio and X-ray properties most similar to the blazars in the Third EGRET Catalog (3EG; Hartman et al. 1999), provides optical magnitudes, optical classifications, and spectroscopic redshifts. Roma-BZCAT is a list of blazars compiled based on an accurate examination of data from the literature and currently includes over 2800 sources, all observed at radio and optical frequencies and showing the observational characteristics of blazars. A complete list of the source catalogs used by gtsrcid can be found in Abdo et al. (2010b).

The same association method can be used at low latitudes; most of the candidate counterparts

in this region are drawn from the VLBA Calibrator Survey (Beaslev et al. 2002; Fomalont et al. 2003; Petrov et al. 2005, 2006; Kovalev et al. 2007; Petrov et al. 2008). These associations are discussed in Section 3.2 .2 . 


\subsection{Association Results}

\subsubsection{The First LAT AGN Catalog (1LAC)}

The 1LAC comprises all high-latitude $\left(|b|>10^{\circ}\right)$ sources with an association from gtsrcid; the full catalog includes 709 AGN associations for 671 distinct 1FGL sources and is shown in Table 1. An AGN is in the "high-confidence" sample if and only if its association probability $P$ is at least $80 \%$; this sample contains 663 AGNs. An AGN is in the "clean" sample if and only if it has $P \geq 80 \%$, it is the sole AGN associated with the corresponding 1FGL $\gamma$-ray source (as indicated by an " $\mathrm{S}$ " in the last column of Table 1), and it is not "flagged" in the 1FGL catalog as exhibiting some problem or anomaly that casts doubt on its detection. This last criterion eliminates 12 sources from the clean sample: 1FGL J0217.8+7353, 1FGL J0258.0+2033, 1FGL J0407.5+0749, 1FGL J0433.5+3230, 1FGL J0539.4-0400, 1FGL J0540.9-0547, 1FGL J1424.5-7847, 1FGL J1702.7-6217, 1FGL J1727.9+5010, 1FGL J1938.2-3957, 1FGL J2212.9+0654, and 1FGL J2343.6+3437. All figures presented here are for the clean sample, which contains 599 AGNs.

The LBAS associations included one LAT source that was associated with two radio counterparts. Abdo et al. (2009a) noted that, as the number of LAT detections increased, source confusion was likely to be more of a problem, and indeed, the 1LAC includes 35 LAT sources that are associated with more than one AGN (for a total of 73 such associations). In cases of multiple associations, we list each counterpart separately in Table 1 and indicate them in the last column of the table.

The prefix "FRBA" in the column of AGN names refers to sources observed at $8.4 \mathrm{GHz}$ as part of VLA program AH996 ("Finding and Rejecting Blazar Associations for Fermi-LAT $\gamma$-ray Sources"). The prefix "CLASS" refers to sources from the Cosmic Lens All-Sky Survey (Myers et al. 2003; Browne et al. 2003).

Figure 3 shows the distribution of normalized angular separations between the $\gamma$-ray sources and their AGN counterparts. The solid curve corresponds to the expected distribution for true associations while the dashed curve represents the expected distribution for purely random associations. These results provide confidence that most of the associations found are real.

The association probabilities can be used to estimate the number of false positive associations. In a sample of $k$ sources with association probabilities $P_{i}$, the number of false positives is

$N_{\text {false }} \approx \sum_{i=1}^{k}\left(1-P_{i}\right)$. Among the 709 associations in the entire 1LAC, 30 are false, but of the 663 sources in the high-confidence list, there are only $\sim 14$ false positives, and only $\sim 11$ of the sources in the clean sample are falsely associated. Additionally, there should be less than one false positive among the 363 most likely associations in the whole catalog. 


\subsubsection{Low-Latitude AGNs}

A simple extrapolation, based on the global density of 1LAC sources on the sky and the solid angle subtended by the Galactic plane region $\left(|b|<10^{\circ}\right)$, indicates that the LAT should be detecting 150 AGNs at low Galactic latitudes. Diffuse radio emission, interloping Galactic point sources, and heavy optical extinction make the low-latitude sky a difficult region for AGN studies, and catalogues of AGNs and AGN candidates often avoid it partially or entirely. However, we are able to make associations with 51 low-latitude AGNs; these are presented in Table 2. Although the associations are considered valid, these sources have, in general, been studied much less uniformly and much less thoroughly than the high-latitude sources at virtually all wavelengths, so we do not include them as part of the 1LAC in order to keep them from skewing any of our analyses of the overall $\gamma$-ray AGN population.

\subsubsection{AGN "Affiliations"}

For many of the 1FGL sources that are not formally associated with AGNs in the 1LAC, it is still possible to find nearby AGNs or AGN candidates for which reliable association probabilities can not (yet) be computed but which show some indication that they may be the correct counterpart. For example, using the ASDC multifrequency tool 4 , we performed a visual inspection of the vicinity of each unassociated 1FGL source. We considered objects inside the $95 \%$ error ellipse that showed hints of any blazar properties, such as coincident radio and X-ray emission and indications in the literature of variability, polarization, etc. For some sources, an optical spectrum, often from the Sloan Digital Sky Survey (SDSS; Adelman-McCarthy et al. 2008), was available, allowing us to classify them as BL Lacs or FSRQs. These sources have been evaluated by a method based on the known $\log N-\log S$ relationships of several types of AGNs (FSRQs, BL Lacs, flat-spectrum radio sources, etc.). We also used the figure of merit methodology developed by Sowards-Emmerd et al. (2003) and employed in the assembly of the LBAS source list; although we have discovered some problems with the calibration of the probabilities calculated by this approach, most of the resulting AGN associations seem likely to be legitimate. Both of these methods are being studied more carefully, but we list all of the unquantified correspondences (which we call "affiliations") derived by them, along with some of the properties of the affiliated AGNs, in Table 3. In all, we find 109 AGN affiliations for 104 high-latitude LAT sources. We expect that future refinements to our association methods will allow us to turn many of these into true, quantitative associations.

\footnotetext{
${ }^{4}$ These tools are available from the ASDC web site at the following URLs: http://tools.asdc.asi.it/ and http://www.asdc.asi.it/.
} 


\subsection{Source Classification}

\subsubsection{Optical Classification}

We classify each AGN according to its optical spectrum where available. Blazars are assigned optical classifications either as flat-spectrum radio quasars (FSRQs) or BL Lacertae objects (BL Lacs) using the same scheme as for CGRaBS. In particular, following Stocke et al. (1991), Urry \& Padovani (1995), and Marchã et al. (1996), we classify an object as a BL Lac if the equivalent width (EW) of the strongest optical emission line is $<5 \AA$, the optical spectrum shows a $\mathrm{Ca}$ II $\mathrm{H} / \mathrm{K}$ break ratio $C<0.4$, and the wavelength coverage of the spectrum satisfies $\left(\lambda_{\max }-\lambda_{\min }\right) / \lambda_{\max }>1.7$ in order to ensure that at least one strong emission line would have been detected if it were present. Although other definitions of BL Lac objects are sometimes applied (e.g., using the EWs of the [O II] $\lambda 3727$ and [O III] $\lambda 5007$ lines and/or different limits on $C$; see, e.g., Landt et al. 2004), the definition used here can be applied over a large redshift range, with the caveat that high-redshift blazars may be classified as BL Lacs or FSRQs using different emission lines from those used for low-redshift objects. The classification of higher-redshift sources will preferentially use lines at shorter wavelengths (e.g., Ly $\alpha \lambda 1216$ and C IV $\lambda 1549$ ) than for low-redshift

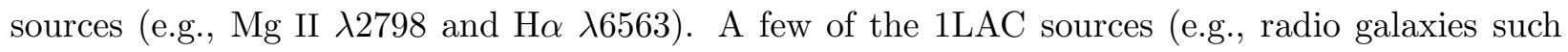
as Centaurus A and NGC 1275) that are not considered blazars are listed in Table 1 simply as "AGNs"; these objects are discussed individually in greater detail in the following section. Sources for which no optical spectrum was available or for which the optical spectrum was of insufficient quality to determine the optical classification are listed as being of unknown type. Some redshifts and source types given in Table 1 may differ from previously published results (generally from the NASA Extragalactic Database 5 [NED], SDSS, and/or Véron-Cetty \& Véron 2006). We thoroughly examined the data in the literature for accuracy and compatibility with our classification scheme, and our reevaluations of these results are reflected in Table 1.

A substantial number of the redshifts and optical classifications presented in Table 1 are from our own optical follow-up campaigns. Most of these come from spectroscopic observations conducted with the Marcario Low-Resolution Spectrograph (Hill et al. 1998) on the $9.2 \mathrm{~m}$ HobbyEberly Telescope (HET) at McDonald Observatory. Other facilities that have contributed optical results include the $3.6 \mathrm{~m}$ New Technology Telescope at La Silla, the $5 \mathrm{~m}$ Hale Telescope at Palomar, the $8.2 \mathrm{~m}$ Very Large Telescope at Paranal, and the $10 \mathrm{~m}$ Keck I Telescope at Mauna Kea. These spectra will be examined in greater detail in a subsequent paper (Shaw et al. 2010). A number of the HET redshifts were confirmed, and new results were obtained, with the $3.6 \mathrm{~m}$ Telescopio Nazionale Galileo at La Palma. This work will also be detailed in an upcoming publication (Piranomonte \& D'Elia 2010).

\footnotetext{
${ }^{5}$ http://nedwww.ipac.caltech.edu/
} 


\subsubsection{SED Classification}

The 1LAC blazars are also classified based on the peak of the synchrotron component of the broadband SED. In most cases, it is not possible to build a complete SED with simultaneous data, so we follow the scheme outlined by Abdo et al. (2010i). This scheme is an extension to all blazars of a standard classification system introduced by Padovani \& Giommi (1995) for BL Lacs. We estimate the frequency of the synchrotron peak, $\nu_{\text {peak }}^{\mathrm{S}}$, using the broadband spectral indices $\alpha_{\text {ro }}$ (between $5 \mathrm{GHz}$ and $5000 \AA$ ) and $\alpha_{\text {ox }}$ (between $5000 \AA$ and $1 \mathrm{keV}$ ). This method uses an analytic relationship calibrated on the $\nu_{\text {peak }}^{\mathrm{S}}$ values directly measured from the SEDs of the 48 sources studied by Abdo et al. (2010j), who confirm that these sources are representative of the global sample. This relationship is as follows:

$$
\log \nu_{\text {peak }}^{\mathrm{S}}= \begin{cases}13.85+2.30 X & \text { if } X<0 \text { and } Y<0.3, \\ 13.15+6.58 Y & \text { otherwise }\end{cases}
$$

where $X=0.565-1.433 \alpha_{\text {ro }}+0.155 \alpha_{\text {ox }}$ and $Y=1.000-0.661 \alpha_{\text {ro }}-0.339 \alpha_{\text {ox }}$. We use the estimated value of $\nu_{\text {peak }}^{\mathrm{S}}$ to classify the source as a low-synchrotron-peaked, or LSP, blazar (for sources with $\nu_{\text {peak }}^{\mathrm{S}}<10^{14} \mathrm{~Hz}$ ), an intermediate-synchrotron-peaked, or ISP, blazar (for $10^{14} \mathrm{~Hz}<\nu_{\text {peak }}^{\mathrm{S}}<$

$10^{15} \mathrm{~Hz}$ ), or a high-synchrotron-peaked, or HSP, blazar (for sources with $\nu_{\text {peak }}^{\mathrm{S}}>10^{15} \mathrm{~Hz}$ ). Figure4 displays the locations of BL Lacs from the clean sample in the $\left(\alpha_{\mathrm{ox}}, \alpha_{\mathrm{ro}}\right)$ plane. The data used for calculating the broadband spectral indices were obtained mainly from Swift-XRT observations, the NRAO VLA Sky Survey (NVSS; Condon et al. 1998), and the USNO-B1.0 catalog (Monet et al. 2003 ) and completed with data from Roma-BZCAT. It is evident from Figure 4 that $\gamma$-ray-selected BL Lacs cover the region in this plane typically occupied by blazars selected at other frequencies, such as radio and X-rays. Figure 5 shows the soft X-ray flux vs. the radio flux density at $1.4 \mathrm{GHz}$ for the blazars in the clean sample. As in LBAS, we find that the broadband properties of the 1LAC blazars are consistent with those of the parent populations of BL Lacs and FSRQs.

We note that the SED classification method assumes that the optical and X-ray fluxes come from the non-thermal emission without contamination from the disk or accretion. For blazars in which the thermal components are non-negligible, this method may lead to a significant overestimation of the position of $\nu_{\text {peak }}^{\mathrm{S}}$.

\section{1LAC Population Census}

Table 4 summarizes the breakdown of 1LAC sources by type for the full catalog, the highconfidence sample, and the clean sample. The fraction of blazars (BL Lacs and FSRQs) that are BL Lacs ( $51 \%$ for the high-confidence sample) is higher than in LBAS (39\%) and much higher than in 3EG (21\%). As discussed in Section 5.1, for a given significance, BL Lacs can typically be detected by the LAT at a lower flux than can FSRQs, which have softer spectra. The presence of one ISP-FSRQ and one HSP-FSRQ warrants comment. The existence of higher-peaked FSRQs has 
not been definitively proven yet. We stress that the SED classifications given here can be improved with simultaneous data, and for blazars in which the thermal components are non-negligible, it

may lead to a significant overestimation of $\nu_{\text {peak }}^{\mathrm{S}}$. Moreover, the average error on the $\gamma$-ray spectral indices is 0.14, so it can not be ruled out that the candidate ISP- and HSP-FSRQs actually have lower synchrotron peak frequencies and are consistent with the rest of the sample.

The distributions of synchrotron peak positions $\nu_{\text {peak }}^{\mathrm{S}}$ are shown in Figure 6 . The large fraction of HSPs in the 1LAC sample contrasts sharply with the results from the 3EG sample, in which most BL Lacs $(>80 \%)$ were of the LSP type. This is also a consequence of the dependence of the limiting flux on the spectral index (see Section 5.1 and Figure 9).

Figure 7 shows the sky locations of the sources in the clean sample. It is clear that the distribution is not isotropic; there are more sources in the northern galactic hemisphere than in the southern one. The Galactic latitude distributions for FSRQs and BL Lacs are shown in Figure 8 along with the corresponding distributions for the Roma-BZCAT and 1FGL. The anisotropy, defined as $\left(N_{+}-N_{-}\right) /\left(N_{+}+N_{-}\right)$, where $N_{+}$and $N_{-}$are the number of sources at $b>10^{\circ}$ and $b<-10^{\circ}$ respectively, amounts to $-4 \%\left(N_{+}=135, N_{-}=146\right)$ for high-confidence FSRQs and $18 \%\left(N_{+}=\right.$ $\left.171, N_{-}=120\right)$ for high-confidence BL Lacs. For comparison, the sources in the 1FGL catalog are very evenly distributed over the two hemispheres: 559 sources with $b>10^{\circ}$ and 550 sources with $b<-10^{\circ}$.

\subsection{Misaligned AGNs}

We have performed a search for possible associations between LAT detections and non-blazar radio-loud sources (i.e., radio galaxies and steep-spectrum radio quasars) using three main lowfrequency surveys: the 3CR catalog (Bennett 1962; Spinrad et al. 1985); its revised version, the 3CRR catalog (Laing et al. 1983); and the Molonglo Southern 4 Jy Sample (MS4; Burgess \& Hunstead 2006). These catalogs are flux limited (3CR: 9 Jy; 3CRR: 10.9 Jy; MS4: 4 Jy) and cover large portions of the northern (3CR, 3CRR) and southern (MS4) sky. In addition, because these surveys were conducted at low frequencies (3CR and 3CRR: $178 \mathrm{MHz}$; MS4: $408 \mathrm{MHz}$ ), they detect radio sources primarily in the relatively steep part of the synchrotron emission spectrum, which is generally associated with the extended lobes rather than the compact cores.

As a result, these catalogs are particularly appropriate for selecting AGNs with jets misaligned with respect to the line of sight. The sources in these surveys exhibit radio maps with resolved and possibly symmetrical structures and steep radio spectra $\left(\alpha_{\mathrm{r}}>0.5\right)$. At higher radio frequencies, the distinction between blazars and misaligned radio sources can be less sharp as the compact core emission (characterized by a flat or inverted spectrum) can emerge and dominate the optically thin synchrotron emission from extended regions. In addition to Cen A (Abdo et al. 2009a), NGC 1275 (Abdo et al. 2009a.c), and M 87 (Abdo et al. 2009d), this search has resulted in the classification of five LAT-detected sources as misaligned AGNs: three radio galaxies (NGC $1218=3 \mathrm{C} 78$, 
PKS 0625-35, and NGC 6251) and two steep-spectrum radio quasars (3C 207 and 3C 380). The properties of steep-spectrum radio sources detected by the LAT will be examined further in a future publication (Abdo et al. 2010c).

Five other 1LAC sources have flat or nearly flat radio spectra between $\sim 1 \mathrm{GHz}$ and $8.4 \mathrm{GHz}$ and strong emission lines in their optical spectra, but these lines are narrow, in contrast to the broad emission features seen in FSRQs. Under the standard AGN unification paradigm (Antonucci 1993; Urry \& Padovani 1995), this is interpreted as an indication that the jet axis is at a larger angle to the line of sight than for a typical FSRQ. This causes the broad-line region to be obscured by the dusty torus surrounding the central black hole, leaving only narrow lines in the resulting spectrum, and such sources are commonly described as narrow-line radio galaxies (NLRGs). The NLRGs appearing in the 1LAC are 4C +15.05, PKS 1106+023, CGRaBS J1330+5202, 4C +15.54, and CGRaBS J2250-2806.

\subsection{Radio-Quiet AGNs}

Radio-loud narrow-line Seyfert 1 galaxies have previously been shown to be emitters of $\gamma$-rays (PMN J0948+0022, Abdo et al.2009g; B2 0321+33B, PKS 1502+036, and PKS 2004-447, Abdo et al. 2009i). There is a tentative indication that the LAT may have detected radio-quiet Seyfert galaxies or radio-quiet quasars. Indeed, the $1 \mathrm{LAC}$ includes 10 radio-quiet AGN associations for LAT sources (RX J0008.0+1450, 1WGA 0405.6-1313, CXOMP J084045.2+131617, CXOMP J084054.3+131456, SDSS J112042.47+071311.5, CXOCY J113008.8-144737, SDSS J125257.95+525925.6, SDSS J143847.94+371342.7, QSO J150255.20-415430.2, and SDSS J155140.52+085226.1). In seven of these cases, however, the LAT source is also associated with at least one radio-loud AGN (a blazar or a radio galaxy) with higher probability, so the $\gamma$-ray emission is very likely attributable to the radio-loud source. 1FGL J1120.4+0710 is associated both with SDSS J112042.47+071311.5, a Seyfert 1 galaxy, and CRATES J1120+0704; although the latter is of unknown type, it is a radio-loud source (probably a blazar) and is the likely source of the $\gamma$-ray emission. The remaining two radio-quiet 1LAC associations, RX J0008.0+1450 and QSO J150255.20-415430.2, have association probabilities under 70\%. More data and further study will be necessary to establish whether these objects are indeed emitters of $\gamma$-rays.

\subsection{Notes on Individual Sources}

1FGL J0047.3-2512: This source is associated with NGC 253, a starburst galaxy previously detected by the LAT (Abdo et al. 2010h). It has for some time been designated a Seyfert galaxy in the Véron catalog (most recently in Véron-Cetty \& Véron 2006), but this classification is questionable

(see, e.g., Förster Schreiber et al. 2004). Engelbracht et al. (1998) determine that the LINER-like characteristics of NGC 253 can be attributed entirely to starburst activity. 
1FGL J0339.1-1734: This source is associated with PKS 0336-177. The optical spectrum from the final release of 6dFGS (Jones et al. 2004, 2009) shows no strong emission lines, but the Ca II $\mathrm{H} / \mathrm{K}$ break is too large to satisfy the criterion $C<0.4$ for classification as a BL Lac.

1FGL J0405.6-1309: This source is associated with PKS 0403-13 (an FSRQ) and with 1WGA J0405.6-1313. Mason et al. (2000) classify the latter simply as an "AGN" but provide the redshift $(z=0.226)$ based on the detection of three emission lines, including broad $\mathrm{H} \beta$. The source is radio-faint (undetected in NVSS) and likely a Seyfert galaxy.

1FGL J0627.3-3530: This source is associated with PKS 0625-35, which shows an FR-I radio morphology. However, Wills et al. (2004) suggest a BL Lac classification for this object. They also note that, in contrast with the other genuine FR-I sources in their sample, an adequate fit to the optical continuum of PKS 0625-35 required a nonthermal power law in addition to emission from the host galaxy.

1FGL J0645.5+6033: This source is associated with BZU J0645+6024, a source with broad emission lines but with a steep radio spectrum.

1FGL J0923.2+4121: This source is associated with B3 0920+416, which Falco et al. (1998) characterize as a late-type galaxy.

1FGL J0956.5+6938: This source is associated with M 82, also known as NGC 3034 and 3C 231, a starburst galaxy previously detected by the LAT (Abdo et al. 2010h). There are indications that it may host a weak AGN (Wills et al. 1999), though this is not confirmed.

1FGL J1202.9+6032: This source is associated with CRATES J1203+6031. Falco et al. (1998) describes it as an early-type galaxy; it is listed in NED as a LINER.

1FGL J1305.4-4928: This source is associated with NGC 4945, which is both a Seyfert 2 galaxy and a starburst galaxy. It is the third starburst galaxy seen by the LAT, and its detection is reported here for the first time. A more careful analysis will be required to determine whether the $\gamma$-ray emission comes from the starburst activity or the AGN or both.

1FGL J1307.0-4030: This source is associated with ESO 323-G77, a nearby $(z=0.015)$ Seyfert 1.2 galaxy detected also by Swift-BAT (see, e.g., Ajello et al. 2009 and Section 6.3). It exhibits a hard X-ray spectrum typical of radio-quiet AGN. There is little indication that the source is a starburst galaxy. If the $\gamma$-ray emission from 1FGL J1307.0-4030 truly comes from ESO 323-G77, then its origin might be connected to the central AGN; this hypothesis is being examined more closely.

1FGL J1641.0+1143: This source is associated with CRATES J1640+1144, described by Mitton et al. (1977) only as a "galaxy." Since the authors do classify other sources as BL Lacs, it seems likely that CRATES J1640+1144 is not a BL Lac.

1FGL J1647.4+4948: This source is associated with CGRaBS J1647+4950, which Falco et al. (1998) characterize as a late-type galaxy. 
1FGL J1724.0+4002: This source is associated with B2 1722+40. The optical spectrum from Vermeulen et al. (1996) shows narrow forbidden emission lines and absorption features from the host galaxy.

1FGL J1756.6+5524: This source is associated with BZB J1756+5522 (a BL Lac) and with CRATES J1757+5523. The optical spectrum of the latter from Caccianiga et al. (2002) shows strong absorption features from the host galaxy and a large Ca II H/K break. The authors describe it as a passive elliptical galaxy.

1FGL J2008.6-0419: This source is associated with 3C 407, a source with broad emission lines but with a fairly steep radio spectrum.

1FGL J2038.1+6552: This source is associated with NGC 6951, which has been classified as a Seyfert 2 galaxy and a LINER. It also has a circumnuclear ring of starburst activity.

1FGL J2204.6+0442: This source is associated with $4 \mathrm{C}+04.77$, which is often called a BL Lac in the literature. A spectrum from Véron-Cetty \& Véron (1993), however, shows that the object exhibits strong broad emission lines characteristic of Seyfert 1 galaxies.

\section{Properties of the 1LAC Sources}

\subsection{Flux and Photon Spectral Index Distributions}

As demonstrated in Abdo et al. (2010a), many bright LAT blazars (most notably FSRQs and some LSP-BL Lacs) exhibit breaks in their $\gamma$-ray spectra. Despite this caveat, determining a photon spectral index fitted over the whole band is useful as it does reflect the source's spectral hardness and can be obtained with reasonable accuracy even for fairly faint sources.

The 11-month average photon spectral index is plotted against the flux $(E>100 \mathrm{MeV})$ estimated from a power law fit in Figure 9 for blazars in the clean sample. As the figure shows, the limiting flux corresponding to $T S=25$ depends fairly strongly on the photon spectral index; the solid curve corresponds to a simple analytical estimate (Lott et al. 2007). This effect is discussed in greater detail in Abdo et al. (2010b). FSRQs (red circles in Figure 9) mostly cluster in the soft spectral index region, while BL Lacs (blue circles) primarily occupy the hard spectral index region, confirming the trend seen for the LBAS sources (Abdo et al. 2009a). This implies that the limiting flux is different for FSRQs and BL Lacs. The respective flux distributions are compared in Figure 10. The mean fluxes are $8.5 \times 10^{-8}$ photons $\mathrm{cm}^{-2} \mathrm{~s}^{-1}$ and $2.9 \times 10^{-8}$ photons $\mathrm{cm}^{-2} \mathrm{~s}^{-1}$ for FSRQs and BL Lacs respectively. The faintest BL Lacs are about a factor of 3 fainter than the faintest FSRQs.

The overall flux distribution (of FSRQs and BL Lacs combined) is compared to that measured by EGRET in Figure 11a. The high-flux ends of the two distributions are in reasonable agreement. The peak flux distributions (maximum flux in a $\approx 15$-day viewing period for EGRET vs. maximum 
monthly flux for the LAT) are compared in Figure 11b. The peak fluxes observed by EGRET are substantially higher than those observed for the brightest 1LAC sources; this is illustrated in Figures 11k and 11d, which show the peak flux vs. mean flux and the peak flux/mean flux ratio respectively. The effect of using two different time binnings to determine the peak flux (15 days for EGRET vs. 1 month for the LAT) has been studied for the bright LBAS sources; the 15-day peak flux is only $13 \%$ higher, on average, than the 1-month peak flux. Hence, the main reason for the different peak fluxes observed by EGRET and the LAT is probably the different time spans over which the observations were conducted (4.5 years for EGRET vs. 11 months for the LAT), enabling the sources to explore a wider range of different states during the EGRET era. Note also that the EGRET observations were often triggered by flaring alerts provided by other facilities, causing a bias that is not present for the LAT.

The photon spectral index distributions for the clean sample, shown in Figure 12, confirm the trend found for the LBAS sources (Abdo et al. 2009a) and already mentioned above, with a clear difference between FSRQs and BL Lacs. Only 7\% of FSRQs (17/231) have a photon spectral index $\Gamma<2.2$, and only two FSRQs have $\Gamma<2$ (with uncertainties of $\sim 0.2$ ). Thus, it is quite clearly established that LAT-detected FSRQs exhibit soft $\gamma$-ray spectra. The 1LAC BL Lac distribution is broader than that observed for LBAS; it shows a larger overlap with the FSRQ distribution. In Abdo et al. (2010a), the BL Lacs with $\Gamma>2.2$ were found to be mostly LSP sources. This trend is also confirmed for the 1LAC sources, as illustrated in Figure 13, which shows $\Gamma$ vs. $\nu_{\text {peak }}^{\mathrm{S}}$. Abdo et al. (2010a) also point out that the BL Lac photon spectral index distribution suffers from a bias since the high-spectral-index (i.e., soft) end is cut off somewhat due to the TS threshold, as seen in Figure 9, Figure 14 shows the photon spectral index distributions for the different blazar classes for sources with $F[E>100 \mathrm{MeV}]>3 \times 10^{-8}$ photons $\mathrm{cm}^{-2} \mathrm{~s}^{-1}$, above which the sample is essentially complete (see Figure 9). The completeness of the sample will be examined more carefully in an upcoming paper on the LAT blazar population (Abdo et al. 2010d).

The presence of a few very hard $(\Gamma<1.7)$ sources warrants comment. These are fairly lowsignificance sources $(T S<150)$, as is evident from Figure 15. Note that typical statistical error bars reach $0.1-0.2$ for all sources with TS in that range. Figure 12 also displays the photon spectral index distribution for sources with unknown types. This distribution overlaps with both those of FSRQs and BL Lacs, which seems to rule out that these sources belong preferentially to either class.

Since the flux of each source is evaluated in five different bands, the departure of the spectrum from a power-law (PL) shape can be estimated via a simple $\chi^{2}$ test, referred to as the curvature index (Abdo et al. 2010b). Figure 16 displays the curvature index vs. total flux for FSRQs and for the three BL Lac subclasses. The trend corroborates the findings reported in Abdo et al. (2010a), namely, that the spectra of bright FSRQs and LSP-BL Lacs show strong departures from a PL shape while those of HSP-BL Lacs are essentially compatible with PLs. Note that due to their harder spectra, any deviation from a PL behavior would be more easily visible for HSP-BL Lacs. Because of larger statistical uncertainties, no conclusion can be drawn from the $\chi^{2}$ test for fainter 
sources (a similar limitation affects the variability index and is described below).

\subsection{Variability}

One of the defining characteristics of AGNs is their variability, measured at all time scales and at all wavelengths. In the LBAS sample, 46 sources (35 FSRQs, 10 BL Lacs, and one blazar of unknown type) were flagged as variable based on the results of a $\chi^{2}$ test applied to weekly light curves covering the first three months of the LAT sky survey. Recently, Abdo et al. (2010e) detected variability in 64 LBAS sources by analyzing weekly light curves over a period of 11 months.

The electronic version of the 1FGL catalog provides the light curves for all sources over 11 time intervals of 30.37 days each in the energy range $100 \mathrm{MeV}-100 \mathrm{GeV}$. The procedure used to make these light curves is described in Abdo et al. (2010b). Our analysis of the light curves of the sources in the clean sample shows that only six sources (3C 454.3, PKS 1510-08, 3C 279, PKS 1502+106, 3C 273, and PKS 0235+164) have a maximum monthy flux $(E>100 \mathrm{MeV})$ greater than $10^{-6}$ photons $\mathrm{cm}^{-2} \mathrm{~s}^{-1}$ (see also Figure 11b). The number of sources with peak flux values above $10^{-6}$ photons $\mathrm{cm}^{-2} \mathrm{~s}^{-1}$ increases if we consider shorter time intervals, as illustrated by the 41 Astronomer's Telegrams issued by the LAT collaboration (see below), mostly related to sources that showed short flares that reached this flux level on the time scale of days.

The 1FGL catalog also lists a variability index $V$, obtained using a simple $\chi^{2}$ test, that can be used to determine the probability that a source is variable. Sources for which $V>23.21$ have a $99 \%$ probability (for 10 degrees of freedom) of being variable. Figure 17 shows the distribution of the variability index for blazars (BL Lacs and FSRQs) in the clean sample. 189 blazars in the clean sample are found to be variable (to the right of the vertical line in Figure 17); they comprise 129 FSRQs (68\%), 46 BL Lacs (24\%), five AGNs of other types (NGC 1275, B2 0321+33B, $4 \mathrm{C}+15.54$, B2 1722+40, and CGRaBS J2250-2806), and nine blazars of unknown type. The 46 variable BL Lacs include 24 LSPs, nine ISPs, 10 HSPs, and three BL Lacs (CRATES J0058+3311, CGRaBS J0211+1051, and CRATES J1303+2433) for which there are not enough multiwavelength data to permit an SED classification. Figure 18 shows the distribution of the photon spectral index for the variable blazars. This distribution is dominated by sources with $\Gamma>2.2$. These are quite bright sources observed at energies higher than the peak energies of their SEDs, where the amplitude of the variability is generally larger.

It is important to bear in mind that for a source to be labeled as variable on the basis of its variability index, it must be both intrinsically variable and sufficiently bright. Larger statistical flux uncertainties obtained for fainter sources lead to a reduction of the variability index for a given fractional flux variation. To illustrate this effect, the variability index is plotted against the flux in Figure 19 for FSRQs and BL Lacs. The curves display the evolution of the variability index for two sample sources (the FSRQ 3C 454.3 and the BL Lac object AO 0235+164) that would be observed for the same temporal variation but lower mean fluxes. As a result of this effect, fainter sources 
appear less variable than brighter sources simply because we can not measure their variability as well. This may also explain why only $\sim 17 \%$ of the 1LAC BL Lacs, which are generally fainter and harder than FSRQs, are found to be variable by this criterion. The histograms in Figure 17 are artificially broadened by the flux dependence of the variability index shown in Figure 19, Allowing for the behavior shown by the the trends for 3C 454.3 and AO $0235+164$, there is no strong evidence for a significant difference in the intrinsic variability of FSRQs and BL Lacs.

The LAT Collaboration routinely issues Astronomer's Telegrams (ATels) to alert the community to transient sources or sources that are exhibiting flaring states in order to encourage simultaneous multiwavelength follow-up. Through 2010 Jan 20, 43 1LAC sources (and one at low latitude) have been the subjects of ATels from the LAT team. They comprise 32 FSRQs (with redshifts as high as $z=2.534$, for B3 1343+451), 10 BL Lacs, and one AGN of another type (CGRaBS J2250-2806, a narrow-line radio galaxy).

\subsection{Redshift Distributions}

The redshift distributions for FSRQs and BL Lacs in the clean sample are presented in Figure20 along with the corresponding ones for the WMAP blazars (Hinshaw et al. 2007; Giommi et al. 2009), which constitute a flux-limited all-sky sample above $1 \mathrm{Jy}$ in the central WMAP observing band (Q band, $41 \mathrm{GHz}$ ). Note that only 121 out of 291 (42\%) high-confidence 1LAC BL Lacs have measured redshifts. This is notably worse than for LBAS, in which 29 out of 42 (69\%) BL Lacs had measured redshifts. The redshift distributions of $\gamma$-ray-detected blazars - both for FSRQs and for BL Lacs - are fairly similar to those for WMAP, peaking around $z=1$ for FSRQs and at a lower redshift for BL Lacs (the lowest-redshift 1LAC BL Lac has $z=0.030$ ). The highest redshift for a high-confidence 1LAC FSRQ is $z=3.10$.

The photon spectral index is plotted against redshift in Figure 21. For FSRQs, no significant evolution is visible. This behavior is compatible with what was previously observed for LBAS. The attenuation effect of the extragalactic background light (EBL) would tend to introduce spurious evidence of evolution (Chen et al. 2004), but the soft spectra of FSRQs and the common presence of spectral breaks at a few GeV (Abdo et al. 2010a) both minimize this effect. A stronger evolution is seen for BL Lacs: hard sources are mostly located at low redshifts, while most high-redshift sources are softer than average (though it is important to bear in mind that most BL Lacs do not have measured redshifts). This trend was less clear for the LBAS BL Lacs (Abdo et al. 2009a) due to lower statistics. Figure 22 displays the photon spectral index distributions for sources with $z<0.5$ (top) and $z>0.5$ (middle), which are clearly different. This provides some insight into the properties of BL Lacs without measured redshifts. The photon spectral index distributions of BL Lacs with measured redshifts and with unknown redshifts are shown in the bottom panel of Figure 22, The distribution of BL Lacs with unknown redshifts includes notably fewer hard sources than that for BL Lacs with known redshifts. A Kolmogorov-Smirnov (K-S) test yields a probability of $6 \times 10^{-3}$ that the two distributions are drawn from the same underlying population. 
The similarity of the distribution of BL Lacs with unknown redshifts to that of BL Lacs with $z>0.5$ (K-S probability of 0.54 that the two distributions are drawn from the same underlying population) supports the idea of some bias toward higher redshifts for this class of objects.

\subsection{Luminosity Distributions}

The $\gamma$-ray luminosity $L_{\gamma}$ is calculated as in Ghisellini et al. (2009):

$$
L_{\gamma}=4 \pi d_{L}^{2} \frac{S\left(E_{1}, E_{2}\right)}{(1+z)^{2-\Gamma}}
$$

where $d_{L}$ is the luminosity distance, $\Gamma$ is the photon spectral index, $S$ is the energy flux, and $E_{1}$ and $E_{2}$ are the lower and upper energy bounds (taken here to be $100 \mathrm{MeV}$ and $100 \mathrm{GeV}$ ) respectively. Implicit in this derivation is the assumption that $E_{2} \gg E_{1}$, which is satisfied for our calculations. Figure 23 shows $L_{\gamma}$ plotted against the source redshift. The curves represent approximate instrumental limits calculated for two photon indices, $\Gamma=1.8$ and $\Gamma=2.2$. The low-redshift FSRQs with $L_{\gamma}>10^{48} \mathrm{erg} \mathrm{s}^{-1}$ could potentially still be detected at redshifts $z>3.1$.

Figure 24 shows the photon spectral index plotted against the $\gamma$-ray luminosity. The Pearson correlation coefficient for the two parameters is 0.17. It is important to bear in mind two issues when interpreting this correlation: the higher flux limit for soft sources (see Figures 9 and 10) and the difference in redshift distributions between FSRQs and BL Lacs (see Figure 201). Given their relative softness (and thus, high flux limit), FSRQs are excluded from the soft-faint region. BL Lacs are also partly excluded because of their relatively low fluxes. Luminosity limits calculated for different redshifts (with the same approach as for the flux limit shown in Figure 9) are displayed for reference in Figure 24 and illustrate somewhat the effect of the Malmquist bias 6 .

\section{Multiwavelength Properties of 1LAC Sources}

\subsection{Sources Detected at TeV Energies}

Over the last two decades, ground-based $\gamma$-ray instruments operating in the "TeV" or veryhigh-energy (VHE; $E \gtrsim 100 \mathrm{GeV}$ ) regime have detected 32 AGNs, with the pace of discovery increasing significantly 7 as the latest generation of instruments - CANGAROO, H. E. S. S., MAGIC and VERITAS - has been commissioned. Of these $32 \mathrm{TeV}$ AGNs, the majority are BL Lacs (23 HSPs, three ISPs, and two LSPs), with the remainder comprising one FSRQ, two FR-I radio galax-

\footnotetext{
${ }^{6}$ In a flux-limited sample, sources located at larger distances appear more luminous than closer ones since fainter sources are below the detection threshold (Malmquist 1920).

${ }^{7}$ TeVCat (http://tevcat.uchicago.edu/) presents an up-to-date catalog of TeV sources.
} 
ies, and one AGN of unknown type. A detailed analysis of the TeV AGNs detected with Fermi during the first 5.5 months of operation is given by Abdo et al. (2009e, and see references to the TeV detections therein). Five new TeV AGNs have since been detected (1ES 0414+009, Hofmann \& Fegan 2009; PKS 0447-437, Raue et al. 2009; RBS 0413, Ong \& Fortin 2009; 1ES 0502+675, Ong 2009a; VER J0521+211, Ong 2009b). Two of these detections and one previous detection (PKS 1424+240; Acciari et al. 2010) were motivated directly by the detection of $\mathrm{GeV}$ emission with Fermi.

Of the TeV AGNs, the 28 listed in Table 5 are detected by Fermi as 1FGL sources, with a mean photon spectral index of $\left\langle\Gamma_{\mathrm{GeV}}\right\rangle=2.02 \pm 0.01$. Taking only the subsample of $25 \mathrm{GeV}-$ $\mathrm{TeV}$ BL Lacs, the mean index is $\left\langle\Gamma_{\mathrm{GeV}}\right\rangle=1.92 \pm 0.01\left(\sigma_{\Gamma}=0.26\right)$, compared with $2.07 \pm 0.01$ $\left(\sigma_{\Gamma}=0.28\right)$ for the larger sample of Fermi BL Lacs (see Figure 12b), illustrating that the TeV sources are among the hardest BL Lacs in the GeV regime. The measured spectra of the majority of the GeV-TeV BL Lacs are well described by power laws in both regimes. For many of the sources, the photon spectral index of the $\mathrm{GeV}$ emission differs significantly from that of the $\mathrm{TeV}$ emission, which may be an indication of the presence of a break in the $\gamma$-ray spectrum between the two regimes. However, most of the $\mathrm{TeV}$ spectra have not been measured simultaneously with the $\mathrm{GeV}$ spectra, and caution is advised when comparing the spectra in detail. The largest such break, consistent with $\Delta \Gamma \equiv \Gamma_{\mathrm{TeV}}-\Gamma_{\mathrm{GeV}} \sim 2$, is evident in the spectra of 1ES 1101+496, H 1426+428 and PG 1553+113. By contrast, the spectra of the nearby radio galaxies M 87 and Cen A show no evidence of a spectral break. The mean break index is $\langle\Delta \Gamma\rangle=1.3$. The values of $\Delta \Gamma$ for the $\mathrm{GeV}-\mathrm{TeV}$ sources are shown in Figure 25, plotted against the redshift of the source. Among the possible explanations for the apparent deficit of sources with small $\Delta \Gamma$ at high redshift is the effects of pair production with the EBL, which is expected to introduce a redshift-dependent steepening into the TeV spectra of extragalactic objects.

The four TeV AGNs not detected thus far by Fermi are RGB J0152+017, 1ES 0229+200, 1ES 0347-121 and PKS 0548-322, all HSP-BL Lacs. Extrapolating the measured power-law TeV

spectra from all the TeV AGNs down to $200 \mathrm{GeV}$, it is evident that these four have among the smallest fluxes of all TeV AGN at this energy.

\section{2. $\quad$ Sources Detected Previously at GeV Energies}

Of the 709 sources in the 1LAC, 114 sources were included in the BSL and were thus significantly detected (at $10 \sigma$ or greater) during the first three months of LAT observations. All of those sources were previously associated with AGNs as part of LBAS. Three low-confidence LBAS sources (0FGL J0909.7+0145, 0FGL J1248.7+5811, and 0FGL J1641.4+3939) are not confirmed in the $1 \mathrm{LAC}$ sample.

Ten years after EGRET, it is interesting to look at the fraction of the AGNs that were active in the EGRET era and are detected again by the LAT with a comparable flux. We consider two sources to be "positionally coincident" when the separation between their positions is less than the 
quadratic sum of their $95 \%$ error radii. In the 1LAC sample, 63 AGNs are positionally coincident with 3EG sources. Of these, the 3EG catalog lists 51 sources as AGN identifications and four as AGN associations. The 3EG catalog listed a total of 62 sources as AGN identifications, so there are 11 identified EGRET AGN that are not positionally coincident with 1LAC sources. 45 sources from the 1LAC have positions compatible with the revised EGRET catalog (EGR; Casandiian \& Grenier 2008), while 22 sources are positionally coincident with sources from the high-energy EGRET catalog (GEV; Lamb \& Macomb 1997). In all, 75 1LAC sources have coincident detections from one or more of these three EGRET catalogs.

11 of the sources in this catalog are included in the first year AGILE catalog (1AGL; Pittori et al. 2009). All 11 are identified as blazars by $A G I L E$, and indeed, these 11 comprise the total sample of blazars in the 1AGL catalog. Only two of these AGILE-detected sources do not have similar EGRET detections. In all, 77 1LAC sources have been cataloged by other GeV instruments.

These 77 sources are listed in Table 6 along with the mean fluxes and photon indices measured by the LAT and by EGRET. These AGNs are composed of 49 FSRQs, 21 BL Lacs, four AGNs of other types, and three AGNs of unknown types.

During its 4.5-year mission, EGRET found few sources with flux $(E>100 \mathrm{MeV})$ less than $10 \times 10^{-8}$ photons $\mathrm{cm}^{-2} \mathrm{~s}^{-1}$. A large number of the $1 \mathrm{LAC}$ sources have fluxes well below this value; such sources would not have been visible to EGRET. Some sources, such as 1FGL J0428.6-3756 (associated with PKS 0426-380) and 1FGL J2229.7-0832 (associated with PKS 2227-08), have flux values well above the EGRET threshold but were not seen by EGRET and yet are not noted as being variable in the 1FGL data. These examples continue to demonstrate that long-duration variability is evident in AGN $\gamma$-ray flux.

All of the EGRET sources seen at $10 \sigma$ significance and associated with flaring blazars have been detected by the LAT and appear with blazar associations.

\subsection{Sources Detected in the Hard X-ray Band}

In recent years, a new generation of hard X-ray telescopes has drastically improved our knowledge of the source populations in this energy band. The Swift-BAT and INTEGRAL-IBIS instruments have been conducting surveys at hard X-ray energies; the most recent catalogs are the fourth

IBIS catalog (Bird et al. 2010) of 723 sources detected at $17-100 \mathrm{keV}$ and the 54 -month Palermo BAT catalog (Cusumano et al. 2009) of 1049 sources 8 detected at 14-150 keV. These two telescopes perform in a complementary fashion; while IBIS is the more sensitive instrument with better angular resolution, it has a smaller field of view and has concentrated exposure in the Galactic plane. Conversely, the BAT instrument has lower instantaneous sensitivity but has a much larger field

ECusumano et al. (2009) describes the 39-month catalog; the 54-month catalog is available at: http://www.ifc.inaf.it/cgi-bin/INAF/pub.cgi?href=activities/bat/index.html. 
of view and a much more uniform exposure across the whole sky. An initial comparison between the LBAS sources and the fourth IBIS catalog by Ubertini et al. (2009) showed that only a small subset of the $>250$ INTEGRAL AGNs, of which 19 are blazars, was detected by the LAT.

Restricting the catalogs to $|b|>10^{\circ}$, there are 291 IBIS sources and 736 BAT sources. If we consider all of the 1LAC sources with associated counterparts that fall within the error circles of the hard X-ray catalogs, we find that 50 of the 1LAC sources can be associated with known hard X-ray sources. Of these 50 sources, one appears only in the fourth IBIS catalog, 16 appear in both the fourth IBIS catalog and the 54-month Palermo BAT catalog, and the remaining 35 are detected only in the 54-month Palermo BAT catalog. This is still a very small subset of AGNs despite the depth of the LAT catalog and the sky coverage of the BAT catalog.

Table 7 lists the high-confidence 1LAC AGNs detected in hard X-rays. This sample contains 27 FSRQs, 16 BL Lacs, and seven AGNs of other types. Most of the AGNs (54\%) detected in hard X-rays are FSRQs; this is expected since the external Compton peak of these high-luminosity objects reaches a maximum at $\mathrm{MeV}$ energies and declines in the hard X-rays, maintaining a substantial fraction of the emitting power. The difference in the spectral shapes of BL Lacs and FSRQs observed in hard X-rays indicates that this energy range probes the high-energy tail of the synchrotron peak in BL Lacs and the ascending part of the Compton peak in FSRQs.

Finally, there is evidence that the hard X-ray emission from most bright LAT blazars is, apparently, missed in spite of the sub-milliCrab sensitivity reached with INTEGRAL and Swift in the deepest fields.

\subsection{Radio Properties}

The high-latitude 1FGL sources associated with AGNs and presented here in the 1LAC are all radio sources at some level. The large number of sources found in CRATES can, by definition, be characterized as relatively bright, flat-spectrum radio sources. However, since a significant number of associations are found from other catalogs, it is interesting to contemplate the distributions of the flux densities, luminosities, and spectral indices.

In Figure 26, we plot the distribution of the radio flux density at $8.4 \mathrm{GHz}$ for the clean sample and separately for the FSRQs and the BL Lacs in this sample. The overall distribution, including also the blazars of unknown type and the other AGNs, has one main peak (corresponding to $\left.\left\langle S_{8 \mathrm{GHz}}, 1 \mathrm{LAC}\right\rangle \sim 900 \mathrm{mJy}\right)$. However, the FSRQs are on average brighter than the BL Lacs, with $\left\langle S_{8 \mathrm{GHz}}, \mathrm{FSRQ}\right\rangle \sim 1200 \mathrm{mJy}$ and $\left\langle S_{8 \mathrm{GHz}}, \mathrm{BLL}\right\rangle \sim 400 \mathrm{mJy}$.

In Figure 27, we plot the corresponding radio luminosities, calculated and $K$-corrected for the sources with measured redshifts. The distributions for FSRQ and BL Lacs are clearly different, with the FSRQs concentrated at higher radio luminosities $\left(\log \left(L_{r, \mathrm{FSRQ}}\left[\mathrm{erg} \mathrm{s}^{-1}\right]\right)=44.1 \pm 0.7 \mathrm{erg} \mathrm{s}^{-1}\right)$ and the BL Lacs distributed over a broad range $\left.\left(\log L_{r, \mathrm{BLL}}\left[\mathrm{erg} \mathrm{s}^{-1}\right]\right)=42.3 \pm 1.1 \mathrm{erg} \mathrm{s}^{-1}\right)$. This is 
similar to the distribution found in the LBAS, and the only sources with radio luminosities below $L_{r}=10^{40} \mathrm{erg} \mathrm{s}^{-1}$ are a few nearby AGNs (Cen A, NGC 253, and NGC 4945).

Finally, in Figure 28, we plot the spectral index distribution, calculated between either $1.4 \mathrm{GHz}$ from NVSS for sources with $\delta>-40^{\circ}$ or $0.84 \mathrm{GHz}$ from the Sydney University Molonglo Sky Survey (SUMSS; Mauch et al. 2003) for sources with $\delta<-40^{\circ}$ and $8.4 \mathrm{GHz}$ from CRATES (or other measurements from NED). The overall distribution is consistent with a flat spectral index ( $\alpha=0.08 \pm 0.32$ ). No difference is found between FSRQs and BL Lacs. Interestingly, a tail of sources with steeper spectral index is found, and it is mostly composed of non-blazars, such as radio galaxies. This suggests that $\gamma$-ray sources can also be associated with radio sources that are less closely aligned with the line of sight than blazars (see Section 4.1).

Since the different classes have different properties in the $\gamma$-ray band, the relationship between the radio emission and the high-energy emission merits a deeper discussion. A paper dedicated to this subject is in preparation, with a focus on the correlation between radio and $\gamma$-ray fluxes.

\section{Discussion}

Of the 1043 high-latitude $\left(|b|>10^{\circ}\right) \gamma$-ray sources in the 1FGL catalog, 671 are associated with 709 AGNs; these constitute the 1LAC. The 663 high-confidence AGN sources detected by Fermi in 11 months of data can be compared with the 66 high-confidence AGNs detected by EGRET at $>5 \sigma$ significance over its lifetime (Hartman et al. 1999). We estimate that a large fraction of the other 372 high-latitude sources are likely AGNs on the basis of their spectral and variability properties; indeed, we have found plausible AGN candidates for $\sim 100$ of them (see Section 3.2.3, In addition, $\sim 20$ known millisecond pulsars, and possibly a comparable number of yet unidentified millisecond pulsars, are among the remaining high-latitude 1FGL sources.

The 1 LAC represents a $\sim 5$-fold increase in the number of sources associated with $\gamma$-ray blazars over previously published source lists (in particular, the 106 high-confidence blazars in the LBAS catalog). It is likely that other blazars remain unidentified, as implied by the observed north-south anisotropy of the associated sources, which reflects the incompleteness of the counterpart AGN catalogs. This is partly supported by the similarity of the $\gamma$-ray properties, including flux and photon spectral index, of the unidentified sources to those of the 1LAC blazars (Abdo et al. 2010f). There are also indications from our early radio follow-up of the unidentified sources that the LAT is detecting blazars that are fainter in the radio than those appearing in the catalogs of flat-spectrum radio sources from which we draw candidate associations.

The overall properties of the 1LAC sources are generally consistent with those of the LBAS (Abdo et al. 2009a, 2010a,j), including observations of:

1. A small number of non-blazar sources.

The misaligned AGNs include six radio galaxies, of which only three (NGC 1275, Cen A, and 
M 87) were previously reported. Five of the six radio galaxies are low-power FR-I galaxies associated with LAT sources with very high probabilities $(P>98 \%)$. The sixth, NGC 6251, is a moderately powerful radio galaxy (Perley et al. 1984) and has a lower association probability. The misaligned FR-II radio galaxy $3 \mathrm{C} 111$ is associated with a LAT source (see Table 2), but it is not a member of the 1LAC since it resides at low Galactic latitude. In addition, two steep-spectrum radio quasars and five NLRGs are among the other non-blazar sources, though many of these have prominent radio cores and some lack extended radio emission.

2. Redshift distributions peaking at $z \approx 1$ for 1 LAC FSRQs and at low redshift for 1 LAC BL Lacs with known redshifts.

The maximum redshift of the high-confidence 1LAC FSRQs is $z=3.10$, larger than the maximum redshift $(z=2.286)$ for an EGRET blazar. For comparison, the maximum redshift in both CGRaBS and Roma-BZCAT is for an FSRQ with $z>5$. The redshift distribution for BL Lacs in the $1 \mathrm{LAC}$ with known redshift displays a broad low-redshift peak from the lowest known redshift (for Mkn 421) at $z=0.030$ to $z \approx 0.4$. The good agreement with the $W M A P$ redshift distribution for FSRQs, with a comparable number of sources, supports the idea that similar populations are being sampled by WMAP and the LAT. Both the FSRQ and BL Lac redshift distributions are compatible with the corresponding distributions from LBAS; the K-S probabilities that they derive from the same parent distributions are 0.99 and 0.89 for FSRQs and BL Lacs respectively.

No strong evidence for a new population of misaligned FSRQs emerging at lower redshifts is found. Other than NGC 6251, just mentioned, notably absent are detections, at least at the 1LAC significance level of $T S>25$, of nearby powerful FR-II radio galaxies such as Cyg A and Pic A, though the low-latitude FR-II radio galaxy 3C 111 is detected by the LAT.

3. A high BL Lac/FSRQ ratio, close to unity.

This ratio is even higher than that found for the LBAS sources, which comprise 42 BL Lacs and 57 FSRQs. It stands in sharp contrast to the population of 3EG blazars, in which FSRQs outnumber BL Lacs by a factor of 3 .

4. A high HSP/LSP ratio among BL Lacs.

The large HSP/LSP radio for BL Lacs is a result of the fact that the sample is significancelimited. The LAT detects hard-spectrum sources at higher significance than soft-spectrum sources with comparable photon number fluxes. Moreover, the LAT is far more sensitive to multi-GeV photons than was EGRET, which lost sensitivity above several GeV due to self-vetoing effects. Consequently, HSP-BL Lacs, with harder spectra than LSP-BL Lacs, are more prevalent in the $1 \mathrm{LAC}$ than in the $3 \mathrm{EG}$ catalog.

5. Little evidence for different variability properties for FSRQs and BL Lacs.

No significant differences in the variability of FSRQs and BL Lacs are observed for sources at comparable fluxes. Because HSP blazars are generally observed at low flux levels, however, 
the comparison of variability properties primarily concerns LSP/ISP-BL Lacs and FSRQs.

6. A fairly strong correlation between photon spectral index and blazar class for the detected sources.

FSRQs, which are almost all LSP blazars, are found to be soft, while BL Lacs, whether of the LSP, ISP, or HSP type, represent on average a population of harder-spectrum sources. The average photon spectral index $\langle\Gamma\rangle$ continuously shifts to lower values (i.e., harder spectra) as the class varies from FSRQs $(\langle\Gamma\rangle=2.48)$ to LSP-BL Lacs $(\langle\Gamma\rangle=2.28)$, ISP-BL Lacs $(\langle\Gamma\rangle=2.13)$, and HSP-BL Lacs $(\langle\Gamma\rangle=1.96)$.

Furthermore, as noted by Ghisellini et al. (2009) and seen in Figure 24, the $\gamma$-ray blazars detected with the Fermi-LAT separate into hard-spectrum, low-luminosity sources, primarily consisting of BL Lac objects, and high-luminosity, soft-spectrum sources, primarily consisting of FSRQs and LSP-BL Lacs. Compared with the LBAS sample, the blazar divide now displays an extension of the sample toward low-luminosity objects in each of the blazar subclasses. In addition, we identify a third branch consisting of radio galaxies, which are distinguished from the aligned blazars by their soft spectra and very low $\gamma$-ray luminosities.

The finding that FSRQs in the 1LAC almost all have soft $(\Gamma \gtrsim 2.2) \gamma$-ray spectra suggests a connection between the presence of strong emission lines and the nonthermal electron maximum Lorentz factor or spectral shape. It is consistent with scenarios (Ghisellini et al. 1998; Böttcher \& Dermer 2002) in which the strong ambient radiation fields, as revealed by the presence of emission lines, affects the acceleration and cooling of particles and controls the formation of the blazar SED. The luminosity dependence of the $\gamma$-ray spectral hardness is also consistent with the blazar sequence (Ghisellini et al. 1998; Fossati et al. 1998; Ghisellini \& Tavecchio 2008), in which the frequencies of the synchrotron and Compton peaks are inversely correlated with the apparent isotropic luminosities of the blazars. This behavior has been interpreted in terms of an evolution of FSRQs into BL Lac objects as the supply of matter fueling the accretion disk declines, the surrounding external radiation fields become less intense, and the jet weakens (Böttcher \& Dermer 2002; Cavaliere \& D'Elia 2002; Ghisellini \& Tavecchio 2008).

Important caveats must, however, be considered before drawing firm conclusions about the blazar sequence from the 1LAC sources. First, identification of the blazars used to construct the blazar sequence is biased by the flux limits and frequency ranges of the underlying radio and X-ray samples (Giommi et al. 1999; Padovani et al. 2003), and different conclusions about its validity can be drawn depending on the catalogs used to examine the sequence (Nieppola et al. 2006). Second, specific sources that do not conform to the sequence have been identified (Caccianiga \& Marchã 2004; Giommi et al. 2007). Third, the 1LAC is significance-limited rather than flux-limited, which results in preferential detection of lower-flux, harder-spectrum sources. This means that there is a strong bias against detecting weak, soft-spectrum $\gamma$-ray sources from low-luminosity LSP blazars that might violate the sequence. On the other hand, hard-spectrum HSP sources should be more easily detected, but there are no high-luminosity HSP sources detected in the LAT band (see 
Figure 24), as all high-luminosity objects have SEDs of the LSP type. This may, however, reflect the difficulty of measuring the redshifts of BL Lacs, despite efforts even with 10 m-class telescopes (Shaw et al. 2010). Even for sources associated with AGNs with high probabilities, a large fraction lack redshift measurements, including $58 \%$ of the high-confidence BL Lacs and $\sim 30$ BL Lacs with $\Gamma \lesssim 1.9$. Considering the recent results of Plotkin et al. (2010), who find that many BL Lacs might have redshifts larger than 2 (up to $z=4.9$ ), and the indication that BL Lacs with unknown redshifts may be found preferentially at higher redshift (see Section 5.3 ), it is possible that a number of distant, high-luminosity HSP-BL Lacs are included in the 1LAC sample. According to our SED classification, $\sim 20$ BL Lacs with unknown redshifts are already classified as HSPs. If they were all at high redshift (e.g., $z=2$ ), and excluding those already detected at very high energy (which indicates a low redshift due to a lack of EBL absorption), $\sim 7$ objects could have $\gamma$-ray luminosities $L_{\gamma} \gtrsim 10^{48} \mathrm{erg} \mathrm{s}^{-1}$.

The existence of such sources, though quite rare, would be contrary to expectations from the blazar sequence (Ghisellini et al. 1998; Fossati et al. 1998; Ghisellini \& Tavecchio 2008). In the absence of redshift measurements of possible high-luminosity, hard-spectrum sources, we can only conclude that the Fermi results seem compatible with the sequence. Identifying hard-spectrum blazar sources at high redshifts is also important for studies of the cosmological evolution of the extragalactic background light (Abdo et al. 2010g), which are hampered by their absence.

Related to this, it is interesting to compare the redshift distribution of the LAT blazars (in particular, the FSRQs) with that of the objects detected by BAT on Swift (Ajello et al. 2009). Despite a fourfold increase in exposure time with respect to the LBAS sample and a consideration of $\gamma$-ray sources with $T S>25$ in the 1LAC compared with $T S>100$ in LBAS, the number of LAT-detected blazars still drops sharply at $z \sim 2.5$, and only two of the high-confidence 1LAC blazars have $z>3$ (keeping in mind biases from the lack of redshift measurements). This is very different from the BAT survey, in which half of all FSRQs have $z>2$, and the distribution extends to $z \sim 4$. This behavior may be indicative of a shift in the SED peak frequencies toward lower values (i.e., a "redder" SED) for blazars at high redshifts. Indeed, the jets of the high-redshift BAT blazars are found to be more powerful than those of the LAT blazars and are among the most powerful known (Ghisellini et al. 2010). The peak of the inverse Compton flux for these objects, estimated to be in the $\mathrm{MeV}$ or even sub-MeV range, is located closer to the BAT band than to the LAT band, and the LAT instead samples the cutoff region of the inverse Compton spectrum.

Comparison of Fermi results with pre-launch GLAST expectations depends on how the 1LAC results are characterized. Except for a few dozen millisecond pulsars, nearly all of the 1043 highlatitude $\gamma$-ray sources in the 1FGL are likely to be AGNs, but only 671 high-latitude 1FGL sources are associated with AGNs. Adopting a radio/ $\gamma$-ray correlation, Stecker \& Salamon (1996) and Chiang \& Mukherjee (1998) predicted many thousands of blazars to a flux limit of $2 \times 10^{-9}$ photons $\mathrm{cm}^{-2} \mathrm{~s}^{-1}$ (for $E>100 \mathrm{MeV}$ ) reached after five years for a source with $\Gamma=2.1$, as did Narumoto \& Totani (2006), who treated the problem using radio and X-ray luminosity functions. Mücke \& Pohl (2000), using a physical blazar model matched to the EGRET data, predicted an 
increased fraction of BL Lac objects at fainter flux levels, with nearly 2000 BL Lacs and 1000 FSRQs at the one-year flux limit of $4 \times 10^{-9}$ photons $\mathrm{cm}^{-2} \mathrm{~s}^{-1}$ (for $E>100 \mathrm{MeV}$ ). Dermer (2007) predicted that $\sim 800$ FSRQs and $\sim 200$ BL Lacs would be detected after one year, also employing a physical blazar model. Assuming the validity of the blazar sequence, Inoue \& Totani (2009) predicted $\sim 600-1200$ blazars to a flux limit of $2 \times 10^{-9}$ photons $\mathrm{cm}^{-2} \mathrm{~s}^{-1}$ (for $E>100 \mathrm{MeV}$ ). However, predictions based on a minimum flux depend sensitively on the source spectral index, as shown by Figure 9, so more reliable predictions needed to take this effect into account.

The populations of AGNs that can be studied jointly at GeV and at $\mathrm{TeV}$ energies has increased dramatically beyond what could have been expected based on the experience of the EGRET era. There is now a large and increasing population of GeV-TeV AGNs that can be best studied through observations with both Fermi and the ground-based $\gamma$-ray observatories. Since its launch, the number of TeV AGNs detected also by Fermi has steadily increased as its exposure on these sources grows. Complementing this, the $\mathrm{TeV}$ observatories have started to observe the more promising Fermi AGNs, and a number of detections have resulted. One promising feature of joint $\mathrm{GeV}-\mathrm{TeV}$ observations is the power to infer the redshifts of BL Lacs from spectral modeling, as in the case of PG $1553+113$ (Abdo et al. 2010i).

\section{Conclusions}

We have presented the First LAT AGN Catalog (1LAC), consisting of 671 high-latitude $\gamma$-ray sources with $T S>25$ measured in the first 11 months of the Fermi-LAT sky survey. Due to multiple associations, these sources are associated with 709 AGNs. Besides a growing number of blazars and six radio galaxies, the extragalactic $\gamma$-ray sky now includes a small number of steepspectrum quasars and narrow-line radio galaxies. Confirming many of the LBAS results, we find a strong correlation between the 0.1-100 GeV $\gamma$-ray spectral index and the blazar type, whether defined in terms of optical emission line strength or peak frequency of the synchrotron component of the SED. The relationship between the $\gamma$-ray spectral properties and the spectral luminosity is complicated by selection biases for source identification and redshift determination, but the Fermi results seem compatible with the blazar sequence.

The First LAT AGN Catalog represents significant progress in terms of the number of detected sources, the diversity of source types, and the accuracy of measured $\gamma$-ray properties. This sample is likely to evolve in the future as more unidentified sources can be associated with AGNs thanks to better counterpart catalogs and continued correlated variability studies with more LAT observations. Bearing in mind the instrumental limitations described above, the 1LAC provides an essential foundation on which to build a much better understanding of the population of $\gamma$-rayemitting AGNs. 


\section{Acknowledgments}

The Fermi LAT Collaboration acknowledges generous ongoing support from a number of agencies and institutes that have supported both the development and the operation of the LAT as well as scientific data analysis. These include the National Aeronautics and Space Administration and the Department of Energy in the United States; the Commissariat à l'Energie Atomique and the Centre National de la Recherche Scientifique / Institut National de Physique Nucléaire et de Physique des Particules in France; the Agenzia Spaziale Italiana and the Istituto Nazionale di Fisica Nucleare in Italy; the Ministry of Education, Culture, Sports, Science and Technology (MEXT), High Energy Accelerator Research Organization (KEK) and Japan Aerospace Exploration Agency (JAXA) in Japan; and the K. A. Wallenberg Foundation, the Swedish Research Council and the Swedish National Space Board in Sweden.

Additional support for science analysis during the operations phase is gratefully acknowledged from the Istituto Nazionale di Astrofisica in Italy and the Centre National d'Études Spatiales in France.

This research has made us of the NASA/IPAC Extragalactic Database (NED) which is operated by the Jet Propulsion Laboratory, California Institute of Technology, under contract with the National Aeronautics and Space Administration. Part of this work is based on archival data, software, or online services provided by the ASI Science Data Center (ASDC).

Some of the results presented in this paper are based on observations obtained with the HobbyEberly Telescope (HET), a joint project of the University of Texas at Austin, the Pennsylvania State University, Stanford University, Ludwig-Maximilians-Universität München, and GeorgAugust-Universität Göttingen. The HET is named in honor of its principal benefactors, William P. Hobby and Robert E. Eberly. The Marcario Low Resolution Spectrograph (LRS) is named for Mike Marcario of High Lonesome Optics, who fabricated several optics for the instrument but died before its completion. The LRS is a joint project of the Hobby-Eberly Telescope partnership and the Instituto de Astronomía de la Universidad Nacional Autónoma de México.

This work is also partly based on optical spectroscopy performed at the Telescopio Nazionale Galileo (TNG), La Palma, Canary Islands (proposal AOT20/09B). These observations confirm some of the redshifts and classifications found with the HET. We thank the HET and TNG personnel for their assistance during the observing runs.

The data in this paper are based partly on observations obtained at the Hale Telescope, Palomar Observatory, as part of a collaborative agreement between the California Institute of Technology, its divisions Caltech Optical Observatories and the Jet Propulsion Laboratory (operated for NASA), and Cornell University.

Some of the data presented herein were obtained at the W. M. Keck Observatory, which is operated as a scientific partnership among the California Institute of Technology, the University of California, and the National Aeronautics and Space Administration. The Observatory was made 
possible by the generous financial support of the W. M. Keck Foundation.

The authors wish to recognize and acknowledge the very significant cultural role and reverence that the summit of Mauna Kea has always had within the indigenous Hawaiian community. We are most fortunate to have the opportunity to conduct observations from this mountain.

Some of the data in this paper are based on observations made with ESO telescopes at the La Silla Observatory under programs 083.B-0460(B) and 084.B-0711(B).

The National Radio Astronomy Observatory is a facility of the National Science Foundation operated under cooperative agreement by Associated Universities, Inc.

Funding for the Sloan Digital Sky Survey (SDSS) and SDSS-II has been provided by the Alfred P. Sloan Foundation, the Participating Institutions, the National Science Foundation, the U. S. Department of Energy, the National Aeronautics and Space Administration, the Japanese Monbukagakusho, the Max Planck Society, and the Higher Education Funding Council for England. The SDSS Web site is http://www.sdss.org/.

The SDSS is managed by the Astrophysical Research Consortium (ARC) for the Participating Institutions. The Participating Institutions are the American Museum of Natural History, Astrophysical Institute Potsdam, University of Basel, University of Cambridge, Case Western Reserve University, The University of Chicago, Drexel University, Fermilab, the Institute for Advanced Study, the Japan Participation Group, The Johns Hopkins University, the Joint Institute for Nuclear Astrophysics, the Kavli Institute for Particle Astrophysics and Cosmology, the Korean Scientist Group, the Chinese Academy of Sciences (LAMOST), Los Alamos National Laboratory, the Max-Planck-Institute for Astronomy (MPIA), the Max-Planck-Institute for Astrophysics (MPA), New Mexico State University, Ohio State University, University of Pittsburgh, University of Portsmouth, Princeton University, the United States Naval Observatory, and the University of Washington.

Facilities: Fermi LAT.

\section{REFERENCES}

Abdo, A. A., et al. 2009a, ApJ, 700, 597 (LBAS)

-. 2009b, ApJ, 699, 817 (3C 454.3)

-.2009c, ApJ, 699, 31 (NGC 1275)

—. 2009d, ApJ, 707, 55 (M 87)

—. 2009e, ApJ, 707, 1310 (Fermi observations of TeV AGNs)

-. 2009f, ApJS, 183, 46 (BSL) 
—. 2009g, ApJ, 699, 976 (PMN J0948+0022)

-. 2009h, ApJ, 697, 934 (PKS 1454-354)

—. 2009i, ApJ, 707, L142 ( $\gamma$-ray emission from radio-loud narrow-line Seyfert 1s)

—. 2010a, ApJ, submitted; arXiv:1001.4097 ( $\gamma$-ray spectral properties of LBAS blazars)

—. 2010b, ApJ, in preparation (first-year catalog paper)

—. 2010c, ApJ, in preparation (misaligned AGNs)

—. 2010d, ApJ, in preparation (blazar population studies)

-. 2010e, ApJ, in preparation (variability properties of LBAS blazars)

—. 2010f, ApJ, in preparation (unidentified LAT sources)

—. 2010g, ApJ, in preparation ( $\gamma$-ray attenuation by extragalactic background light)

-. 2010h, ApJ, 709, L152 (starburst galaxies)

-. 2010i, ApJ, 708, 1310 (PG 1553+113)

—. 2010j, ApJ, submitted; arXiv:0912.2040 (SEDs of Fermi bright blazars)

Acciari, V. A., et al. 2010, ApJ, 708, L100

Acero, F., et al. 2009, A\&A, in press; arXiv:0911.2709

Adelman-McCarthy, J. K., et al. 2008, ApJS, 175, 297

Aharonian, F., et al. 2009, ApJ, 696, L150

Ajello, M., et al. 2009, ApJ, 699, 603

Antonucci, R. 1993, ARA\&A, 31, 473

Atwood, W. B., et al. 2009, ApJ, 697, 1071

Beasley, A. J., Gordon, D., Peck, A. B., Petrov, L., MacMillan, D. S., Fomalont, E. B., \& Ma, C. 2002, ApJS, 141, 13

Bennett, A. S. 1962, MNRAS, 125, 75

Bird, A. J., et al. 2010, ApJS, 186, 1

Blandford, R. D., \& Rees, M. J. 1978, in BL Lac Objects, ed. A. M. Wolfe, 328-341

Böttcher, M., \& Dermer, C. D. 2002, ApJ, 564, 86 
Browne, I. W. A., et al. 2003, MNRAS, 341, 13

Burgess, A. M., \& Hunstead, R. W. 2006, AJ, 131, 100

Caccianiga, A., Marchã, M. J., Antón, S., Mack, K., \& Neeser, M. J. 2002, MNRAS, 329, 877

Caccianiga, A., \& Marchã, M. J. M. 2004, MNRAS, 348, 937

Campana, R., Massaro, E., Gasparrini, D., Cutini, S., \& Tramacere, A. 2008, MNRAS, 383, 1166

Casandjian, J., \& Grenier, I. A. 2008, A\&A, 489, 849

Cavaliere, A., \& D'Elia, V. 2002, ApJ, 571, 226

Chen, A., Reyes, L. C., \& Ritz, S. 2004, ApJ, 608, 686

Chiang, J., \& Mukherjee, R. 1998, ApJ, 496, 752

Ciprini, S., et al. 2007, in American Institute of Physics Conference Series, Vol. 921, The First GLAST Symposium, ed. S. Ritz, P. Michelson, \& C. A. Meegan, 546-547

Condon, J. J., Cotton, W. D., Greisen, E. W., Yin, Q. F., Perley, R. A., Taylor, G. B., \& Broderick, J. J. 1998, AJ, 115, 1693

Cusumano, G., et al. 2009, A\&A, in press; arXiv:0906.4788

de Ruiter, H. R., Arp, H. C., \& Willis, A. G. 1977, A\&AS, 28, 211

Dermer, C. D. 2007, ApJ, 659, 958

Engelbracht, C. W., Rieke, M. J., Rieke, G. H., Kelly, D. M., \& Achtermann, J. M. 1998, ApJ, 505,639

Falco, E. E., Kochanek, C. S., \& Munoz, J. A. 1998, ApJ, 494, 47

Fichtel, C. E., et al. 1994, ApJS, 94, 551

Fomalont, E. B., Petrov, L., MacMillan, D. S., Gordon, D., \& Ma, C. 2003, AJ, 126, 2562

Förster Schreiber, N. M., Roussel, H., Sauvage, M., \& Charmandaris, V. 2004, A\&A, 419, 501

Fossati, G., Maraschi, L., Celotti, A., Comastri, A., \& Ghisellini, G. 1998, MNRAS, 299, 433

Ghisellini, G., Celotti, A., Fossati, G., Maraschi, L., \& Comastri, A. 1998, MNRAS, 301, 451

Ghisellini, G., Maraschi, L., \& Tavecchio, F. 2009, MNRAS, 396, L105

Ghisellini, G., \& Tavecchio, F. 2008, MNRAS, 387, 1669

Ghisellini, G., et al. 2010, MNRAS, submitted; arXiv:0912.0001 
Giommi, P., Colafrancesco, S., Padovani, P., Gasparrini, D., Cavazzuti, E., \& Cutini, S. 2009, A\&A, 508, 107

Giommi, P., Menna, M. T., \& Padovani, P. 1999, MNRAS, 310, 465

Giommi, P., et al. 2007, A\&A, 468, 97

Hartman, R. C., et al. 1999, ApJS, 123, 79

Healey, S. E., Romani, R. W., Taylor, G. B., Sadler, E. M., Ricci, R., Murphy, T., Ulvestad, J. S., \& Winn, J. N. 2007, ApJS, 171, 61

Healey, S. E., et al. 2008, ApJS, 175, 97

Hill, G. J., Nicklas, H. E., MacQueen, P. J., Tejada, C., Cobos Duenas, F. J., \& Mitsch, W. 1998, in Society of Photo-Optical Instrumentation Engineers (SPIE) Conference Series, ed. S. D'Odorico, Vol. 3355, 375-386

Hinshaw, G., et al. 2007, ApJS, 170, 288

Hofmann, W., \& Fegan, S. 2009, The Astronomer's Telegram, 2293, 1

Inoue, Y., \& Totani, T. 2009, ApJ, 702, 523

Jones, D. H., et al. 2004, MNRAS, 355, 747

—. 2009, MNRAS, 399, 683

Komatsu, E., et al. 2009, ApJS, 180, 330

Kovalev, Y. Y., Petrov, L., Fomalont, E. B., \& Gordon, D. 2007, AJ, 133, 1236

Laing, R. A., Riley, J. M., \& Longair, M. S. 1983, MNRAS, 204, 151

Lamb, R. C., \& Macomb, D. J. 1997, ApJ, 488, 872

Landt, H., Padovani, P., Perlman, E. S., \& Giommi, P. 2004, MNRAS, 351, 83

Lott, B., Ballet, J., Chiang, J., Lonjou, V., \& Funk, S. 2007, in American Institute of Physics Conference Series, Vol. 921, The First GLAST Symposium, ed. S. Ritz, P. Michelson, \& C. A. Meegan, 558-559

Malmquist, K. 1920, Medd. Lunds Astron. Obs., series 2, 22

Maraschi, L., Ghisellini, G., \& Celotti, A. 1992, ApJ, 397, L5

Marchã, M. J. M., Browne, I. W. A., Impey, C. D., \& Smith, P. S. 1996, MNRAS, 281, 425

Mason, K. O., et al. 2000, MNRAS, 311, 456 
Massaro, E., Giommi, P., Leto, C., Marchegiani, P., Maselli, A., Perri, M., Piranomonte, S., \& Sclavi, S. 2009, A\&A, 495, 691

Mattox, J. R., Hartman, R. C., \& Reimer, O. 2001, ApJS, 135, 155

Mattox, J. R., et al. 1996, ApJ, 461, 396

Mauch, T., Murphy, T., Buttery, H. J., Curran, J., Hunstead, R. W., Piestrzynski, B., Robertson, J. G., \& Sadler, E. M. 2003, MNRAS, 342, 1117

Mazin, D., Lindfors, E., Berger, K., Galante, N., Prandini, E., \& Saito, T. 2009, arXiv:0907.0366

Mitton, S., Hazard, C., \& Whelan, J. A. J. 1977, MNRAS, 179, 569

Monet, D. G., et al. 2003, AJ, 125, 984

Mücke, A., \& Pohl, M. 2000, MNRAS, 312, 177

Myers, S. T., et al. 2003, MNRAS, 341, 1

Narumoto, T., \& Totani, T. 2006, ApJ, 643, 81

Nieppola, E., Tornikoski, M., \& Valtaoja, E. 2006, A\&A, 445, 441

Ong, R. A. 2009a, The Astronomer's Telegram, 2301, 1

—. 2009b, The Astronomer's Telegram, 2309, 1

Ong, R. A., \& Fortin, P. 2009, The Astronomer's Telegram, 2272, 1

Padovani, P., \& Giommi, P. 1995, ApJ, 444, 567

Padovani, P., Perlman, E. S., Landt, H., Giommi, P., \& Perri, M. 2003, ApJ, 588, 128

Perley, R. A., Bridle, A. H., \& Willis, A. G. 1984, ApJS, 54, 291

Petrov, L., Kovalev, Y. Y., Fomalont, E. B., \& Gordon, D. 2005, AJ, 129, 1163

-. 2006, AJ, 131, 1872

-. 2008, AJ, 136, 580

Piranomonte, S., \& D'Elia, V. 2010, A\&A, in preparation

Pittori, C., et al. 2009, A\&A, 506, 1563

Plotkin, R. M., et al. 2010, AJ, 139, 390

Raue, M., Behera, B., Charbonnier, A., Giebels, B., Hauser, M., Kosack, K., Punch, M., \& Zechlin, H. 2009, The Astronomer's Telegram, 2350, 1 
Shaw, M. S., Romani, R. W., Healey, S. E., Cotter, G., Michelson, P. F., \& Readhead, A. C. S. 2010, ApJ, in preparation

Sowards-Emmerd, D., Romani, R. W., \& Michelson, P. F. 2003, ApJ, 590, 109

Spinrad, H., Marr, J., Aguilar, L., \& Djorgovski, S. 1985, PASP, 97, 932

Starck, J.-L., \& Pierre, M. 1998, A\&AS, 128, 397

Stecker, F. W., \& Salamon, M. H. 1996, ApJ, 464, 600

Stocke, J. T., Morris, S. L., Gioia, I. M., Maccacaro, T., Schild, R., Wolter, A., Fleming, T. A., \& Henry, J. P. 1991, ApJS, 76, 813

Sutherland, W., \& Saunders, W. 1992, MNRAS, 259, 413

Tavani, M., et al. 2008, Nuclear Instruments and Methods in Physics Research A, 588, 52

Thompson, D. J., et al. 1993, ApJS, 86, 629

Ubertini, P., Sguera, V., Stephen, J. B., Bassani, L., Bazzano, A., \& Bird, A. J. 2009, ApJ, 706, L7

Urry, C. M., \& Padovani, P. 1995, PASP, 107, 803

Vermeulen, R. C., Taylor, G. B., Readhead, A. C. S., \& Browne, I. W. A. 1996, AJ, 111, 1013

Véron-Cetty, M., \& Véron, P. 1993, A\&AS, 100, 521

-. 2006, A\&A, 455, 773

Wills, K. A., Morganti, R., Tadhunter, C. N., Robinson, T. G., \& Villar-Martin, M. 2004, MNRAS, 347,771

Wills, K. A., Pedlar, A., Muxlow, T. W. B., \& Stevens, I. R. 1999, MNRAS, 305, 680 


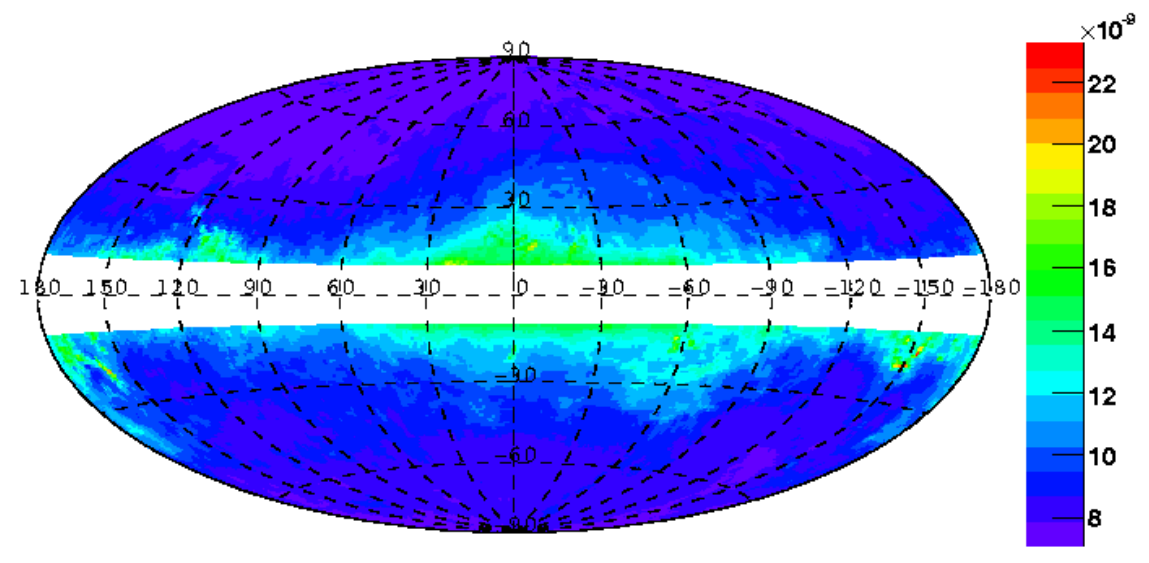

Fig. 1.- Point-source flux limit in units of photons $\mathrm{cm}^{-2} \mathrm{~s}^{-1}$ for $E>100 \mathrm{MeV}$ and photon spectral index $\Gamma=2.2$ as a function of sky location (in Galactic coordinates).

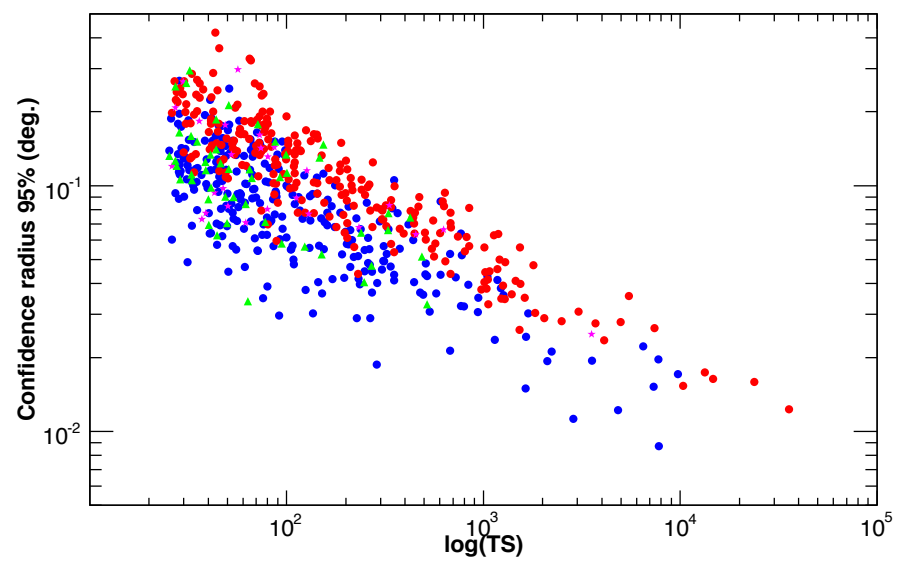

Fig. 2.- $95 \%$ error radius vs. TS for the 1LAC clean sample. Red circles: FSRQs, blue circles: BL Lacs, magenta stars: radio galaxies, green triangles: AGNs of unknown type. 


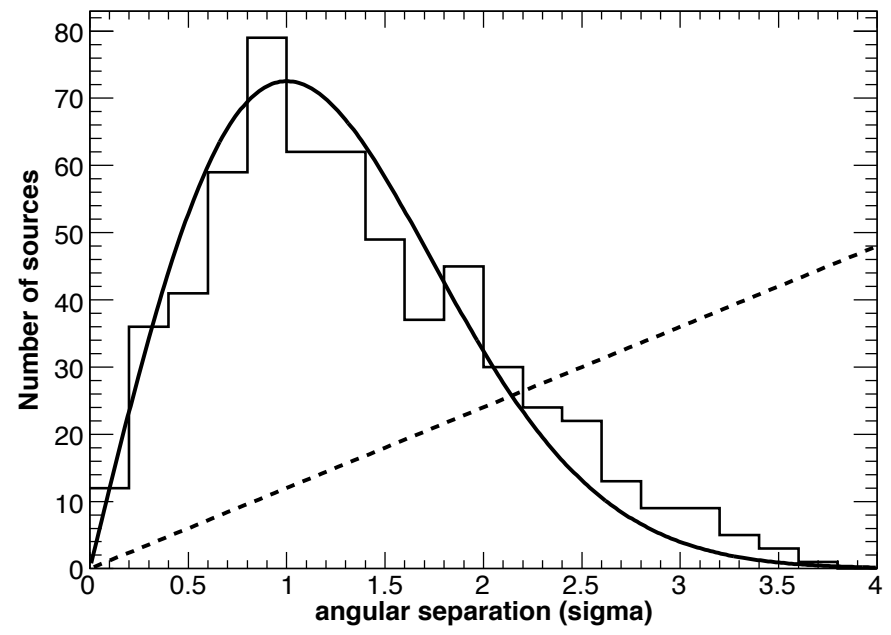

Fig. 3.- Normalized angular separation between the Fermi-LAT source position and that of the AGN counterpart. The solid curve corresponds to the expected distribution $\left(\chi^{2}\right.$ with two degrees of freedom) for real associations, the dashed one for purely accidental associations. 


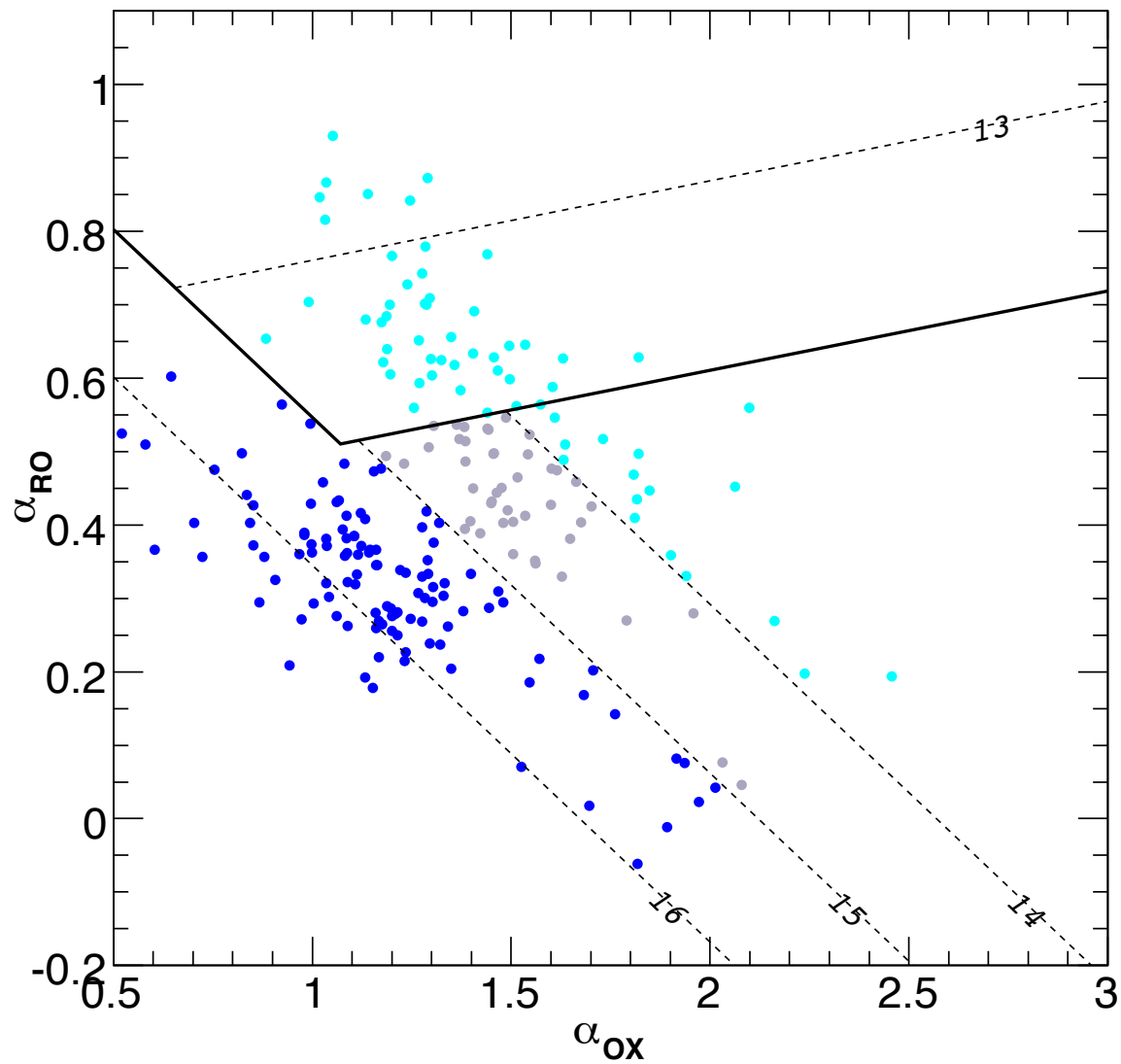

Fig. 4.- Loci of the BL Lacs in the clean sample in the $\left(\alpha_{\mathrm{ox}}, \alpha_{\mathrm{ro}}\right)$ plane (cyan: LSP-BL Lacs, gray: ISP-BL Lacs, blue: HSP-BL Lacs). The $\{X<0\} \cap\{Y<0.3\}$ region (see Section 3.3.2) is delineated in solid black. The dashed lines correspond to $\log \left(\nu_{\text {peak }}^{\mathrm{S}}[\mathrm{Hz}]\right)=13,14,15$, and 16 . 


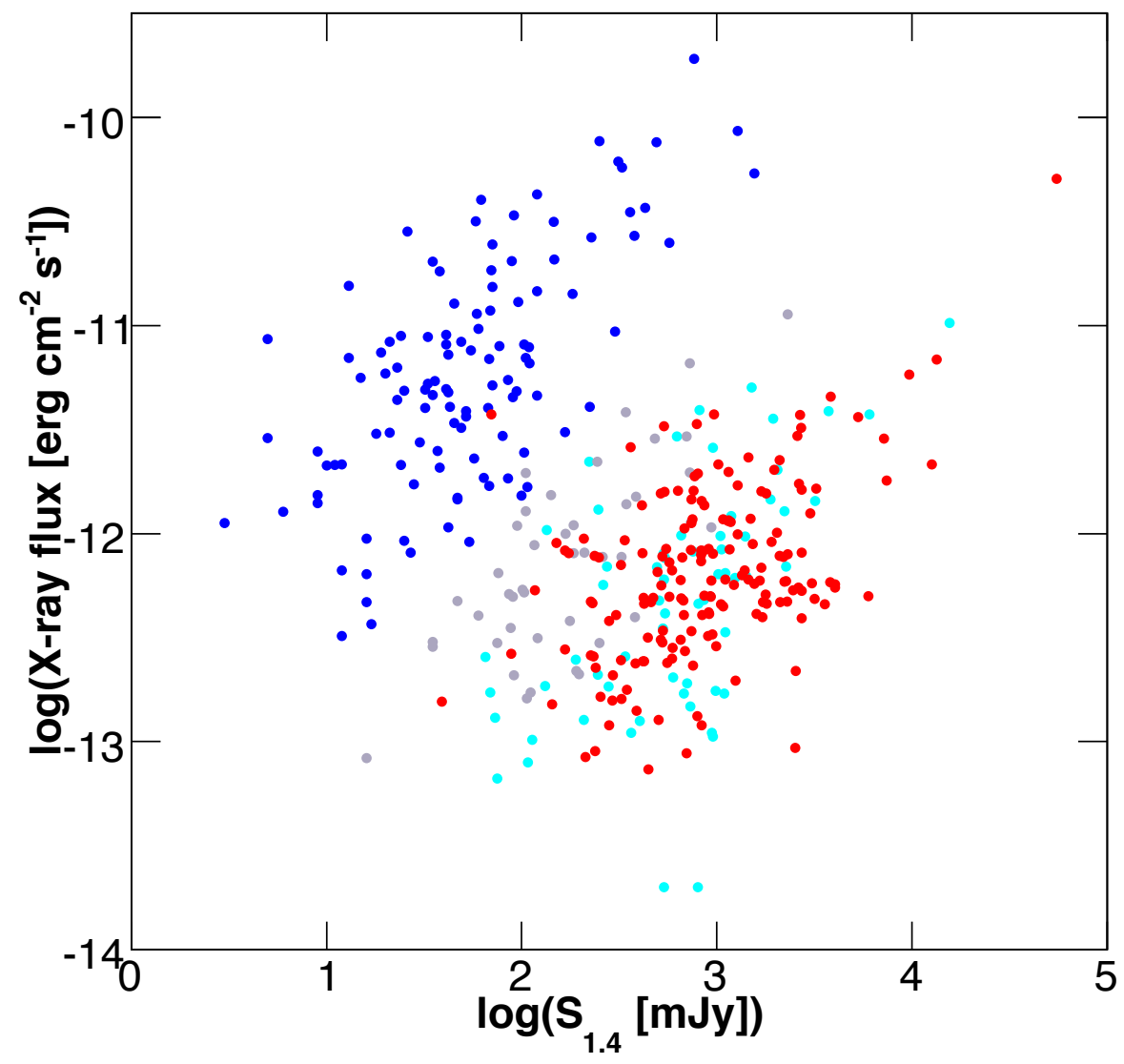

Fig. 5.- Soft X-ray (0.1-2.4 keV) flux vs. radio flux density at $1.4 \mathrm{GHz}$ for the clean sample (red: FSRQs, cyan: LSP-BL Lacs, gray: ISP-BL Lacs, blue: HSP-BL Lacs). 


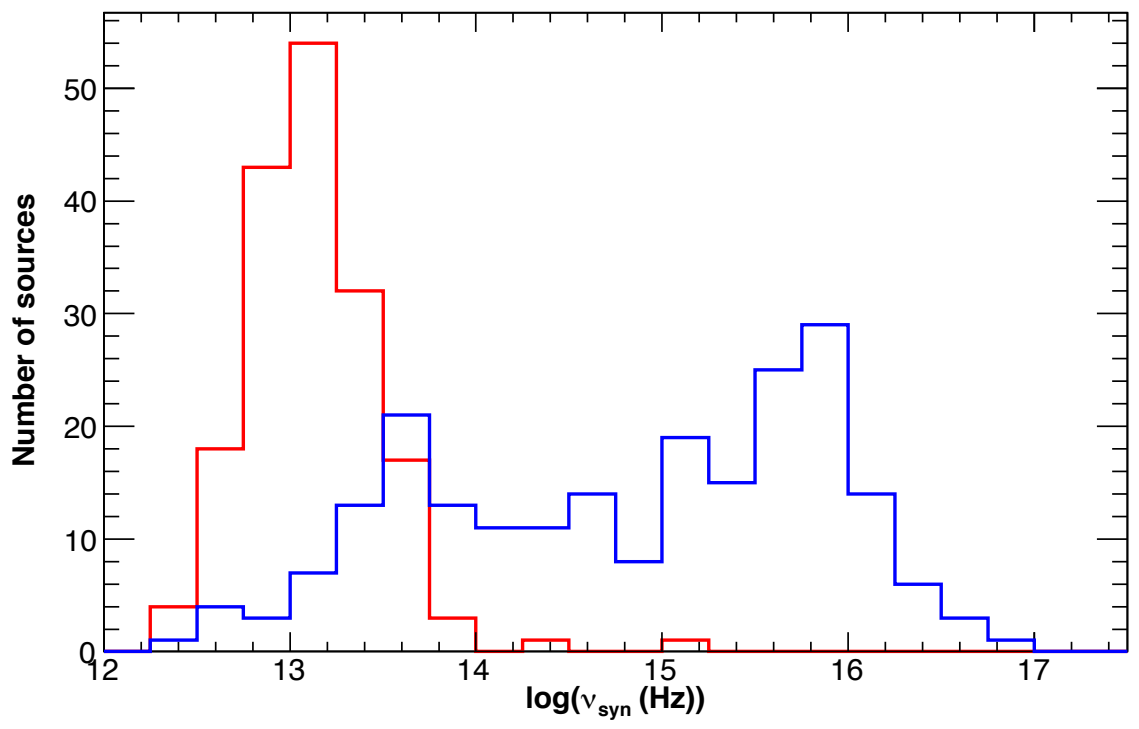

Fig. 6.- Distributions of $\nu_{\text {peak }}^{\mathrm{S}}$ for FSRQs (red) and BL Lacs (blue) in the clean sample.

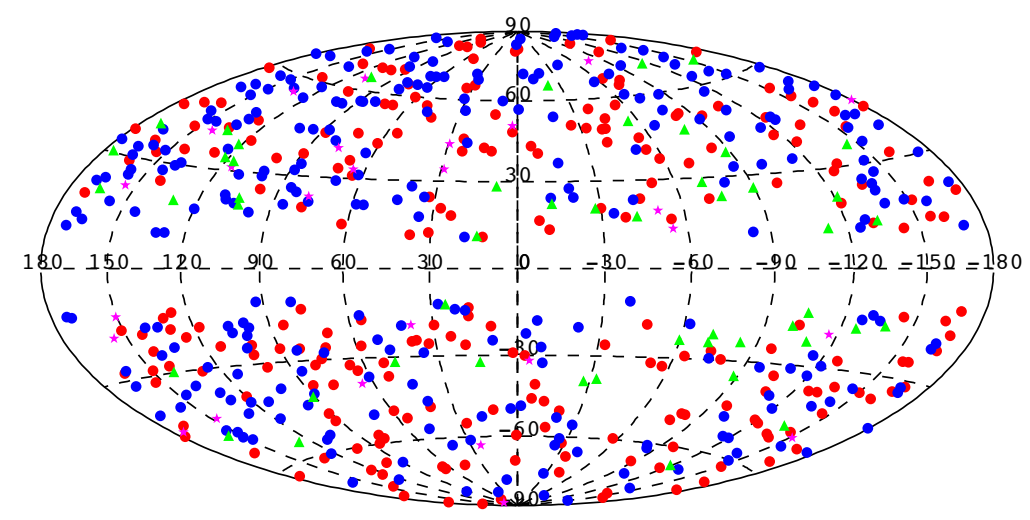

Fig. 7.- Locations of the sources in the clean sample. Red: FSRQs, blue: BL Lacs, magenta: radio galaxies, green: AGNs of unknown type. 

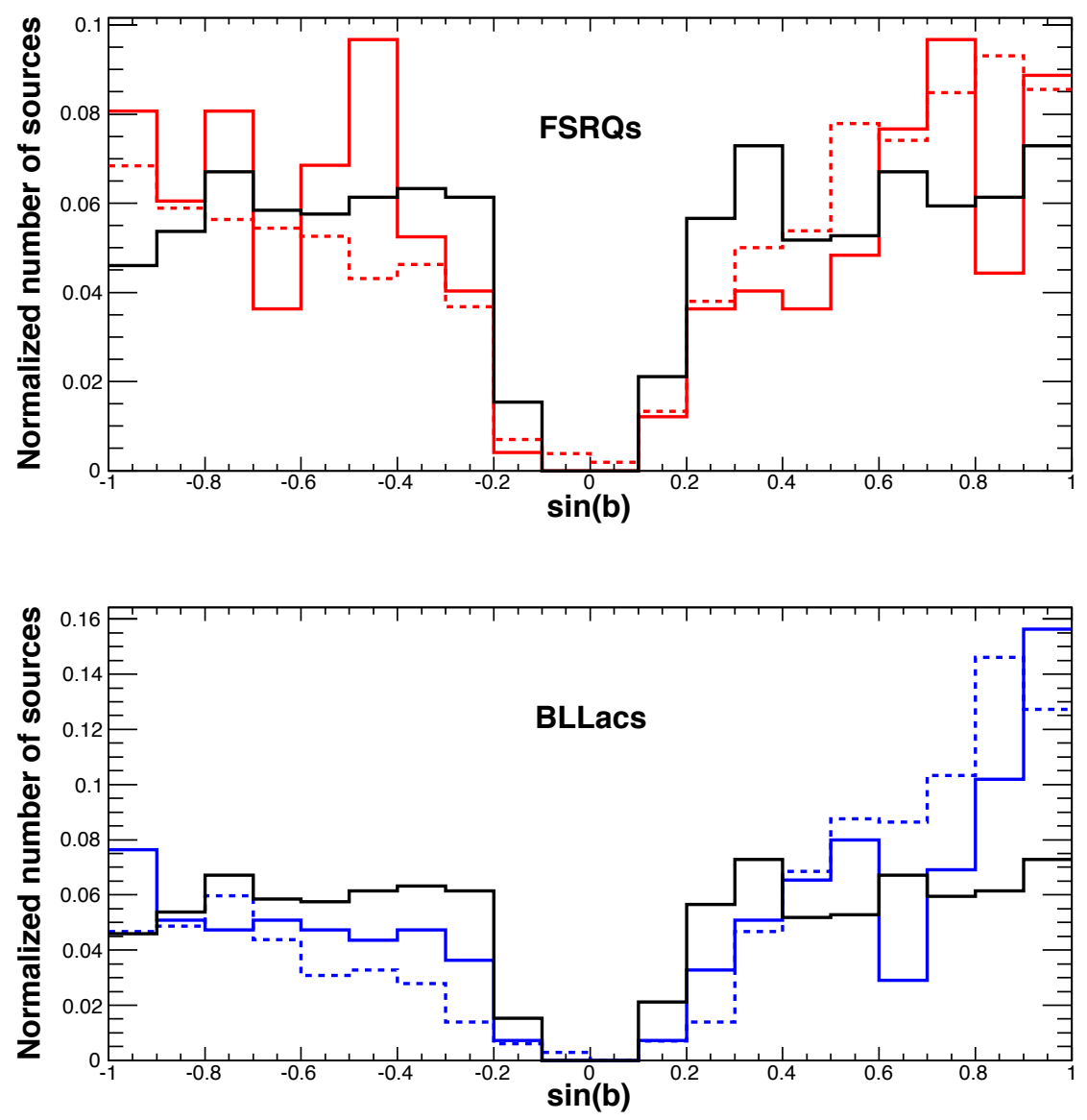

Fig. 8.- Galactic latitude distributions of FSRQs (top) and BL Lacs (bottom) in the clean sample (solid red). The corresponding distributions for the Roma-BZCAT blazars (dashed red) and the 1FGL sources with $|b|>10^{\circ}$ (solid black) are shown for comparison. 

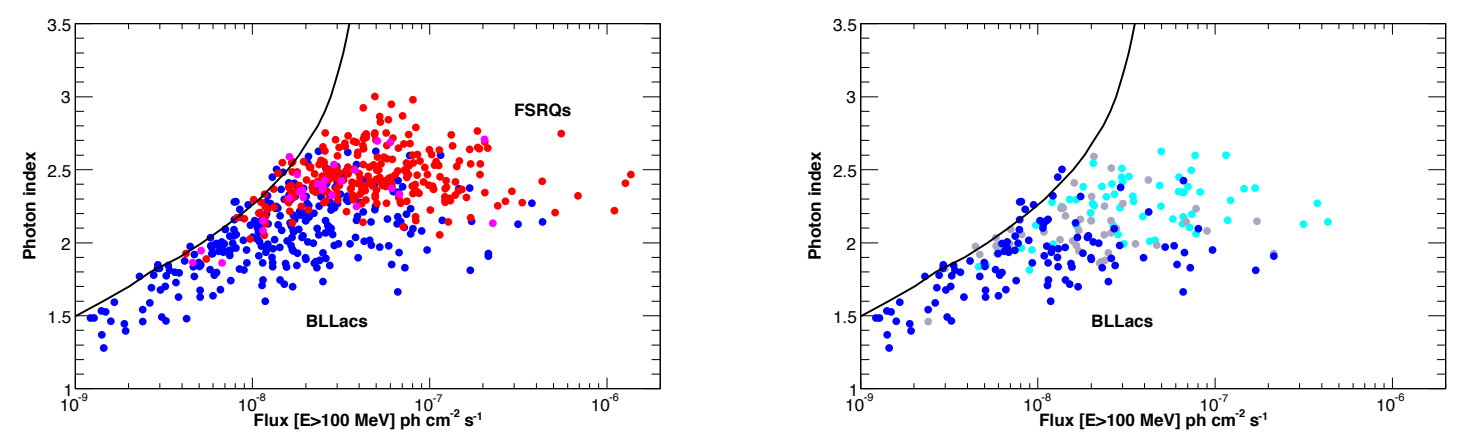

Fig. 9.- Left: Flux $(E>100 \mathrm{MeV})$ vs. photon spectral index for the sources in the clean sample. The solid curves represents the $T S=25$ flux limit estimated for a Galactic position $(l, b)=\left(40^{\circ}\right.$, $\left.40^{\circ}\right)$. Right: the same for the different BL Lac subclasses. Cyan: LSPs, gray: ISPs, blue: HSPs.

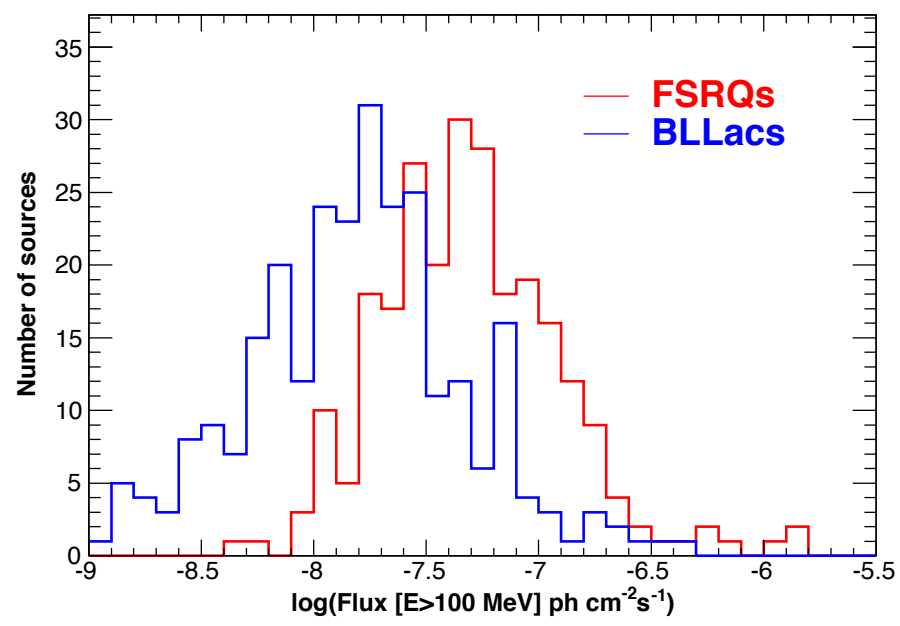

Fig. 10.- Flux $(E>100 \mathrm{MeV})$ distributions for the FSRQs (red) and BL Lacs (blue) in the clean sample. 

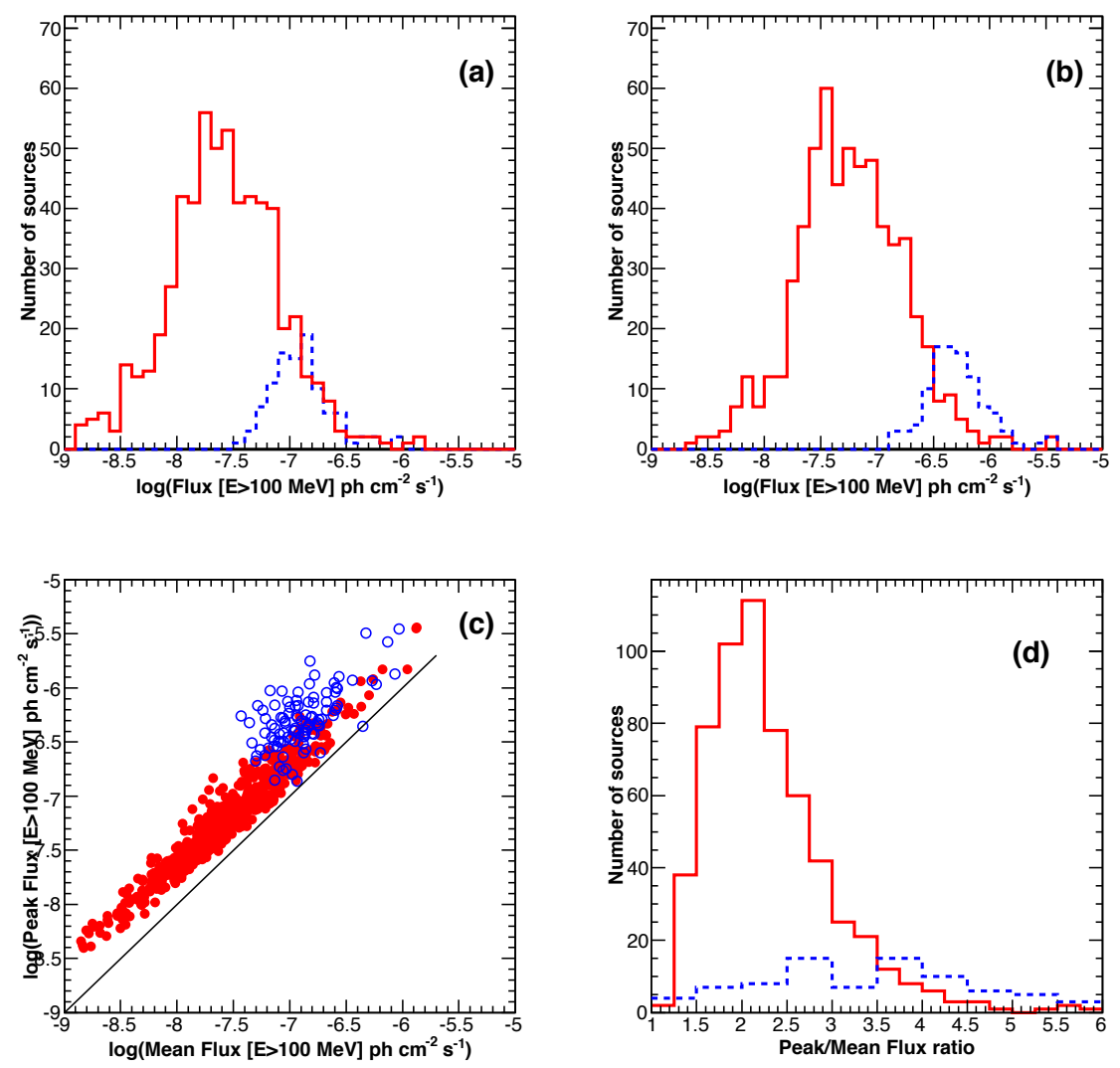

Fig. 11.- (a) Comparison of mean flux distributions for blazars detected by the Fermi-LAT (solid) and by EGRET (dashed). (b) Same as (a) but for the peak flux (i.e., maximum flux in a 15-day viewing period for EGRET, maximum monthly flux for the LAT) distributions. (c) Peak flux vs. mean flux for 1LAC AGNs (filled circles) and for EGRET AGNs (open circles). (d) Same as (a), but for the peak flux/mean flux ratio. 

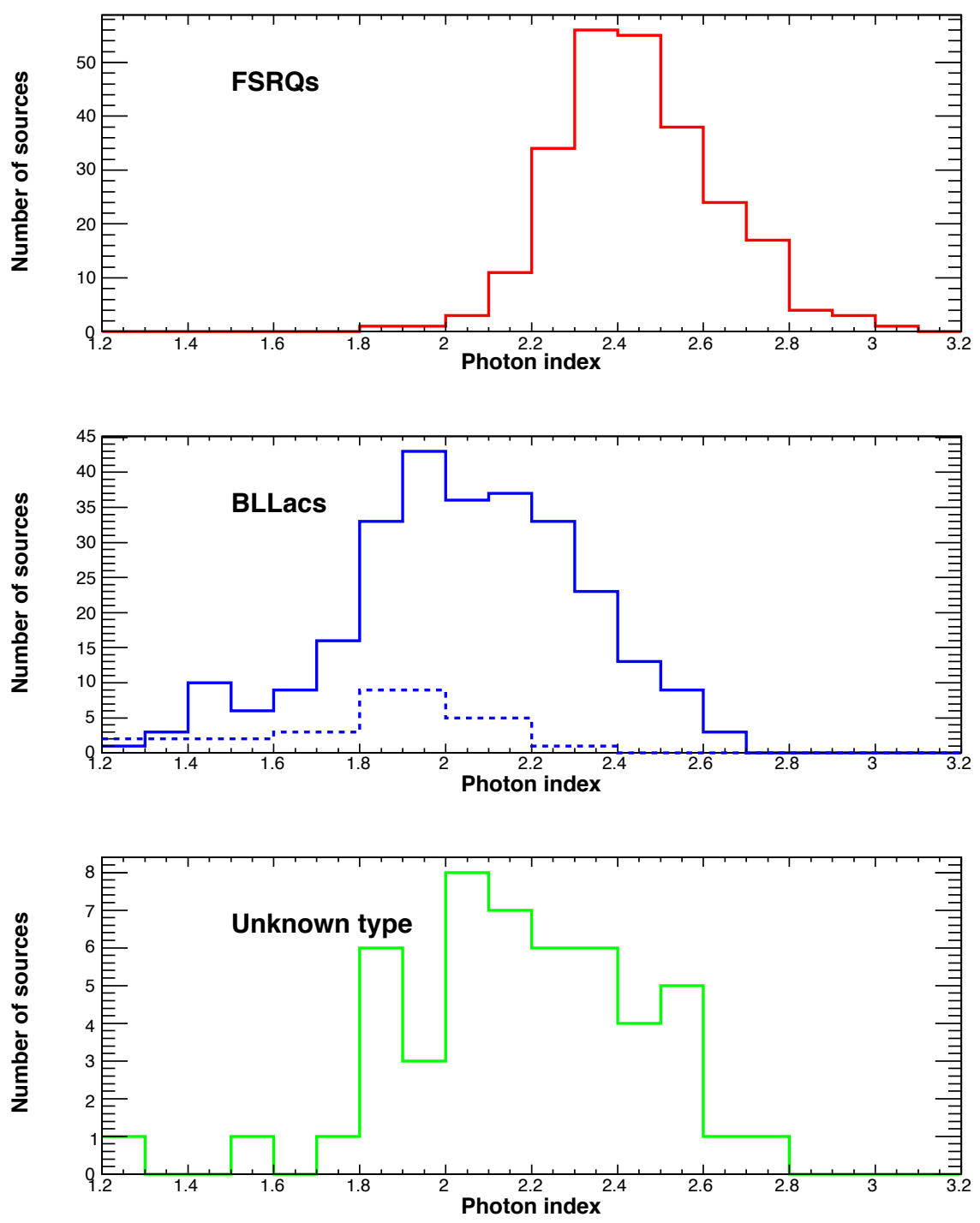

Fig. 12.- Photon spectral index distributions for the FSRQs (top), BL Lacs (middle), and AGNs of unknown type (bottom) in the clean sample. For BL Lacs, the dashed histogram corresponds to the TeV-detected sources. 


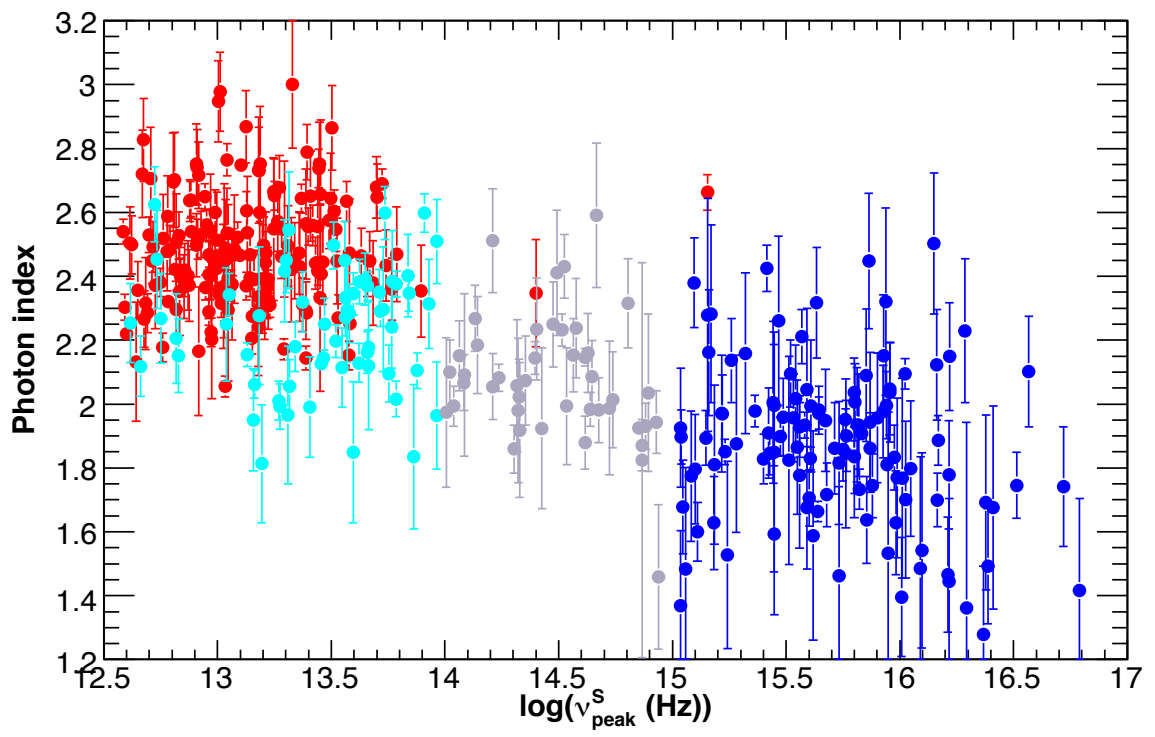

Fig. 13. - Photon spectral index vs. peak frequency of the synchrotron component of the SED for FSRQs (red) and BL Lacs (cyan: LSPs, gray: ISPs, blue: HSPs) in the clean sample. 

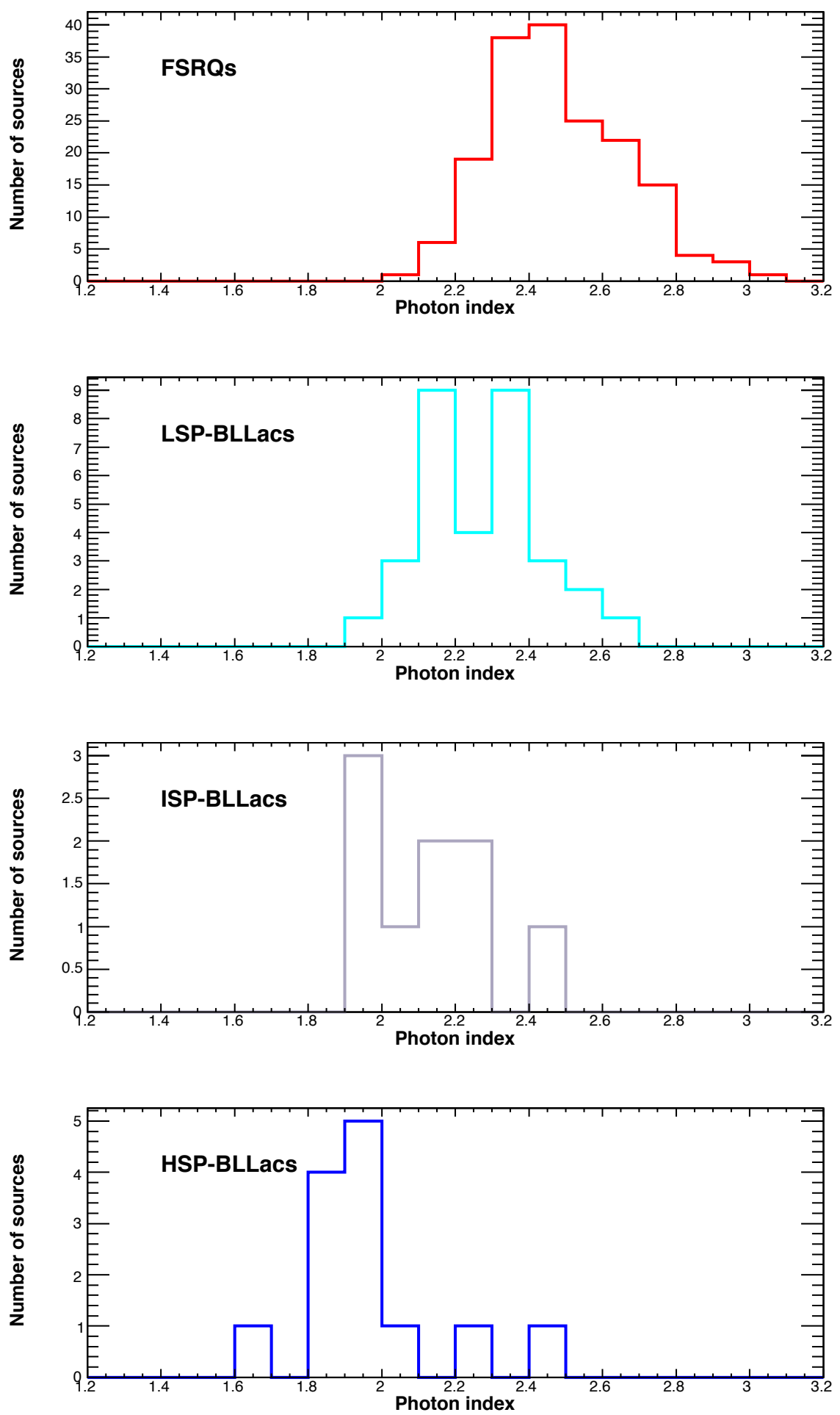

Fig. 14.- Photon spectral index distributions for the different blazar classes for sources in the clean sample with $F[E>100 \mathrm{MeV}]>3 \times 10^{-8}$ photons $\mathrm{cm}^{-2} \mathrm{~s}^{-1}$. 


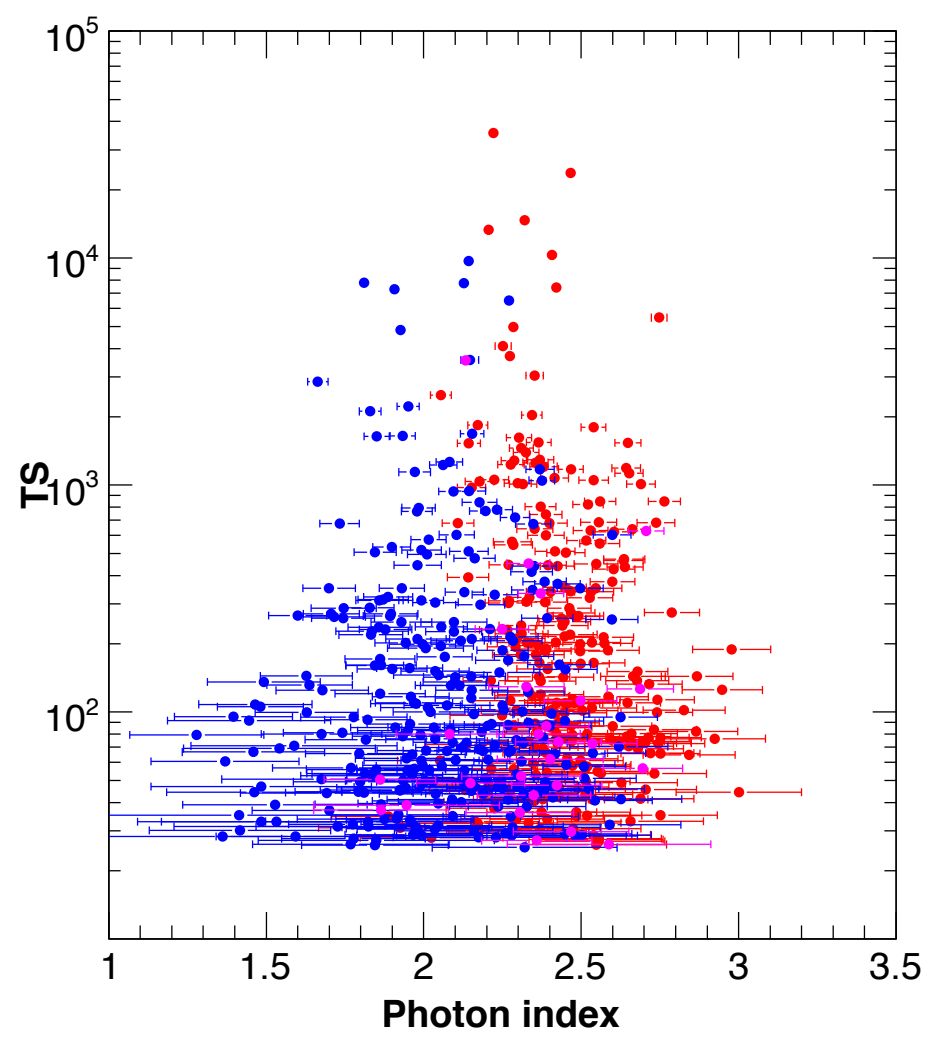

Fig. 15. - TS vs. photon spectral index for FSRQs (red) and BL Lacs (blue) in the clean sample. 


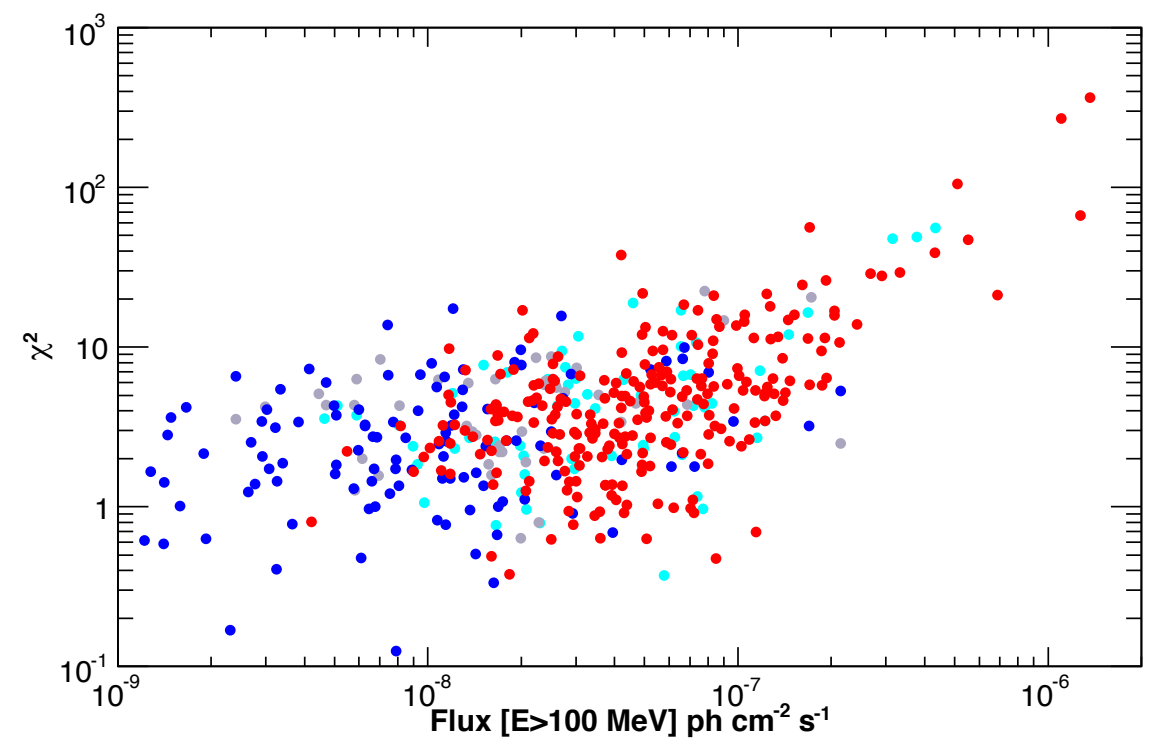

Fig. 16. - Spectral curvature index vs. flux for FSRQs (red) and BL Lacs (cyan: LSPs, gray: ISPs, blue: HSPs) in the clean sample. 


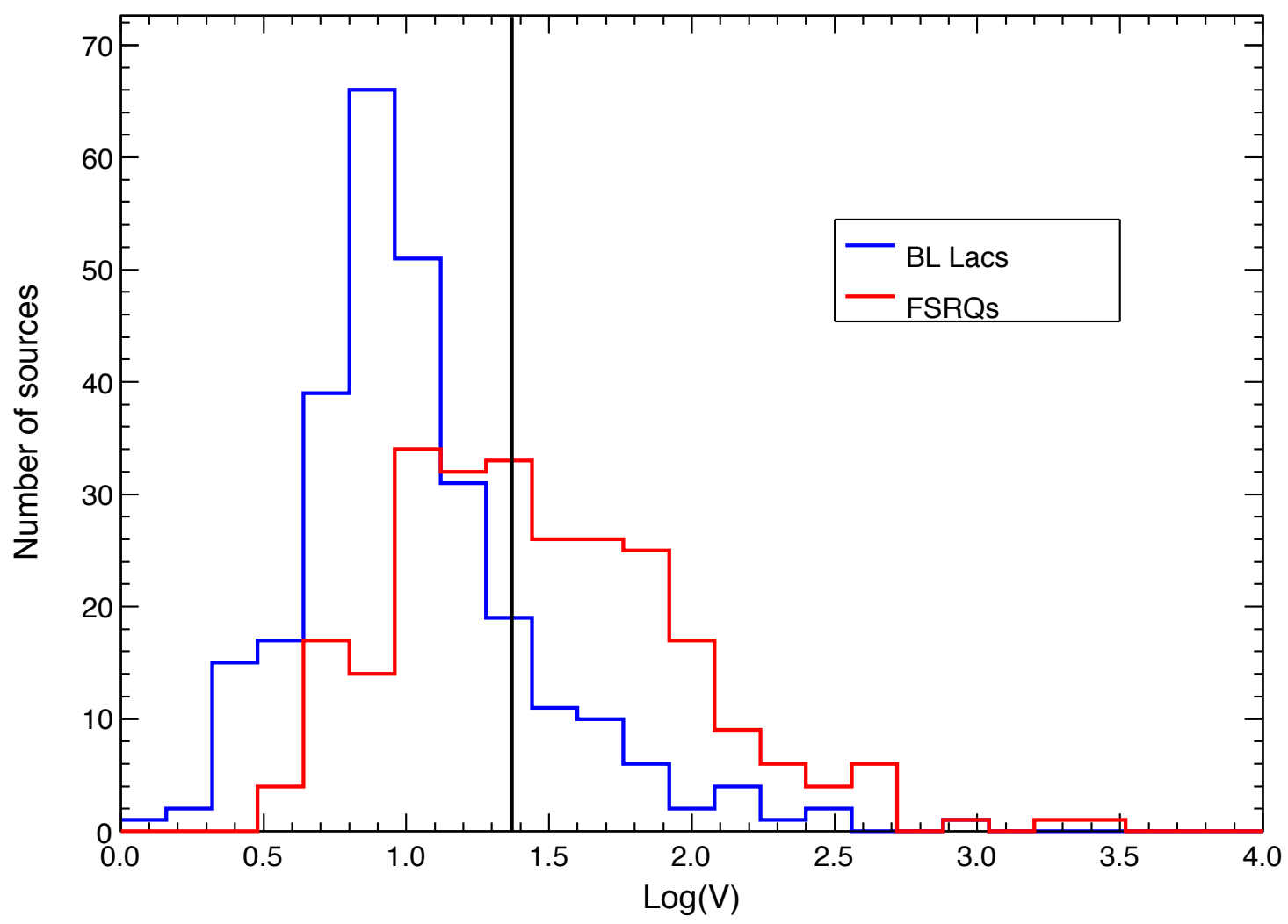

Fig. 17.- Distribution of the variability index for all BL Lacs and FSRQs in the clean sample. The vertical line is drawn at $V=23.21$, which corresponds to a $99 \%$ probability that a source is variable. 


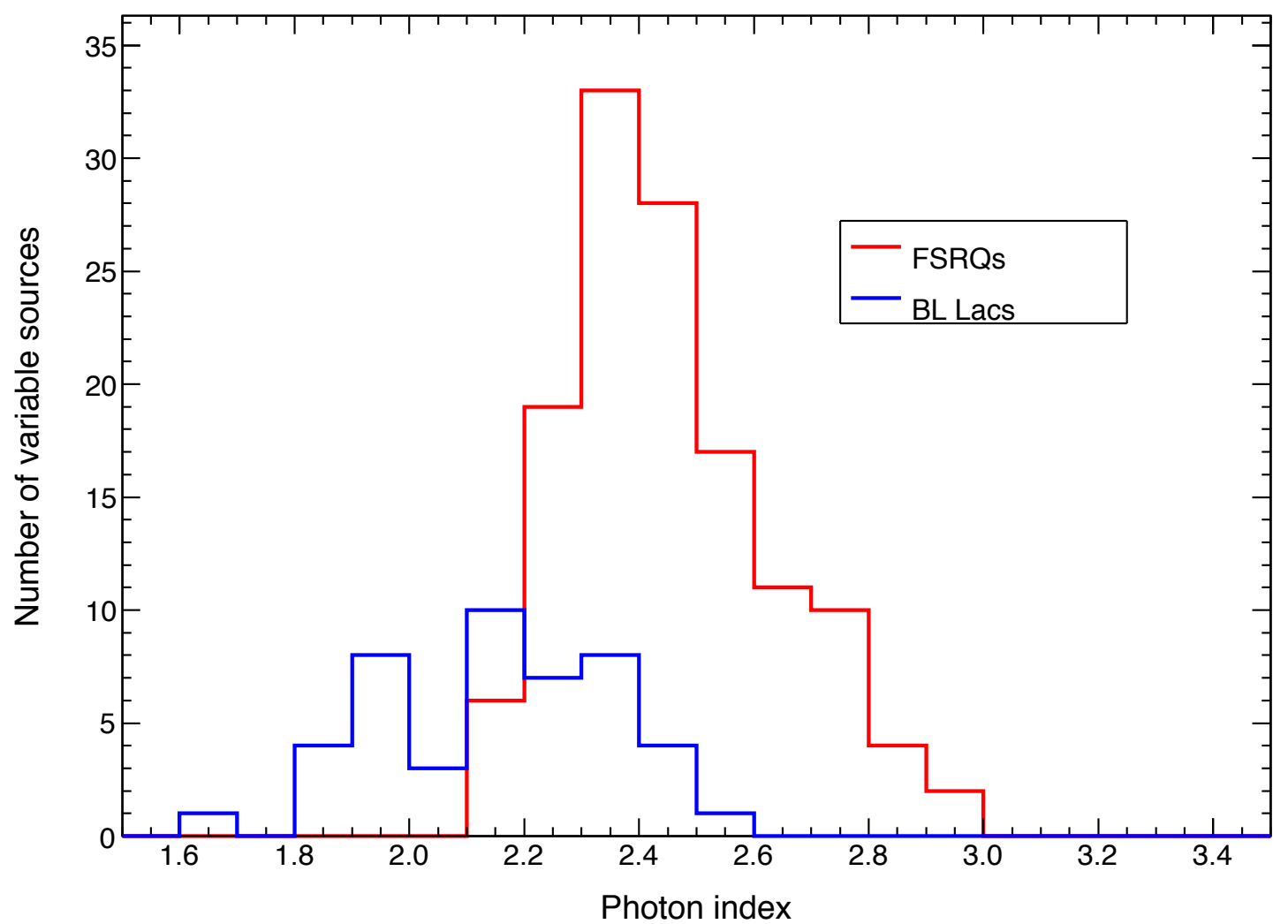

Fig. 18.- Distribution of the photon spectral index for the variable BL Lacs and FSRQs in the clean sample. 


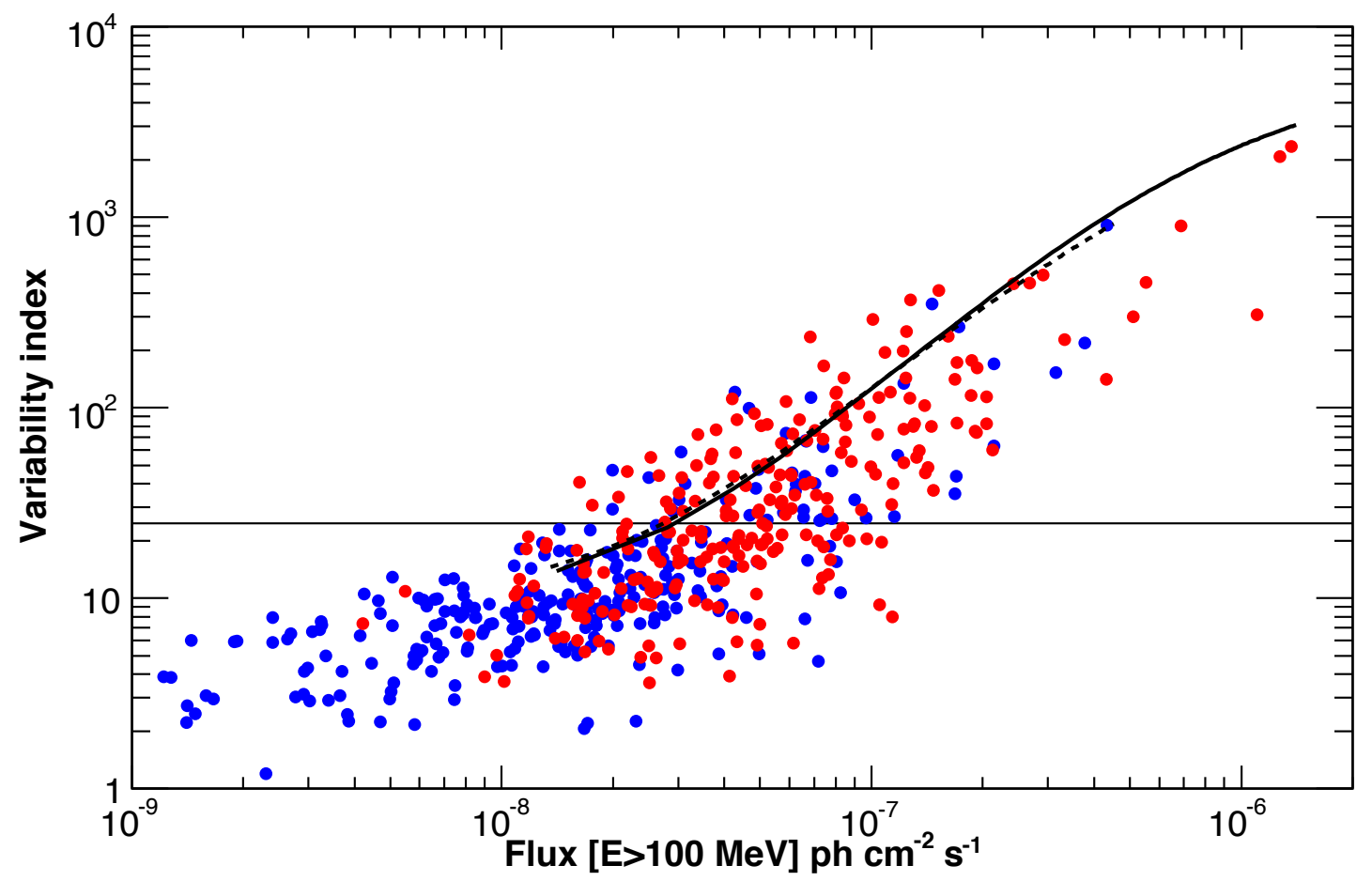

Fig. 19.- Variability index versus flux for FSRQs (red) and BL Lacs (blue) in the clean sample. The curves display the evolution of the variability index for the FSRQ 3C 454.3 (solid) and the BL Lac object AO $0235+164$ (dashed) that would be observed for the same temporal variation but lower mean fluxes. 

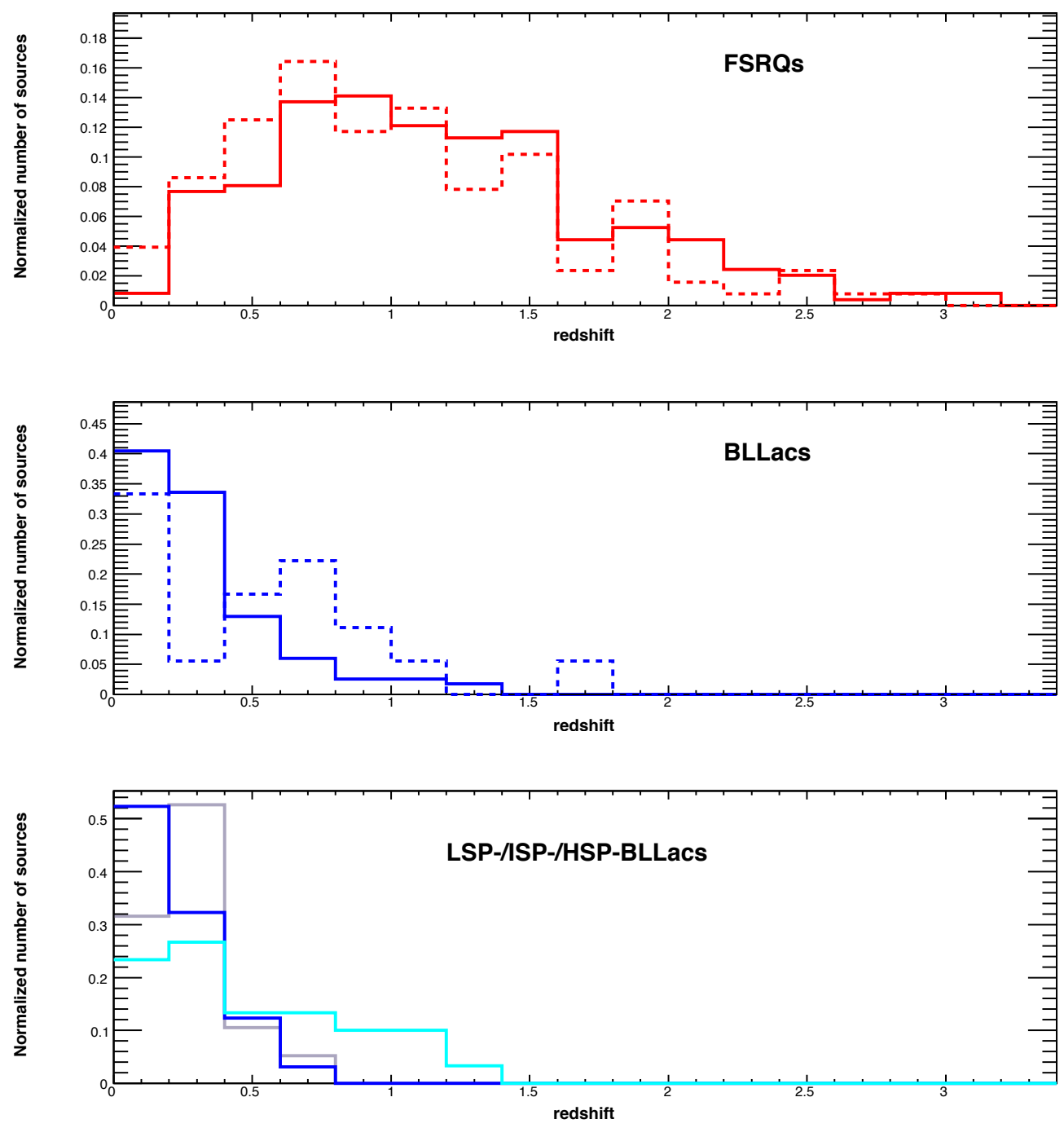

Fig. 20.- Top: Normalized redshift distribution for the FSRQs in the clean sample (solid histogram). The redshift distribution for FSRQs in the WMAP catalog is also shown for comparison (dashed histogram). Middle: The same for BL Lacs. Bottom: Redshift distributions for LSPBL Lacs (cyan), ISP-BL Lacs (gray), HSP-BL Lacs (blue) in the clean sample. 


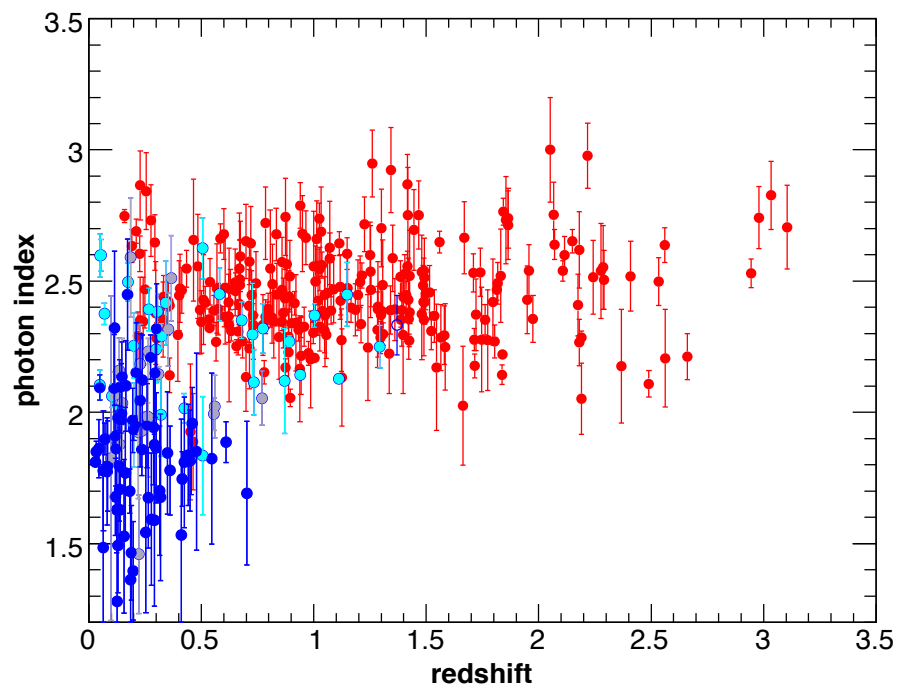

Fig. 21. - Photon spectral index vs. redshift for the FSRQs (red) and BL Lacs (cyan: LSPs, gray: ISPs, blue: HSPs) in the clean sample. 

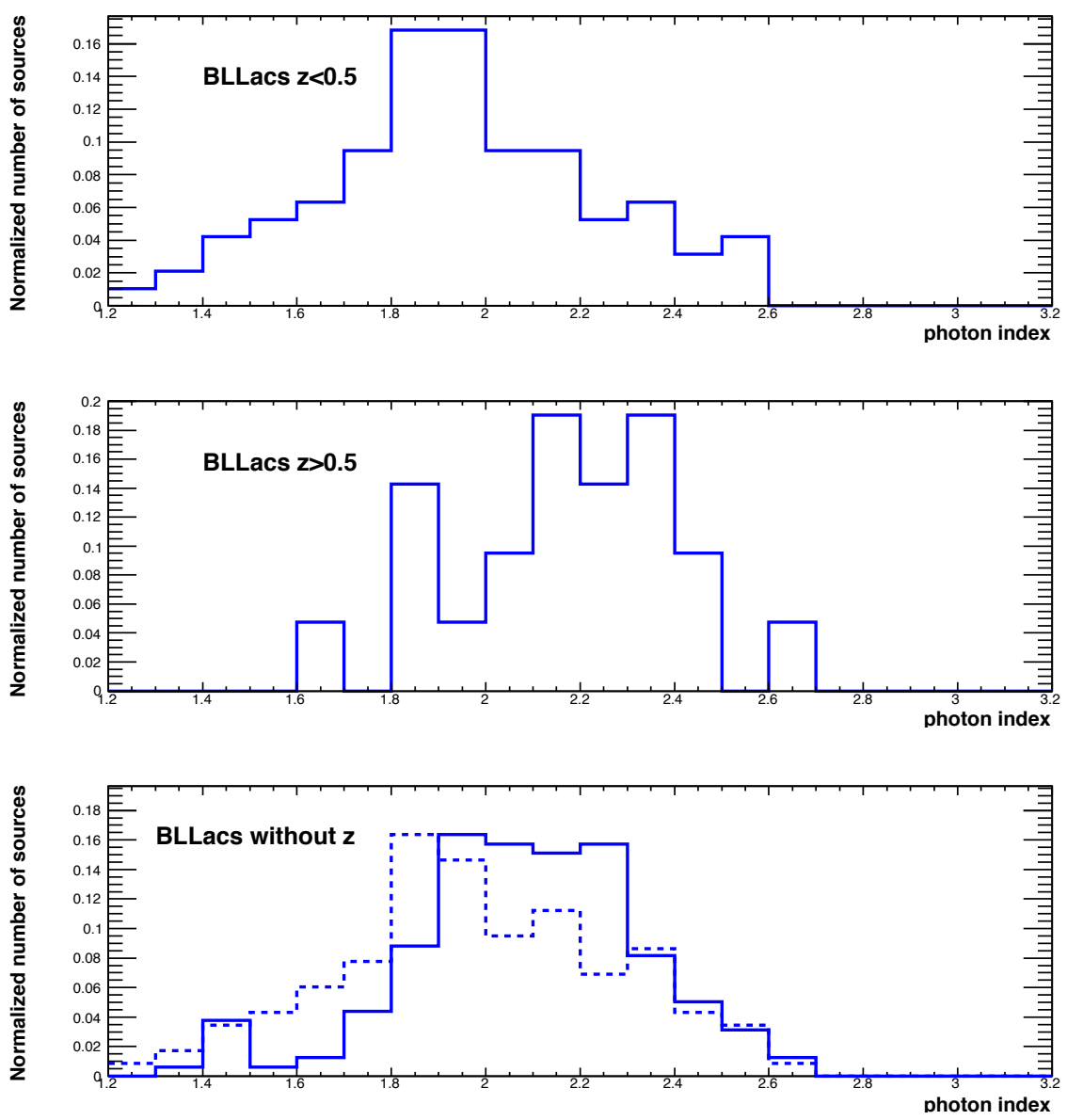

Fig. 22.- Photon spectral index distributions for BL Lacs in the clean sample with $z<0.5$ (top), with $z>0.5$ (middle), and with unknown redshifts (bottom, solid histogram). The total distribution for BL Lacs with known redshifts is shown for comparison as a dashed histogram in the bottom panel. 


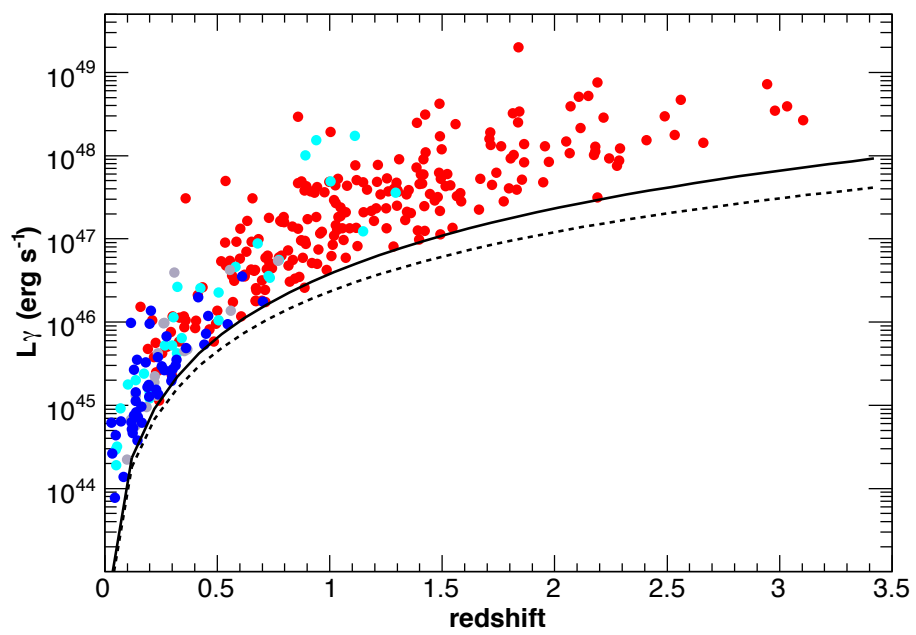

Fig. 23. - $\gamma$-ray luminosity vs. redshift for the different AGN classes (red: FSRQs, cyan: LSPBL Lacs, gray: ISP-BL Lacs, blue: HSP-BL Lacs, magenta: radio galaxies) in the clean sample. The curves correspond to approximate experimental limits calculated for a photon spectral index $\Gamma=2.2$ (solid) and $\Gamma=1.8$ (dashed).

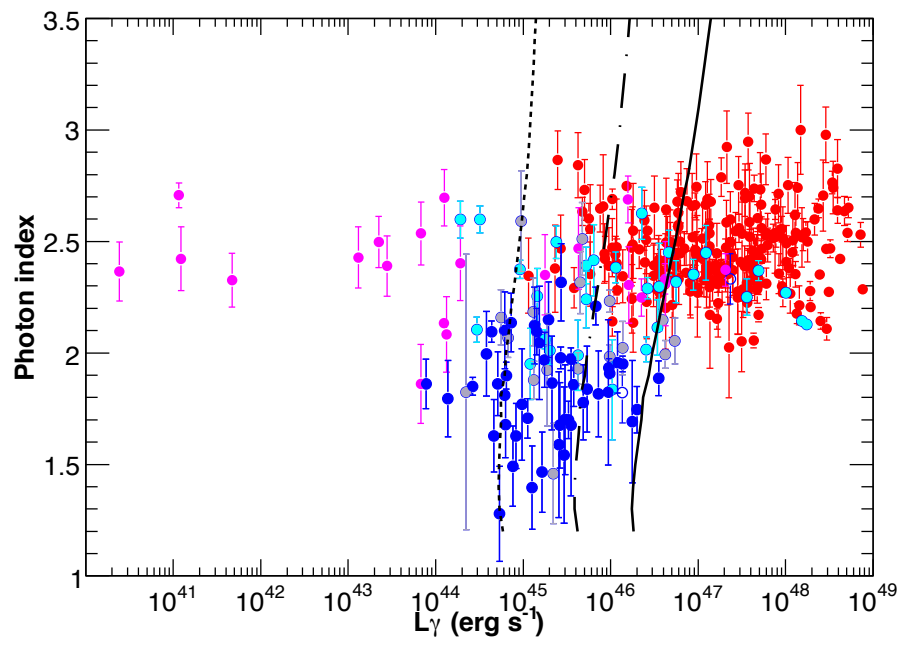

Fig. 24.- Photon spectral index vs. $\gamma$-ray luminosity for the different AGN classes (red: FSRQs, cyan: LSP-BL Lacs, gray: ISP-BL Lacs, blue: HSP-BL Lacs, magenta: radio galaxies) in the clean sample. The curves represent approximate instrumental limits for $z=0.2$ (dashed), $z=0.5$ (dot-dashed), and $z=1$ (solid). 


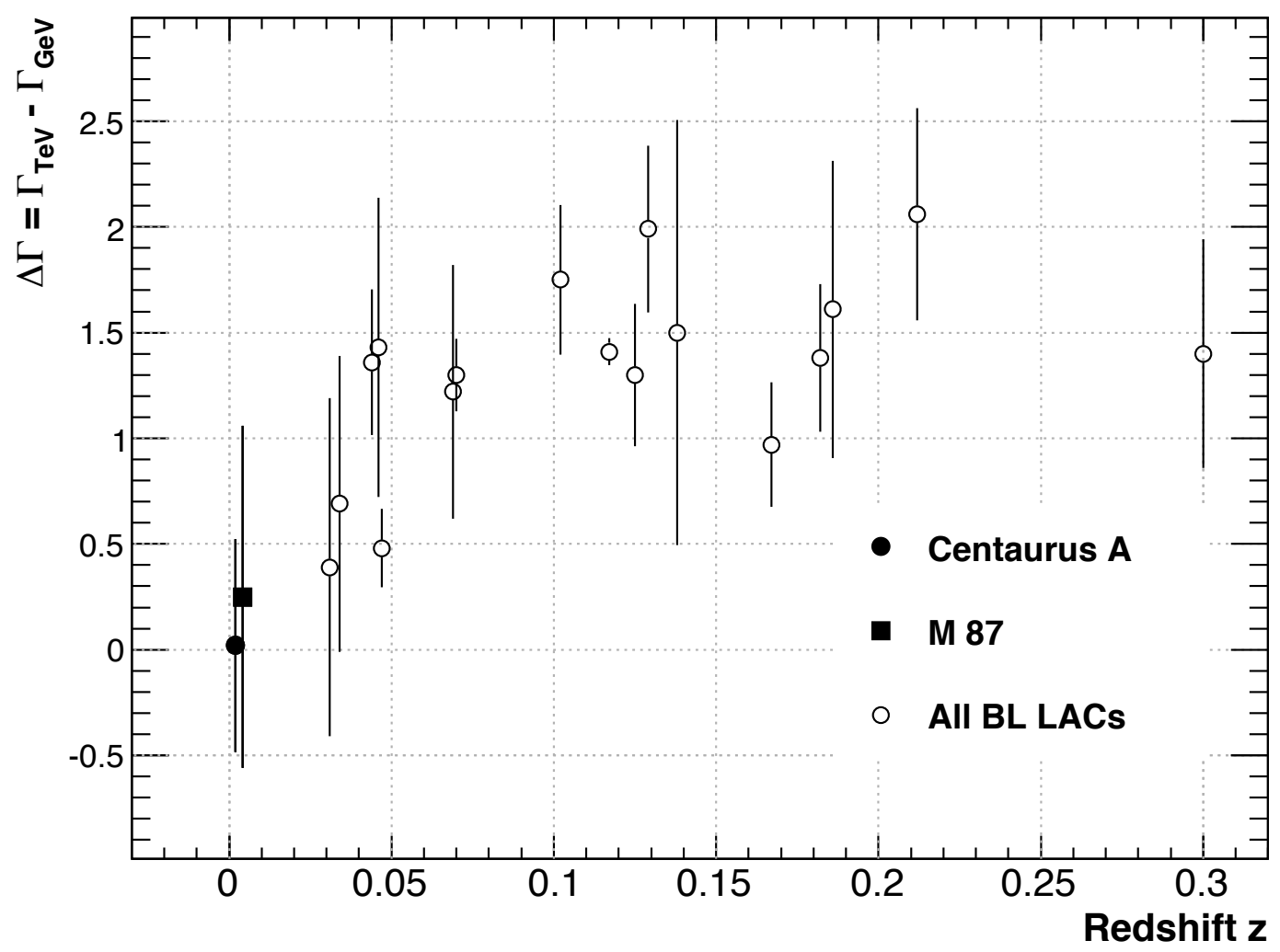

Fig. 25. $-\mathrm{GeV} / \mathrm{TeV}$ spectral break $\Delta \Gamma \equiv \Gamma_{\mathrm{TeV}}-\Gamma_{\mathrm{GeV}}$ vs. redshift. Only sources with well measured redshifts and $\mathrm{TeV}$ photon spectral indices are shown. The TeV measurements come from the references given in Abdo et al. (2009e); new measurements for PKS 2005-489 (Acero et al. 2009) and S5 0716+714 (Mazin et al. 2009) are used. 3C 279, an FSRQ whose TeV spectrum was measured during an extreme flaring episode, is also excluded. 

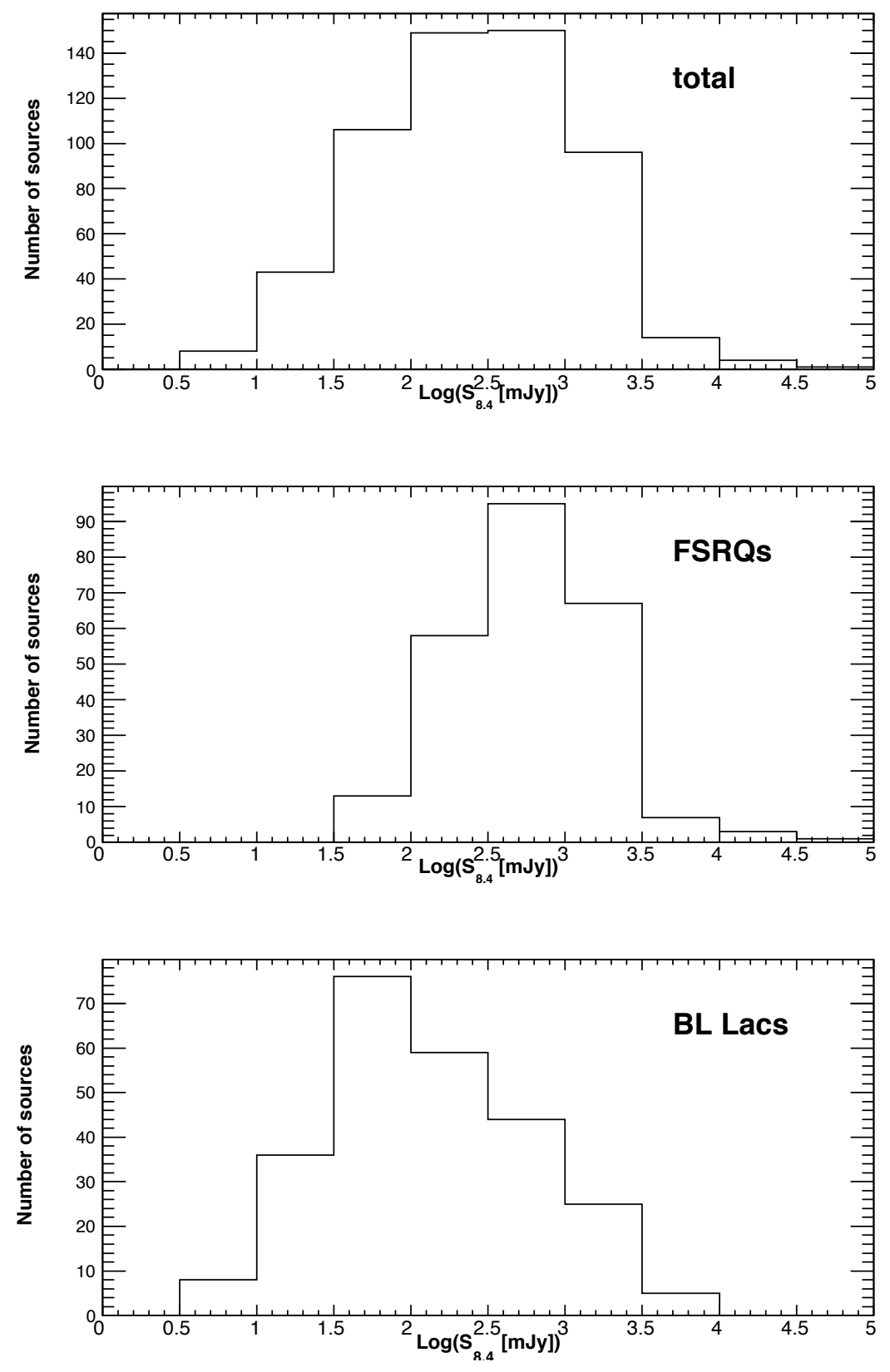

Fig. 26.- Distributions of radio flux densities at $8.4 \mathrm{GHz}$ for all sources in the clean sample (top), FSRQs (middle), and BL Lacs (bottom). 

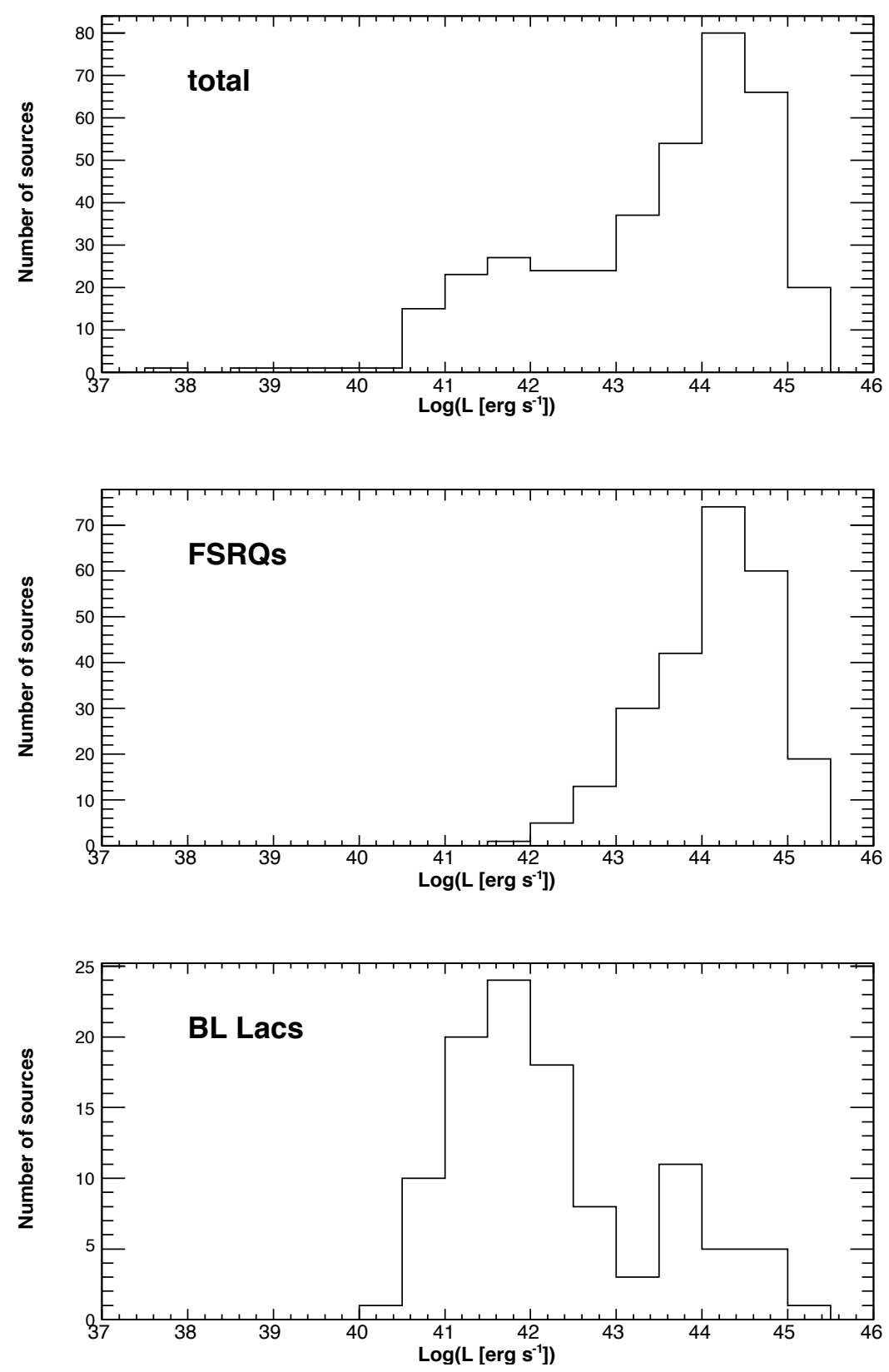

Fig. 27.- Distributions of radio luminosities at $8.4 \mathrm{GHz}$ for all sources in the clean sample (top), FSRQs (middle), and BL Lacs (bottom). 


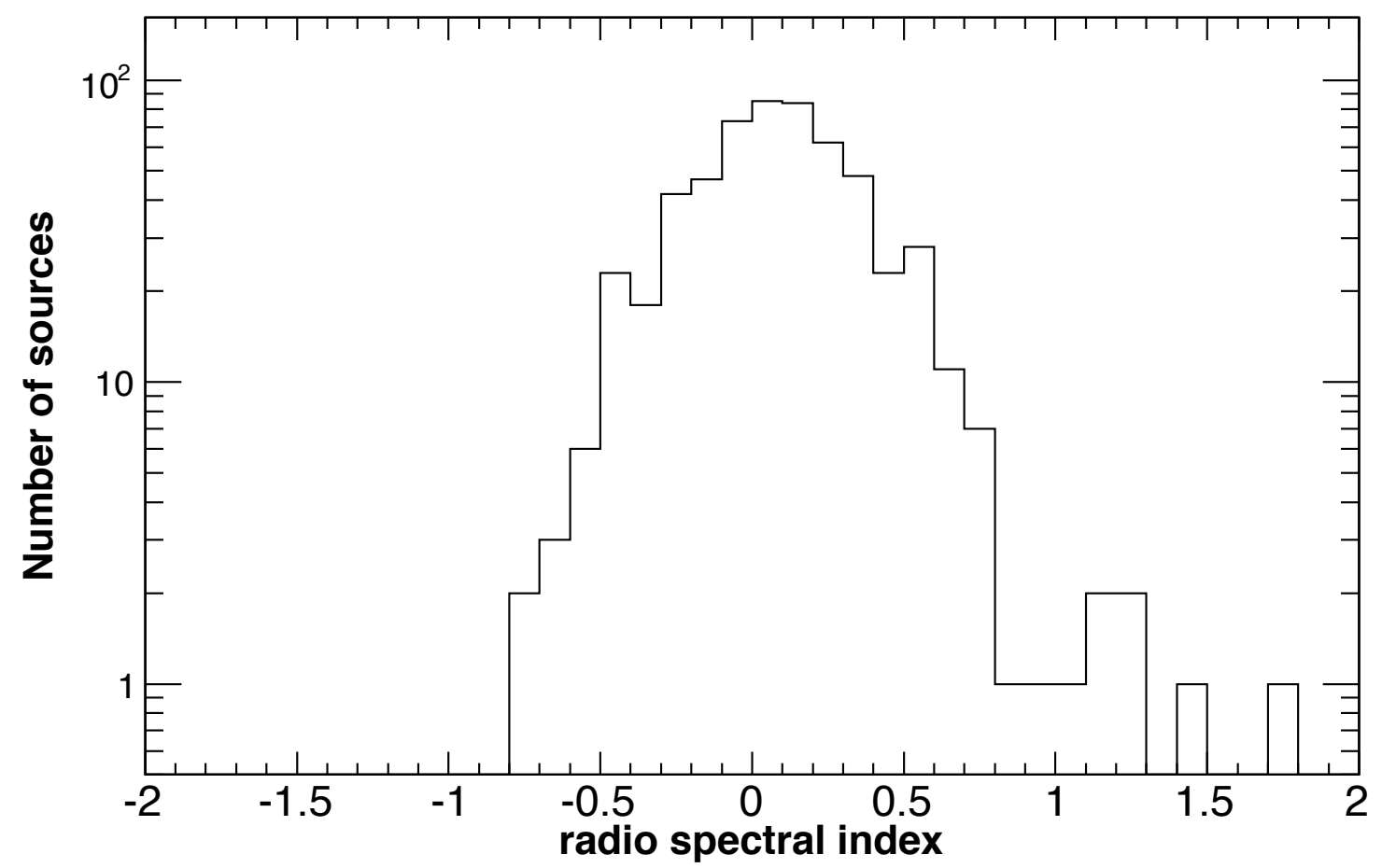

Fig. 28.- Distribution of radio spectral indices between $1.4 \mathrm{GHz}(0.8 \mathrm{GHz}$ for southern sources) and $8.4 \mathrm{GHz}$ for sources in the clean sample. 
Table 1. First LAT AGN Catalog

\begin{tabular}{|c|c|c|c|c|c|c|c|c|c|c|c|c|c|c|c|c|c|c|}
\hline 1FGL name & Associated AGN & $\mathrm{RA}^{\mathrm{a}}$ & $\mathrm{DEC}^{\mathrm{a}}$ & Ang. sep. ${ }^{b}$ & $\theta_{95}{ }^{\mathrm{c}}$ & Assoc. prob. & Opt. class & SED class & $z$ & $F_{35}{ }^{\mathrm{d}}$ & $\Delta F_{35}{ }^{\mathrm{d}}$ & $\Gamma^{\mathrm{d}}$ & $\Delta \Gamma^{\mathrm{d}}$ & $\sigma^{\mathrm{d}}$ & Var.? ${ }^{\mathrm{d}}$ & ${ }^{\mathrm{d}}$ Curv.? ${ }^{\mathrm{d}}$ & ${ }^{d}$ Note $e^{e}$ & Clean? ${ }^{\mathrm{f}}$ \\
\hline 1FGL J0000.9-0745 & CRATES J0001-0746 & 0.32512 & -7.77417 & 0.089 & 0.153 & 0.96 & BLL & LSP & & 1.0 & 0.0 & 2.41 & 0.20 & 5.6 & $\mathrm{~N}$ & $\mathrm{~N}$ & $\mathrm{~s}$ & $\mathrm{Y}$ \\
\hline 1FGL J0004.7-4737 & PKS $0002-478$ & 1.14867 & -47.60517 & 0.032 & 0.153 & 0.99 & FSRQ & LSP & 0.880 & 0.8 & 0.3 & 2.56 & 0.17 & 6.6 & $\mathrm{~N}$ & $\mathrm{~N}$ & $\mathrm{~S}$ & $\mathrm{Y}$ \\
\hline 1FGL J0005.7+3815 & $\mathrm{B} 20003+38 \mathrm{~A}$ & 1.48825 & 38.33755 & 0.088 & 0.200 & 0.99 & FSRQ & LSP & 0.229 & 0.6 & 0.3 & 2.86 & 0.13 & 8.4 & $\mathrm{~N}$ & $\mathrm{~N}$ & $\mathrm{~s}$ & $\mathrm{Y}$ \\
\hline 1FGL J0008.3+1452 & RX J0008.0+1450 & 2.02345 & 14.83982 & 0.072 & 0.143 & 0.70 & AGN & & 0.045 & 0.8 & 0.2 & 2.00 & 0.21 & 4.7 & $\mathrm{~N}$ & $\mathrm{~N}$ & $\mathrm{~s}$ & $\mathrm{~N}$ \\
\hline 1FGL J0008.9+0635 & CRATES J0009+0628 & 2.26638 & 6.47256 & 0.119 & 0.117 & 0.93 & BLL & LSP & & 0.8 & 0.0 & 2.28 & 0.22 & 5.0 & $\mathrm{~N}$ & $\mathrm{~N}$ & $\mathrm{~s}$ & $\mathrm{Y}$ \\
\hline 1FGL J0011.1+0050 & CGRaBS J0011+0057 & 2.87667 & 0.96439 & 0.153 & 0.420 & 0.96 & FSRQ & LSP & 1.492 & 0.6 & 0.2 & 2.51 & 0.15 & 5.8 & $\mathrm{~N}$ & $\mathrm{~N}$ & $\mathrm{~s}$ & $\mathrm{Y}$ \\
\hline 1FGL J0013.1-3952 & PKS $0010-401$ & 3.24962 & -39.90717 & 0.044 & 0.104 & 1.00 & BLL & & & 0.5 & 0.3 & 2.09 & 0.22 & 5.0 & $\mathrm{~N}$ & $\mathrm{~N}$ & $\mathrm{~s}$ & $\mathrm{Y}$ \\
\hline 1FGL J0013.7-5022 & BZB J0014-5022 & 3.54675 & -50.37575 & 0.075 & 0.135 & 1.00 & BLL & HSP & & 0.6 & 0.2 & 2.23 & 0.22 & 4.4 & $\mathrm{~N}$ & $\mathrm{~N}$ & $\mathrm{~s}$ & $\mathrm{Y}$ \\
\hline 1FGL J0017.4-0510 & CGRaBS J0017-0512 & 4.39925 & -5.21158 & 0.040 & 0.079 & 1.00 & FSRQ & LSP & 0.227 & 1.5 & 0.3 & 2.60 & 0.07 & 20.2 & $\mathrm{Y}$ & $\mathrm{Y}$ & $\mathrm{s}$ & $\mathrm{Y}$ \\
\hline 1FGL J0017.7-0019 & PKS 0013-00 & 4.04621 & -0.25347 & 0.390 & 0.315 & 0.57 & FSRQ & LSP & 1.574 & 0.5 & 0.2 & 2.88 & 0.18 & 5.1 & $\mathrm{~N}$ & $\mathrm{~N}$ & $\mathrm{~s}$ & $\mathrm{~N}$ \\
\hline 1FGL J0018.6+2945 & BZB J0018+2947 & 4.61562 & 29.79178 & 0.044 & 0.062 & 1.00 & BLL & HSP & & 0.8 & 0.0 & 1.48 & 0.35 & 6.0 & $\mathrm{~N}$ & $\mathrm{~N}$ & $\mathrm{~s}$ & $\mathrm{Y}$ \\
\hline 1FGL J0019.3+2017 & PKS $0017+200$ & 4.90771 & 20.36267 & 0.100 & 0.177 & 0.99 & BLL & LSP & & 0.7 & 0.2 & 2.38 & 0.15 & 5.9 & $\mathrm{~N}$ & $\mathrm{~N}$ & $\mathrm{~s}$ & $\mathrm{Y}$ \\
\hline 1FGL J0021.7-2556 & CRATES J0021-2550 & 5.38563 & -25.84703 & 0.110 & 0.116 & 0.86 & BLL & ISP & $\ldots$ & 0.9 & 0.2 & 1.96 & 0.17 & 7.3 & $\mathrm{~N}$ & $\mathrm{~N}$ & $\mathrm{~s}$ & $\mathrm{Y}$ \\
\hline 1FGL J0022.5+0607 & PKS $0019+058$ & 5.63517 & 6.13450 & 0.009 & 0.090 & 1.00 & BLL & LSP & $\ldots$ & 1.5 & 0.3 & 2.15 & 0.11 & 10.1 & $\mathrm{~N}$ & $\mathrm{~N}$ & $\mathrm{~S}$ & $\mathrm{Y}$ \\
\hline 1FGL J0023.0+4453 & B3 $0020+446$ & 5.89767 & 44.94328 & 0.116 & 0.120 & 0.96 & FSRQ & & 1.062 & 1.0 & 0.3 & 2.46 & 0.16 & 7.1 & $\mathrm{~N}$ & $\mathrm{~N}$ & $\mathrm{~s}$ & $\mathrm{Y}$ \\
\hline 1FGL J0029.9-4221 & PKS $0027-426$ & 7.57283 & -42.41292 & 0.079 & 0.134 & 0.99 & FSRQ & LSP & 0.495 & 0.7 & 0.2 & 2.39 & 0.17 & 6.6 & $\mathrm{~N}$ & $\mathrm{~N}$ & $\mathrm{~s}$ & $\mathrm{Y}$ \\
\hline 1FGL J0033.5-1921 & RBS 76 & 8.39292 & -19.35944 & 0.006 & 0.053 & 1.00 & BLL & HSP & 0.610 & 2.8 & 0.4 & 1.89 & 0.08 & 17.5 & $\mathrm{~N}$ & $\mathrm{~N}$ & $\mathrm{~s}$ & $\mathrm{Y}$ \\
\hline 1FGL J0035.1+1516 & RX J0035.2+1515 & 8.81125 & 15.25111 & 0.026 & 0.072 & 1.00 & BLL & HSP & & 1.5 & 0.3 & 1.64 & 0.14 & 10.9 & $\mathrm{~N}$ & $\mathrm{~N}$ & $\mathrm{~S}$ & $\mathrm{Y}$ \\
\hline 1FGL J0038.4-2504 & PKS $0035-252$ & 9.56137 & -24.98394 & 0.100 & 0.128 & 0.98 & FSRQ & LSP & 1.196 & 1.0 & 0.3 & 2.45 & 0.13 & 9.3 & $\mathrm{Y}$ & $\mathrm{N}$ & $\mathrm{s}$ & Y \\
\hline 1FGL J0041.9+2318 & PKS $0039+230$ & 10.51896 & 23.33367 & 0.043 & 0.201 & 0.98 & FSRQ & & 1.426 & 0.8 & 0.3 & 2.52 & 0.17 & 5.0 & $\mathrm{~N}$ & $\mathrm{~N}$ & $\mathrm{~s}$ & $\mathrm{Y}$ \\
\hline $1 \mathrm{FGL} \mathrm{J0045.3+2127}$ & BZB J0045+2127 & 11.33042 & 21.46113 & 0.009 & 0.029 & 1.00 & BLL & HSP & & 2.1 & 0.3 & 1.84 & 0.12 & 14.6 & $\mathrm{~N}$ & $\mathrm{~N}$ & $\mathrm{~s}$ & $\mathrm{Y}$ \\
\hline 1FGL J0047.3-2512 & NGC 253 & 11.88806 & -25.28812 & 0.097 & 0.176 & 1.00 & AGN & & 0.001 & 0.7 & 0.2 & 2.15 & 0.17 & 6.2 & $\mathrm{~N}$ & $\mathrm{~N}$ & $\mathrm{~S}$ & $\mathrm{Y}$ \\
\hline 1FGL J0048.0-8412 & PKS 0044-84 & 11.11192 & -84.37781 & 0.191 & 0.229 & 0.96 & FSRQ & $\ldots$ & 1.032 & 1.3 & 0.0 & 2.69 & 0.17 & 5.6 & $\mathrm{~N}$ & $\mathrm{~N}$ & $\mathrm{~s}$ & $\mathrm{Y}$ \\
\hline 1FGL J0048.0-8412 & PKS 0044-84 & 11.11192 & -84.37781 & 0.191 & 0.229 & 0.96 & FSRQ & & 1.032 & 1.3 & 0.0 & 2.69 & 0.17 & 5.6 & $\mathrm{~N}$ & $\mathrm{~N}$ & $\mathrm{~s}$ & Y \\
\hline 1FGL J0049.8-5738 & PKS $0047-579$ & 12.49775 & -57.64067 & 0.019 & 0.070 & 1.00 & FSRQ & LSP & 1.797 & 0.6 & 0.2 & 2.42 & 0.16 & 8.3 & $\mathrm{~N}$ & $\mathrm{~N}$ & $\mathrm{~s}$ & $\mathrm{Y}$ \\
\hline 1FGL J0050.0-0446 & PKS $0047-051$ & 12.58971 & -4.87242 & 0.119 & 0.184 & 0.98 & FSRQ & $\ldots$ & 0.920 & 0.7 & 0.3 & 2.34 & 0.18 & 5.6 & $\mathrm{~N}$ & $\mathrm{~N}$ & $\mathrm{~s}$ & $\mathrm{Y}$ \\
\hline 1FGL J0050.2+0235 & PKS $0047+023$ & 12.43017 & 2.61772 & 0.125 & 0.116 & 0.98 & BLL & & & 0.5 & 0.3 & 2.27 & 0.19 & 5.6 & $\mathrm{~N}$ & $\mathrm{~N}$ & $\mathrm{~S}$ & $\mathrm{Y}$ \\
\hline 1FGL J0050.6-0928 & PKS 0048-09 & 12.67217 & -9.48478 & 0.008 & 0.064 & 1.00 & BLL & ISP & & 4.5 & 0.5 & 2.20 & 0.05 & 27.4 & $\mathrm{Y}$ & $\mathrm{N}$ & $\mathrm{s}$ & $\mathrm{Y}$ \\
\hline 1FGL J0051.1-0649 & PKS 0048-071 & 12.78421 & -6.83394 & 0.016 & 0.105 & 1.00 & FSRQ & LSP & 1.975 & 1.9 & 0.3 & 2.36 & 0.09 & 12.8 & $\mathrm{Y}$ & $\mathrm{N}$ & $\mathrm{S}$ & $\mathrm{Y}$ \\
\hline 1FGL J0058.0+3314 & CRATES J0058+3311 & 14.63363 & 33.18811 & 0.104 & 0.110 & 0.95 & BLL & & 1.371 & 1.7 & 0.3 & 2.33 & 0.11 & 10.5 & Y & $\mathrm{N}$ & $\mathrm{s}$ & $\mathrm{Y}$ \\
\hline 1FGL J0058.4-3235 & PKS $0055-328$ & 14.50925 & -32.57286 & 0.078 & 0.132 & 0.99 & BLL & . & & 0.8 & 0.2 & 2.31 & 0.15 & $\begin{array}{r}7.0 \\
\end{array}$ & $\mathrm{~N}$ & $\mathrm{~N}$ & $\mathrm{~s}$ & $\mathrm{Y}$ \\
\hline $1 \mathrm{FGL} \mathrm{J0100.2+0747}$ & CRATES J0100+0745 & 15.08662 & 7.76428 & 0.048 & 0.047 & 0.97 & Unknown & .. & $\ldots$ & 2.6 & 0.4 & 1.86 & 0.09 & 15.9 & $\mathrm{Y}$ & $\mathrm{N}$ & $\mathrm{s}$ & $\mathrm{Y}$ \\
\hline 1FGL J0102.2+4223 & CRATES J0102+4214 & 15.61313 & 42.23861 & 0.154 & 0.192 & 0.93 & FSRQ & $\ldots$ & 0.874 & 0.6 & 0.3 & 2.74 & 0.15 & 7.8 & $\mathrm{~N}$ & $\mathrm{~N}$ & $\mathrm{~s}$ & $\mathrm{Y}$ \\
\hline 1FGL J0104.4-2406 & PKS $0102-245$ & 16.24250 & -24.27456 & 0.212 & 0.235 & 0.94 & FSRQ & & 1.747 & 1.0 & 0.0 & 2.28 & 0.20 & 4.5 & $\mathrm{~N}$ & $\mathrm{~N}$ & $\mathrm{~s}$ & $\mathrm{Y}$ \\
\hline
\end{tabular}

Note. - The first page of the table is shown here. The full table is available in machine-readable form in the electronic version.

a J2000 coordinate of the associated AGN

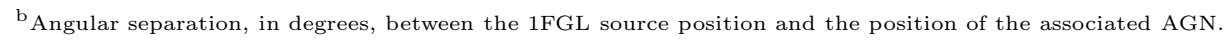

${ }^{\mathrm{c}} \theta_{95} \equiv \sqrt{\theta_{1} \theta_{2}}$, where $\theta_{1}$ and $\theta_{2}$ are the semimajor and semiminor axes (in degrees) of the $\gamma$-ray $95 \%$ confidence region.

${ }^{\mathrm{d}} \gamma$-ray properties from the $1 \mathrm{FGL}$ catalog. $\boldsymbol{F}_{\mathbf{3 5}}$ : The photon flux in units of $10^{-9}$ photons cm ${ }^{-2} \mathrm{~s}^{-1}$ for 1-100 GeV. $\boldsymbol{\Delta} \boldsymbol{F}_{35}: 1 \sigma$ uncertainty on $F_{35}$ in the same units. An entry of "0.0" indicates that the value of $F_{35}$ is an upper limit. $\Gamma$ : Photon number spectral index. $\Delta \Gamma: 1 \sigma$ uncertainty on $\Gamma$. $\sigma$ : Detection significance. Var.?: "Y" indicates a probability < $1 \%$ that the source is steady. Curv.?: "Y" indicates a probability $<1 \%$ that a simple power law is a good fit to the spectrum.

${ }^{\mathrm{e}}$ The codes in this column provide information on multiple associations for a given 1FGL source. S: the 1FGL source is associated with exactly one AGN. MM: the 1FGL source is associated with at least two AGNs with high confidence $(P>0.80)$. Mm: the 1 FGL source is associated with at least one AGN with high confidence $(P>0.80)$ and at least one AGN with lower confidence

$\mathrm{f}$ " $\mathrm{Y}$ " indicates that the source is in the clean sample, defined in Section 3.2 .1 
Table 2. AGN associations for low-latitude $\left(|b|<10^{\circ}\right)$ 1FGL sources

\begin{tabular}{|c|c|c|c|c|c|c|c|c|c|c|c|c|c|c|c|c|c|}
\hline 1FGL name & Associated AGN & $\mathrm{RA}^{\mathrm{a}}$ & $\mathrm{DEC}^{\mathrm{a}}$ & Ang. sep. ${ }^{b}$ & $\theta_{95}{ }^{\mathrm{c}}$ & Assoc. prob. & Opt. class & SED class & $z$ & $F_{35}{ }^{\mathrm{d}}$ & $\Delta F_{35}{ }^{\mathrm{d}}$ & $\Gamma^{\mathrm{d}}$ & $\Delta \Gamma^{\mathrm{d}}$ & $\sigma^{\mathrm{d}}$ & Var. $?^{d}$ & Curv. $?^{\mathrm{d}}$ & Note $^{e}$ \\
\hline 1FGL J0035.9+5951 & 1ES $0033+595$ & 8.96935 & 59.83461 & 0.020 & 0.028 & 1.00 & BLL & $\ldots$ & $\ldots$ & 3.2 & 0.5 & 1.95 & 0.20 & 13.0 & $\mathrm{~N}$ & $\mathrm{~N}$ & $\mathrm{~S}$ \\
\hline 1FGL J0046.8+5658 & VCS1 J0047+5657 & $\begin{array}{r}0.90530 \\
11.75179\end{array}$ & 56.96178 & 0.023 & 0.115 & 0.99 & Unknown & $\ldots$ & $\ldots$ & 2.3 & 0.4 & $\begin{array}{l}2.90 \\
2.27\end{array}$ & 0.17 & $\begin{array}{l}10.0 \\
7.5\end{array}$ & $\mathrm{~N}$ & $\mathrm{~N}$ & $\mathrm{~S}$ \\
\hline 1FGL J0102.8+5827 & TXS $0059+581$ & 15.69068 & 58.40309 & 0.064 & 0.079 & 0.99 & FSRQ & $\ldots$ & 0.644 & 4.2 & 0.5 & 2.38 & 0.13 & 16.3 & $\mathrm{Y}$ & $\mathrm{N}$ & $\mathrm{s}$ \\
\hline 1FGL J0110.0+6806 & $4 \mathrm{C}+67.04$ & 17.55364 & 68.09478 & $\begin{array}{l}0.021 \\
0.021\end{array}$ & 0.074 & 1.00 & Unknown & $\ldots$ & & 2.0 & 0.5 & $\begin{array}{l}2.00 \\
2.35\end{array}$ & 0.21 & 8.5 & $\mathrm{~N}$ & $\mathrm{~N}$ & $\mathrm{~s}^{3}$ \\
\hline 1FGL J0254.2+5107 & TXS $0250+508$ & 43.49003 & 51.04902 & 0.089 & 0.114 & 0.99 & Unknown & $\ldots$ & $\ldots$ & 2.3 & 0.5 & 2.42 & 0.22 & 9.3 & $\mathrm{~N}$ & $\mathrm{~N}$ & $\mathrm{~s}$ \\
\hline 1FGL J0303.1+4711 & $4 \mathrm{C}+47.08$ & 45.89684 & 47.27119 & $\begin{array}{l}0.009 \\
0.109\end{array}$ & 0.149 & $\begin{array}{l}.95 \\
1.00\end{array}$ & BLL & $\ldots$ & $\ldots$ & 1.4 & 0.4 & 2.56 & 0.15 & 6.8 & $\mathrm{~N}$ & $\mathrm{~N}$ & $\mathrm{~s}$ \\
\hline 1FGL J0334.3+6536 & RX J0333.9+6537 & 53.48641 & 65.61561 & 0.047 & 0.094 & 0.99 & Unknown & $\ldots$ & $\ldots$ & 1.3 & 0.5 & 2.19 & 0.22 & 4.1 & $\mathrm{~N}$ & $\mathrm{~N}$ & $\mathrm{~s}$ \\
\hline 1FGL J0419.0+3811 & $3 \mathrm{C} 111$ & 64.58866 & 38.02661 & 0.214 & 0.255 & 0.87 & AGN & $\ldots$ & 0.049 & 1.5 & 0.5 & 2.61 & 0.22 & 4.3 & $\mathrm{~N}$ & $\mathrm{~N}$ & $\mathrm{~s}$ \\
\hline 1FGL J0423.8+4148 & $4 \mathrm{C}+41.11$ & 65.98337 & 41.83409 & 0.025 & 0.031 & 1.00 & Unknown & $\ldots$ & $\ldots$ & 3.5 & 0.5 & 1.87 & 0.07 & 15.2 & $\mathrm{~N}$ & $\mathrm{~N}$ & $\mathrm{~s}$ \\
\hline 1FGL J0521.7+2114 & RX J0521.7+2112 & 80.44152 & 21.21429 & 0.021 & 0.030 & 1.00 & Unknown & $\ldots$ & & 5.5 & 0.6 & 1.94 & 0.18 & 21.1 & $\mathrm{~N}$ & $\mathrm{~N}$ & $\mathrm{~S}$ \\
\hline 1FGL J0533.0+4825 & RX J0533.2+4823 & 83.31611 & 48.38134 & 0.054 & 0.110 & 1.00 & FSRQ & $\ldots$ & 1.162 & 2.3 & 0.4 & 2.43 & 0.35 & 10.3 & $\mathrm{Y}$ & $\mathrm{N}$ & $\mathrm{S}$ \\
\hline 1FGL J0648.7-1740 & TXS $0646-176$ & 102.11874 & -17.73484 & 0.092 & 0.107 & 0.99 & FSRQ & $\ldots$ & 1.232 & 2.5 & 0.5 & 2.47 & 0.15 & 10.8 & $\mathrm{Y}$ & $\mathrm{N}$ & $\mathrm{s}$ \\
\hline 1FGL J0650.6-1635 & PKS $0648-16$ & 102.60242 & $\begin{array}{l}-16.62770 \\
-\end{array}$ & 0.075 & 0.151 & 0.98 & Unknown & $\ldots$ & $\begin{array}{l}1.252 \\
\ldots\end{array}$ & 1.4 & 0.4 & 2.46 & 0.17 & $\begin{array}{r}5.8 \\
5.8\end{array}$ & $\mathrm{~N}$ & $\mathrm{~N}$ & $\mathrm{~s}$ \\
\hline 1FGL J0656.2-0321 & $\mathrm{OH}-090$ & 104.04634 & -3.38522 & 0.034 & 0.077 & 1.00 & Unknown & $\ldots$ & $\ldots$ & 3.9 & 0.6 & 2.59 & 0.11 & 16.1 & $\mathrm{Y}$ & $\mathrm{N}$ & $\mathrm{s}$ \\
\hline 1FGL J0702.2-1954 & TXS $0700-197$ & 105.67875 & $\begin{array}{r}-3.00022 \\
-19.85612\end{array}$ & $\begin{array}{l}0.034 \\
0.120\end{array}$ & 0.159 & 0.96 & Unknown & $\ldots$ & $\ldots$ & $\begin{array}{l}3.9 \\
1.7\end{array}$ & $\begin{array}{l}0.0 \\
0.4\end{array}$ & 1.92 & $\begin{array}{l}0.11 \\
0.16\end{array}$ & $\begin{array}{r}10.1 \\
4.2\end{array}$ & $\begin{array}{l}1 \\
\mathrm{~N}\end{array}$ & $\mathrm{~N}$ & S \\
\hline 1FGL J0721.4+0401 & RX J0721.3+0406 & 110.34963 & 4.11228 & 0.083 & 0.125 & 0.98 & Unknown & $\ldots$ & 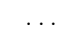 & 0.8 & 0.3 & 2.68 & 0.17 & 6.8 & $\mathrm{~N}$ & $\mathrm{~N}$ & $\mathrm{~s}$ \\
\hline 1FGL J0725.9-0053 & PKS 0723-008 & 111.46100 & -0.91571 & 0.043 & 0.077 & 1.00 & BLL & $\ldots$ & 0.128 & 0.6 & 0.3 & 2.30 & 0.08 & 6.1 & $\mathrm{~N}$ & $\mathrm{~N}$ & $\mathrm{~S}$ \\
\hline 1FGL J0730.3-1141 & PKS $0727-11$ & 112.57963 & -11.68683 & 0.013 & 0.022 & 1.00 & FSRQ & $\ldots$ & 1.591 & 20.7 & 1.0 & 2.33 & 0.14 & 67.6 & $\mathrm{Y}$ & $\mathrm{N}$ & $\mathrm{S}$ \\
\hline 1FGL J0754.4-1147 & OI -187 & 118.61024 & -11.78804 & 0.009 & 0.068 & 1.00 & Unknown & $\ldots$ & & 1.9 & 0.4 & 2.10 & 0.13 & 10.5 & $\mathrm{~N}$ & $\mathrm{~N}$ & $\mathrm{~s}$ \\
\hline 1FGL J0825.8-2230 & PKS $0823-223$ & 126.50655 & -22.50756 & 0.036 & 0.038 & 1.00 & BLL & $\ldots$ & $\ldots$ & $\begin{array}{l}5.3 \\
5.3\end{array}$ & 0.5 & 2.14 & 0.17 & 26.5 & $\mathrm{~N}$ & $\mathrm{~N}$ & $\mathrm{~s}$ \\
\hline 1FGL J0825.9-3216 & PKS $0823-321$ & 126.46405 & -32.30645 & 0.036 & 0.110 & 1.00 & Unknown & $\ldots$ & $\ldots$ & 1.4 & 0.4 & 2.68 & 0.12 & 8.8 & $\mathrm{Y}$ & $\mathrm{N}$ & $\mathrm{s}$ \\
\hline 1FGL J0827.9-3738 & PKS B0826-373 & 127.01992 & -37.51841 & 0.134 & 0.194 & 0.96 & Unknown & $\ldots$ & $\ldots$ & 1.4 & 0.6 & $\begin{array}{l}2.00 \\
2.68\end{array}$ & 0.17 & $\begin{array}{l}0.0 \\
5.7\end{array}$ & $\mathrm{Y}$ & $\mathrm{N}$ & $\mathrm{s}$ \\
\hline 1FGL J0845.0-5459 & PMN J0845-5458 & 131.26034 & -54.96904 & 0.020 & 0.101 & 0.99 & Unknown & $\ldots$ & $\ldots$ & 1.9 & 0.4 & 2.24 & 0.17 & 7.7 & $\mathrm{~N}$ & $\mathrm{~N}$ & $\mathrm{~s}$ \\
\hline 1FGL J0849.6-3540 & 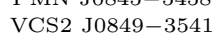 & 132.44010 & $\begin{array}{l}-34.90904 \\
-35.68369\end{array}$ & $\begin{array}{l}0.020 \\
0.023\end{array}$ & $\begin{array}{l}0.101 \\
0.152\end{array}$ & 0.98 & Unknown & $\ldots$ & $\ldots$ & $\begin{array}{l}1.9 \\
1.9\end{array}$ & $\begin{array}{l}0.4 \\
0.4\end{array}$ & $\begin{array}{l}2.24 \\
2.40\end{array}$ & 0.16 & 6.0 & $\mathrm{~N}$ & $\mathrm{~N}$ & S \\
\hline 1FGL J0905.1-5736 & PKS $0903-57$ & 136.22158 & -57.58494 & 0.041 & 0.068 & 1.00 & FSRQ & $\ldots$ & 0.695 & 1.8 & 0.4 & 2.36 & 0.18 & 7.5 & $\mathrm{Y}$ & $\mathrm{N}$ & $\mathrm{s}$ \\
\hline 1FGL J1103.9-5355 & PKS 1101-536 & $\begin{array}{l}150.22100 \\
165.96759\end{array}$ & $\begin{array}{l}-53.00494 \\
-53.95019\end{array}$ & $\begin{array}{l}0.041 \\
0.019\end{array}$ & 0.041 & $\begin{array}{l}1.00 \\
1.00\end{array}$ & Unknown & $\ldots$ & & $\begin{array}{l}1.0 \\
6.1\end{array}$ & $\begin{array}{l}0.4 \\
0.6\end{array}$ & $\begin{array}{l}2.50 \\
2.05\end{array}$ & $\begin{array}{l}0.10 \\
0.19\end{array}$ & 19.5 & $\begin{array}{l}1 \\
\text { Y }\end{array}$ & $\mathrm{N}$ & S \\
\hline 1FGL J1122.9-6415 & PMN J1123-6417 & 170.83090 & -64.29339 & 0.059 & 0.088 & 0.99 & Unknown & $\ldots$ & $\ldots$ & 2.4 & 0.0 & 2.48 & 0.05 & 4.7 & $\mathrm{Y}$ & $\mathrm{N}$ & $\mathrm{s}$ \\
\hline 1FGL J1307.3-6701 & PKS 1304-668 & 197.07240 & -67.11812 & 0.129 & 0.152 & 0.92 & Unknown & $\ldots$ & $\ldots$ & 1.4 & 0.5 & 2.57 & 0.09 & 5.3 & $\mathrm{Y}$ & $\mathrm{N}$ & $\mathrm{s}$ \\
\hline 1FGL J1327.0-5257 & PMN J1326-5256 & 201.70512 & -52.93990 & 0.040 & 0.061 & $\begin{array}{l}.02 \\
1.00\end{array}$ & Unknown & $\ldots$ & $\ldots$ & $\begin{array}{l}1.4 \\
5.2\end{array}$ & 0.6 & 2.33 & 0.11 & $\begin{array}{r}0.0 \\
19.2\end{array}$ & $\mathrm{Y}$ & $\mathrm{N}$ & $\mathrm{s}$ \\
\hline 1FGL J1329.2-5605 & PMN J1329-5608 & 202.25477 & -56.13407 & 0.055 & 0.095 & 1.00 & Unknown & $\ldots$ & $\ldots$ & 4.1 & 0.6 & 2.56 & 0.15 & 15.1 & $\mathrm{Y}$ & $\mathrm{N}$ & $\mathrm{s}$ \\
\hline 1FGL J1330.7-7006 & PKS 1326-697 & 202.54615 & -70.05363 & 0.072 & 0.114 & 0.99 & Unknown & $\ldots$ & $\ldots$ & $\begin{array}{l}4.1 \\
1.5\end{array}$ & 0.4 & 2.46 & 0.09 & $\begin{array}{r}10.1 \\
8.3\end{array}$ & Y & $\mathrm{N}$ & $\mathrm{s}$ \\
\hline 1FGL J1400.9-5559 & PMN J1400-5605 & 210.17407 & -56.08210 & 0.092 & 0.122 & 0.99 & Unknown & $\ldots$ & $\ldots$ & 2.4 & 0.5 & 2.62 & 0.15 & 9.1 & $\mathrm{Y}$ & $\mathrm{N}$ & $\mathrm{s}$ \\
\hline 1FGL J1514.1-4745 & PMN J1514-4748 & 228.66677 & $\begin{array}{l}-50.08210 \\
-4780829\end{array}$ & 0.096 & 0.124 & 0.98 & Unknown & $\ldots$ & $\ldots$ & $\begin{array}{l}2.4 \\
1.2\end{array}$ & 0.4 & $\begin{array}{l}2.02 \\
2.29\end{array}$ & $\begin{array}{l}0.10 \\
0.20\end{array}$ & 5.7 & $\begin{array}{l}1 \\
\mathrm{~N}\end{array}$ & $\mathrm{~N}$ & S \\
\hline 1FGL J1603.8-4903 & PMN J1603-4904 & 240.96119 & -49.06820 & 0.007 & 0.027 & 1.00 & Unknown & $\ldots$ & $\ldots$ & 13.4 & 1.1 & 2.12 & 0.14 & 26.8 & $\mathrm{~N}$ & $\mathrm{Y}$ & $\mathrm{s}$ \\
\hline 1FGL J1604.7-4443 & PMN J1604-4441 & 241.12925 & -44.69221 & 0.046 & 0.066 & 1.00 & Unknown & $\ldots$ & $\ldots$ & 7.7 & 0.8 & 2.46 & 0.04 & 22.2 & $\mathrm{Y}$ & $\mathrm{N}$ & $\mathrm{S}$ \\
\hline
\end{tabular}

Note. - The first page of the table is shown here. The full table is available in machine-readable form in the electronic version.

${ }^{a}$ J2000 coordinate of the associated AGN.

${ }^{\mathrm{b}}$ Angular separation, in degrees, between the $1 \mathrm{FGL}$ source position and the position of the associated AGN.

${ }^{\mathrm{c}} \theta_{95} \equiv \sqrt{\theta_{1} \theta_{2}}$, where $\theta_{1}$ and $\theta_{2}$ are the semimajor and semiminor axes (in degrees) of the $\gamma$-ray $95 \%$ confidence region.

${ }^{\mathrm{d}} \gamma$-ray properties from the $1 \mathrm{FGL}$ catalog. $\boldsymbol{F}_{35}$ : The photon flux in units of $10^{-9}$ photons $\mathrm{cm}^{-2} \mathrm{~s}^{-1}$ for 1-100 GeV. $\boldsymbol{\Delta} \boldsymbol{F}_{35}$ : $1 \sigma$ uncertainty on $F_{35}$ in the same units. An entry of "0.0" indicates that the value of $F_{35}$ is an upper limit. $\Gamma$ : Photon number spectral index. $\Delta \Gamma: 1 \sigma$ uncertainty on $\Gamma$. $\sigma$ : Detection significance. Var.?: "Y" indicates a probability $<1 \%$ that the source is steady. Curv.?: "Y" indicates a probability $<1 \%$ that a simple power law is a good fit to the spectrum.
Cut the

${ }^{\mathrm{e}}$ The codes in this column provide information on multiple associations for a given $1 \mathrm{FGL}$ source. S: the 1FGL source is associated with exactly one AGN. MM: the 1FGL source is associated with at least two AGNs with high confidence $(P>0.80)$. Mm: the 1FGL source is associated with at least one AGN with high confidence $(P>0.80)$ and at least one AGN with lower confidence
$(0.50<P<0.80)$. mm: the 1 FGL source is associated with at least two AGNs with lower confidence $(0.50<P<0.80)$. 
Table 3. AGN "affiliations" for 1FGL sources

\begin{tabular}{|c|c|c|c|c|c|c|c|c|c|c|c|c|c|c|c|}
\hline 1FGL name & Associated AGN & $\mathrm{RA}^{\mathrm{a}}$ & $\mathrm{DEC}^{\mathrm{a}}$ & Ang. sep. ${ }^{b}$ & $\theta_{95}{ }^{\mathrm{c}}$ & Opt. class & SED class & $z$ & $F_{35}{ }^{\mathrm{d}}$ & $\Delta F_{35}{ }^{\mathrm{d}}$ & $\Gamma^{\mathrm{d}}$ & $\Delta \Gamma^{\mathrm{d}}$ & $\sigma^{\mathrm{d}}$ & Var. $?^{\mathrm{d}}$ & Curv.? ${ }^{\mathrm{d}}$ \\
\hline 1FGL J0016.6+1706 & CRATES J0015+1700 & 3.91662 & 17.01128 & 0.247 & 0.193 & FSRQ & 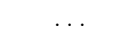 & 1.716 & 0.5 & 0.3 & 2.57 & 0.20 & 4.7 & $\mathrm{~N}$ & $\mathrm{~N}$ \\
\hline 1FGL J0022.2-1850 & 1RXS J002209.2-185333 & 5.53800 & -18.89248 & 0.054 & 0.111 & Unknown & HSP & & 1.1 & 0.3 & 1.56 & 0.14 & 9.4 & $\mathrm{~N}$ & $\mathrm{~N}$ \\
\hline 1FGL J0024.6+0346 & CLASS J0024+0349 & 6.18841 & 3.81766 & 0.045 & 0.099 & FSRQ & $\ldots$ & 0.545 & 0.9 & 0.3 & 2.33 & 0.12 & 8.9 & Y & $\mathrm{N}$ \\
\hline 1FGL J0038.0+1236 & FRBA J0037+1238 & 9.46180 & 12.63863 & 0.060 & 0.098 & Unknown & $\ldots$ & & 0.7 & 0.3 & 2.30 & 0.18 & 7.0 & $\mathrm{~N}$ & $\mathrm{~N}$ \\
\hline 1FGL J0043.6+3424 & CLASS J0043+3426 & 10.95352 & 34.44059 & 0.042 & 0.063 & Unknown & $\ldots$ & & 1.7 & 0.3 & 2.09 & 0.10 & 12.4 & $\mathrm{~N}$ & $\mathrm{~N}$ \\
\hline 1FGL J0048.0+2232 & CLASS J0048+2235 & 12.01092 & 22.59006 & 0.050 & 0.089 & FSRQ & $\ldots$ & 1.156 & 1.5 & 0.3 & 2.39 & 0.09 & 13.5 & $\mathrm{Y}$ & $\mathrm{N}$ \\
\hline 1FGL J0051.4-6242 & RBS 119 & 12.81952 & -62.70120 & 0.025 & 0.067 & Unknown & $\ldots$ & $\ldots$ & 1.8 & 0.3 & 1.68 & 0.12 & 12.0 & $\mathrm{~N}$ & $\mathrm{~N}$ \\
\hline 1FGL J0054.9-2455 & FRBA J0054-2455 & 13.69480 & $\begin{array}{l}-02.0120 \\
-24.92519\end{array}$ & 0.035 & 0.078 & Unknown & HSP & $\ldots$ & $\begin{array}{l}1.0 \\
0.7\end{array}$ & $\begin{array}{l}0.3 \\
0.2\end{array}$ & $\begin{array}{l}1.08 \\
1.95\end{array}$ & $\begin{array}{l}0.12 \\
0.22\end{array}$ & 5.2 & $\mathrm{~N}$ & $\mathrm{~N}$ \\
\hline 1FGL J0110.0-4023 & RBS 158 & 17.48483 & -40.34794 & 0.051 & 0.079 & Unknown & HSP & $\ldots$ & 0.8 & 0.0 & 1.34 & 0.32 & 4.2 & $\mathrm{~N}$ & $\mathrm{~N}$ \\
\hline 1FGL J0115.7+0357 & CLASS J0115+0356 & 18.91880 & 3.94536 & 0.021 & 0.076 & BLL & & $\cdots$ & 1.4 & 0.3 & 2.07 & 0.15 & 8.7 & $\mathrm{~N}$ & $\mathrm{~N}$ \\
\hline 1FGL J0124.6-0616 & AT20G J0124-0625 & 21.21033 & -6.41722 & 0.146 & 0.163 & BLL & $\ldots$ & $\ldots$ & 0.6 & 0.3 & 2.20 & 0.17 & 5.5 & $\mathrm{~N}$ & $\mathrm{~N}$ \\
\hline 1FGL J0134.4+2632 & RX J0134.4+2638 & 23.61779 & 26.64588 & 0.103 & 0.155 & Unknown & HSP & $\ldots$ & 0.9 & 0.3 & 2.26 & 0.16 & 6.6 & $\mathrm{~N}$ & $\mathrm{~N}$ \\
\hline 1FGL J0157.0-5259 & RBS 259 & 29.23839 & -53.03285 & 0.049 & 0.100 & Unknown & $\ldots$ & $\ldots$ & 0.8 & 0.2 & 1.85 & 0.20 & 7.6 & $\mathrm{~N}$ & $\mathrm{~N}$ \\
\hline 1FGL J0217.9-6630 & CRATES J0216-6636 & 34.21187 & -66.61169 & 0.153 & 0.108 & BLL & $\ldots$ & $\ldots$ & 0.9 & 0.3 & 1.94 & 0.17 & 7.0 & $\mathrm{~N}$ & $\mathrm{~N}$ \\
\hline 1FGL J0226.3+0937 & FRBA J0226+0937 & 36.55704 & 9.62398 & 0.034 & 0.133 & Unknown & $\ldots$ & $\ldots$ & 0.9 & 0.3 & $\begin{array}{l}1.99 \\
1.99\end{array}$ & 0.17 & 6.4 & $\mathrm{~N}$ & $\mathrm{~N}$ \\
\hline 1FGL J0256.9+2920 & FRBA J0256+2924 & 44.22623 & 29.41500 & 0.066 & 0.105 & AGN & $\ldots$ & 0.190 & 0.6 & 0.3 & 1.69 & 0.30 & 4.2 & $\mathrm{~N}$ & $\mathrm{~N}$ \\
\hline 1FGL J0315.6-5109 & AT20G J0314-5104 & 48.60733 & -51.07547 & 0.218 & 0.314 & BLL & $\ldots$ & & 0.7 & 0.3 & 2.60 & 0.20 & 6.0 & $\mathrm{~N}$ & $\mathrm{~N}$ \\
\hline 1FGL J0318.1+0254 & CLASS J0317+0248 & 49.44965 & 2.81183 & 0.135 & 0.165 & FSRQ & $\ldots$ & 0.748 & 0.8 & 0.3 & 2.20 & 0.18 & 4.1 & $\mathrm{~N}$ & $\mathrm{~N}$ \\
\hline 1FGL J0333.7+2919 & FRBA J0333+2916 & 53.45420 & $\begin{array}{r}2.01100 \\
29.27542\end{array}$ & 0.057 & 0.044 & Unknown & ISP & & $\begin{array}{l}.0 \\
1.0\end{array}$ & 0.3 & 1.57 & 0.21 & 6.5 & $\mathrm{~N}$ & $\mathrm{~N}$ \\
\hline 1FGL J0342.2+3859 & CLASS J0342+3859 & 55.56779 & 38.98507 & 0.012 & 0.102 & FSRQ & $\ldots$ & 0.945 & 1.1 & 0.0 & 2.17 & 0.29 & 4.7 & $\mathrm{~N}$ & $\mathrm{~N}$ \\
\hline 1FGL J0401.3-3152 & PKS $0400-319$ & 60.58863 & -31.79053 & 0.232 & 0.155 & FSRQ & $\ldots$ & 1.288 & 0.6 & 0.3 & 2.37 & 0.17 & 6.0 & $\mathrm{~N}$ & $\mathrm{~N}$ \\
\hline 1FGL J0445.2-6008 & AT20G J0445-6015 & 71.25667 & -60.25006 & 0.105 & 0.169 & AGN & $\ldots$ & 0.097 & 0.8 & 0.3 & 1.98 & 0.29 & 4.6 & $\mathrm{~N}$ & $\mathrm{~N}$ \\
\hline 1FGL J0506.9-5435 & RBS 621 & 76.74127 & -54.58422 & 0.014 & 0.058 & Unknown & $\ldots$ & & 1.0 & 0.0 & 1.42 & 0.31 & 6.7 & $\mathrm{~N}$ & $\mathrm{~N}$ \\
\hline 1FGL J0515.2+7355 & CLASS J0516+7351 & 79.12993 & 73.85240 & 0.115 & 0.167 & BLL & $\ldots$ & 0.249 & 0.7 & 0.3 & 2.04 & 0.25 & 5.0 & $\mathrm{~N}$ & $\mathrm{~N}$ \\
\hline 1FGL J0515.9+1528 & CLASS J0515+1527 & 78.94732 & 15.45461 & 0.032 & 0.069 & BLL & $\ldots$ & $\ldots$ & 1.2 & 0.4 & 2.01 & 0.18 & 8.4 & $\mathrm{~N}$ & $\mathrm{~N}$ \\
\hline 1FGL J0517.6+0857 & CLASS J0517+0858 & 79.41693 & $\begin{array}{r}10.40401 \\
8.97661\end{array}$ & 0.025 & 0.109 & $\begin{array}{l}\text { DSRQ } \\
\text { FSR }\end{array}$ & $\ldots$ & 0.328 & 1.1 & 0.4 & 2.58 & 0.12 & $\begin{array}{l}8.4 \\
8.5\end{array}$ & $\mathrm{~N}$ & $\mathrm{~N}$ \\
\hline 1FGL J0537.7-5717 & PKS 0541-834 & 84.45325 & -57.30806 & 0.014 & 0.073 & FSRQ & $\ldots$ & $\ldots$ & 1.0 & 0.0 & 1.81 & 0.42 & 6.1 & $\mathrm{~N}$ & $\mathrm{~N}$ \\
\hline 1FGL J0538.4-3910 & 1RXS J053810.0-390839 & 84.54304 & $\begin{array}{l}-01.00000 \\
-39.14562\end{array}$ & $\begin{array}{l}0.014 \\
0.059\end{array}$ & 0.077 & Unknown & HSP & $\ldots$ & 1.0 & 0.0 & $\begin{array}{l}1.01 \\
2.16\end{array}$ & $\begin{array}{l}0.42 \\
0.12\end{array}$ & $\begin{array}{l}0.1 \\
9.0\end{array}$ & $\mathrm{~N}$ & $\mathrm{~N}$ \\
\hline 1FGL J0541.9-0204 & CRATES J0541-0154 & 85.47800 & -2.07800 & 0.011 & 0.075 & Unknown & $\ldots$ & $\ldots$ & 1.5 & 0.6 & 2.30 & 0.12 & 6.0 & $\mathrm{~N}$ & $\mathrm{~N}$ \\
\hline 1FGL J0600.5-2006 & CRATES J0601-2004 & 90.47012 & -20.07922 & 0.303 & 0.260 & FSRQ & $\ldots$ & 1.216 & 0.7 & 0.3 & 2.41 & 0.15 & 6.2 & $\mathrm{~N}$ & $\mathrm{~N}$ \\
\hline 1FGL J0603.0-4012 & 1WGA J0602.8-4018 & 90.71162 & -40.31417 & 0.109 & 0.143 & Unknown & $\ldots$ & $\ldots$ & 1.2 & 0.3 & 2.25 & 0.16 & 5.6 & $\mathrm{~N}$ & $\mathrm{~N}$ \\
\hline 1FGL J0604.2-4817 & 1ES $0602-482$ & 91.03918 & -48.29059 & 0.011 & 0.062 & Unknown & $\ldots$ & $\ldots$ & 1.1 & 0.3 & 2.12 & 0.16 & 7.2 & $\mathrm{~N}$ & $\mathrm{~N}$ \\
\hline 1FGL J0605.1+0005 & CLASS J0604+0000 & 91.24341 & 0.01204 & 0.100 & 0.129 & Unknown & $\ldots$ & $\ldots$ & 1.1 & 0.3 & 1.94 & 0.23 & 5.4 & $\mathrm{~N}$ & $\mathrm{~N}$ \\
\hline 1FGL J0608.1-0630 & CRATES J0609-0615 & 92.41654 & -6.25161 & 0.457 & 0.306 & Unknown & $\ldots$ & $\ldots$ & 1.8 & 0.5 & 2.54 & 0.10 & 8.1 & $\mathrm{~N}$ & $\mathrm{Y}$ \\
\hline 1FGL J0609.3-0244 & NVSS J060915-024754 & 92.31252 & $\begin{array}{l}-0.20101 \\
-2.79840\end{array}$ & 0.065 & 0.096 & Unknown & HSP & $\ldots$ & $\begin{array}{l}1.0 \\
1.2\end{array}$ & $\begin{array}{l}0.5 \\
0.3\end{array}$ & $\begin{array}{l}2.04 \\
2.02\end{array}$ & 0.20 & $\begin{array}{l}0.1 \\
5.9\end{array}$ & $\mathrm{~N}$ & $\mathrm{~N}$ \\
\hline 1FGL J0622.2+3751 & CLASS J0621+3750 & 95.49016 & 37.84916 & 0.058 & 0.131 & Unknown & & $\ldots$ & 2.0 & 0.4 & 2.36 & 0.09 & 10.2 & $\mathrm{~N}$ & $\mathrm{Y}$ \\
\hline 1FGL J0706.5+3744 & CLASS J0706+3744 & $\begin{array}{r}9.49010 \\
106.63207\end{array}$ & $\begin{array}{l}31.04910 \\
37.74344\end{array}$ & 0.007 & $\begin{array}{l}0.101 \\
0.063\end{array}$ & BLL & HSP & $\ldots$ & 1.1 & $\begin{array}{l}0.4 \\
0.3\end{array}$ & 2.01 & 0.09 & $\begin{array}{r}10.2 \\
8.2\end{array}$ & $\mathrm{~N}$ & $\mathrm{~N}$ \\
\hline 1FGL J0707.3+7742 & FRBA J0706+7741 & 106.71367 & 77.69349 & 0.027 & 0.088 & Unknown & $\ldots$ & $\ldots$ & 1.4 & 0.3 & 2.30 & 0.13 & 10.5 & $\mathrm{~N}$ & $\mathrm{~N}$ \\
\hline 1FGL J0723.6+2908 & CLASS J0723+2859 & 110.97850 & 28.99163 & 0.165 & 0.215 & Unknown & ... & $\ldots$ & 0.9 & 0.3 & 2.10 & 0.17 & 5.8 & $\mathrm{Y}$ & $\mathrm{N}$ \\
\hline
\end{tabular}

Note. - The first page of the table is shown here. The full table is available in machine-readable form in the electronic version.

a J2000 coordinate of the associated AGN.

${ }^{\mathrm{b}}$ Angular separation, in degrees, between the 1FGL source position and the position of the associated AGN.

${ }^{\mathrm{c}} \theta_{95} \equiv \sqrt{\theta_{1} \theta_{2}}$, where $\theta_{1}$ and $\theta_{2}$ are the semimajor and semiminor axes (in degrees) of the $\gamma$-ray $95 \%$ confidence region.

$\mathrm{d} \gamma$-ray properties from the $1 \mathrm{FGL}$ catalog. $\boldsymbol{F}_{\mathbf{3 5}}$ : The photon flux in units of $10^{-9}$ photons $\mathrm{cm}^{-2} \mathrm{~s}^{-1}$ for $1-100 \mathrm{GeV}$. $\boldsymbol{\Delta} \boldsymbol{F}_{\mathbf{3 5}}: 1 \sigma$ uncertainty on $F_{35}$ in the same units. An entry of " $0.0^{\text {" }}$ indicates that the value of $F_{35}$ is an upper limit. $\Gamma$ : Photon number spectral index. $\Delta \Gamma$ : $1 \sigma$ uncertainty on $\Gamma$. $\sigma$ : Detection significance. Var.?: "Y" indicates a probability $<1 \%$ that the source is steady. Curv.?: "Y" indicates a probability $<1 \%$ that a simple power law is a good fit to the spectrum. 
Table 4. Census of 1LAC sources

\begin{tabular}{lccc}
\hline \hline & \multicolumn{3}{c}{ Number of AGNs in: } \\
AGN type & Entire 1 LAC sample & High-confidence sample ${ }^{\mathrm{a}}$ & Clean sample $^{\mathrm{a}}$ \\
\hline All & $\mathbf{7 0 9}$ & $\mathbf{6 6 3}$ & $\mathbf{5 9 9}$ \\
& & & \\
FSRQ & $\mathbf{2 9 6}$ & $\mathbf{2 8 1}$ & $\mathbf{2 4 8}$ \\
$\ldots$ LSP & 189 & 185 & 171 \\
$\ldots$. ISP & 3 & 2 & 1 \\
$\ldots$ HSP & 2 & 2 & 1 \\
& & & \\
BL Lac & $\mathbf{3 0 0}$ & $\mathbf{2 9 1}$ & $\mathbf{2 7 5}$ \\
$\ldots$ LSP & 69 & 67 & 62 \\
$\ldots$ ISP & 46 & 44 & 44 \\
$\ldots$ HSP & 118 & 117 & $\mathbf{2 6}$ \\
Other AGN & $\mathbf{4 1}$ & $\mathbf{3 0}$ & $\mathbf{5 0}$ \\
Unknown & $\mathbf{7 2}$ & $\mathbf{6 1}$ & \\
\hline
\end{tabular}

${ }^{\text {a }}$ See Section 3.2.1 for the definitions of these samples. 
Table 5. Positional coincidences of 1LAC sources with TeV sources

\begin{tabular}{|c|c|c|c|}
\hline LAT Source & TeV Association & $\mathrm{RA}^{\mathrm{a}}$ & $\mathrm{DEC}^{\mathrm{a}}$ \\
\hline 1FGL J0222.6+4302 & $3 \mathrm{C} 66 \mathrm{~A}$ & 35.8000 & 43.0117 \\
\hline 1FGL J0319.7+1847 & RBS 0413 & 49.9658 & 18.7594 \\
\hline 1FGL J0416.8+0107 & 1ES 0414+009 & 64.2184 & 1.0901 \\
\hline 1FGL J0449.5-4350 & PKS 0447-437 & 72.3529 & -43.8358 \\
\hline 1FGL J0507.9+6738 & 1ES $0502+675$ & 76.9842 & 67.6233 \\
\hline 1FGL J0521.7+2114 & VER J0521+211 ${ }^{\mathrm{b}}$ & 80.4792 & 21.1900 \\
\hline 1FGL J0710.6+5911 & RGB J0710+591 & 107.6254 & 59.1390 \\
\hline 1FGL J0721.9+7120 & S5 0716+714 & 110.4725 & 71.3433 \\
\hline 1FGL J0809.5+5219 & $1 \mathrm{ES} 0806+524$ & 122.4550 & 52.3161 \\
\hline 1FGL J1015.1+4927 & $1 \mathrm{ES} 1011+496$ & 153.7671 & 49.4336 \\
\hline 1FGL J1103.7-2329 & 1ES $1101-232$ & 165.9083 & -23.4919 \\
\hline 1FGL J1104.4+3812 & Mkn 421 & 166.1138 & 38.2089 \\
\hline 1FGL J1136.6+7009 & Mkn 180 & 174.1100 & 70.1575 \\
\hline 1FGL J1221.3+3008 & 1ES $1218+304$ & 185.3413 & 30.1769 \\
\hline 1FGL J1230.8+1223 & M 87 & 187.7058 & 12.3911 \\
\hline 1FGL J1221.5+2814 & W Com & 185.3821 & 28.2331 \\
\hline 1FGL J1256.2-0547 & $3 \mathrm{C} 279$ & 194.0463 & -5.7894 \\
\hline 1FGL J1325.6-4300 & Cen A & 201.3667 & -43.0183 \\
\hline 1FGL J1426.9+2347 & PKS $1424+240$ & 216.7516 & 23.8000 \\
\hline 1FGL J1428.7+4239 & H $1426+428$ & 217.1358 & 42.6725 \\
\hline 1FGL J1555.7+1111 & PG $1553+113$ & 238.9292 & 11.1900 \\
\hline 1FGL J1653.9+3945 & Mkn 501 & 253.4675 & 39.7600 \\
\hline 1FGL J2000.0+6508 & 1ES $1959+650$ & 299.9996 & 65.1486 \\
\hline 1FGL J2009.5-4849 & PKS 2005-489 & 302.3721 & -48.8219 \\
\hline 1FGL J2158.8-3013 & PKS 2155-304 & 329.7196 & -30.2217 \\
\hline 1FGL J2202.8+4216 & BL Lac & 330.6804 & 42.2778 \\
\hline 1FGL J2347.1+5142 & $1 \mathrm{ES} 2344+514^{\mathrm{b}}$ & 356.7700 & 51.7050 \\
\hline 1FGL J2359.0-3035 & H 2356-309 & 359.7875 & -30.6228 \\
\hline
\end{tabular}

${ }^{a} \mathrm{~J} 2000$ coordinate, in degrees, from
(http://tevcat.uchicago.edu/ .

${ }^{\mathrm{b}}$ This source is at low Galactic latitude $\left(|b|<10^{\circ}\right)$ and is thus not formally in the 1LAC but appears in Table 2 . 
Table 6. Positional coincidences of 1LAC sources with EGRET/AGILE sources

\begin{tabular}{|c|c|c|c|c|c|c|c|c|c|}
\hline 1FGL name & 3EG name & EGR name & GEV name & 1AGL name & LAT flux ${ }^{a}$ & LAT ph. index & $3 \mathrm{EG}$ flux ${ }^{\mathrm{a}}$ & EGRET ph. index & Probable class \\
\hline 1FGL J0204.5+1516 & $3 \mathrm{EG} \mathrm{J0204+1458}$ & EGR J0204+1505 & & & $1.79 \pm 0.58$ & $2.47 \pm 0.18$ & $23.6 \pm 5.6$ & $2.23 \pm 0.28$ & AGN \\
\hline 1FGL J0210.6-5101 & 3EG J0210-5055 & EGR J0210-5058 & GEV J0210-5053 & & $14.59 \pm 0.96$ & $2.37 \pm 0.04$ & $85.5 \pm 4.5$ & $1.99 \pm 0.05$ & BL Lac \\
\hline 1FGL J0222.6+4302 & $3 \mathrm{EG} \mathrm{J0222+4253}$ & EGR J0223+4300 & GEV J0223+4254 & & $21.45 \pm 0.97$ & $1.93 \pm 0.02$ & $18.7 \pm 2.9$ & $2.01 \pm 0.14$ & BL Lac \\
\hline 1FGL J0238.6+1637 & 3EG J0237+1635 & & GEV J0237+1648 & & $43.37 \pm 1.07$ & $2.14 \pm 0.02$ & $65.1 \pm 8.8$ & $1.85 \pm 0.12$ & BL Lac \\
\hline 1FGL J0339.2-0143 & $3 \mathrm{EG}$ J0340-0201 & EGR J0338-0203 & & & $4.00 \pm 0.65$ & $2.50 \pm 0.10$ & $118.8 \pm 22.0$ & $1.84 \pm 0.25$ & FSRQ \\
\hline 1FGL J0416.5-1851 & 3EG J0412-1853 & EGR J0413-1851 & & & $3.59 \pm 0.58$ & $2.37 \pm 0.10$ & $49.5 \pm 16.1$ & $3.25 \pm 0.68$ & FSRQ \\
\hline 1FGL J0423.2-0118 & 3EG J0422-0102 & & & & $13.99 \pm 0.90$ & $2.42 \pm 0.04$ & $50.2 \pm 10.4$ & $2.44 \pm 0.19$ & FSRQ \\
\hline 1FGL J0433.5+2905 & $3 \mathrm{EG} \mathrm{J0433+2908}$ & EGR J0433+2906 & GEV J0433+2907 & & $6.57 \pm 0.83$ & $2.13 \pm 0.06$ & $22.0 \pm 2.8$ & $1.90 \pm 0.10$ & BL Lac \\
\hline 1FGL J0442.7-0019 & 3EG J0442-0033 & EGR J0442-0027 & GEV J0441-0044 & & $17.27 \pm 0.91$ & $2.44 \pm 0.04$ & $79.0 \pm 10.1$ & $2.37 \pm 0.18$ & FSRQ \\
\hline 1FGL J0448.6+1118 & $3 \mathrm{EG}$ J0450+1105 & & & & $5.90 \pm 0.88$ & $2.51 \pm 0.09$ & $109.5 \pm 19.4$ & $2.27 \pm 0.16$ & BL Lac \\
\hline 1FGL J0455.6-4618 & 3EG J0458-4635 & & & & $6.15 \pm 0.83$ & $2.57 \pm 0.09$ & $7.7 \pm \quad 2.1$ & $2.75 \pm 0.35$ & FSRQ \\
\hline 1FGL J0457.0-2325 & 3EG J0456-2338 & EGR J0456-2334 & & & $51.14 \pm 1.07$ & $2.21 \pm 0.02$ & $14.7 \pm 4.2$ & $3.14 \pm 0.47$ & FSRQ \\
\hline 1FGL J0501.0-0200 & 3EG J0500-0159 & & & & $3.74 \pm 0.70$ & $2.50 \pm 0.11$ & $11.2 \pm 2.3$ & $2.45 \pm 0.27$ & FSRQ \\
\hline 1FGL J0509.3+0540 & & EGR J0509+0550 & GEV J0508+0540 & & $5.77 \pm 0.73$ & $2.16 \pm 0.06$ & & & BL Lac \\
\hline 1FGL J0516.7-6207 & 3EG J0512-6150 & & & & $5.76 \pm 0.95$ & $2.28 \pm 0.09$ & $7.2 \pm 1.7$ & $2.40 \pm 0.26$ & $\mathrm{XXX}$ \\
\hline 1FGL J0531.0+1331 & $3 \mathrm{EG}$ J0530+1323 & EGR J0530+1331 & GEV J0530+1340 & & $16.85 \pm 1.28$ & $2.64 \pm 0.06$ & $93.5 \pm 3.6$ & $2.46 \pm 0.04$ & FSRQ \\
\hline 1FGL J0538.8-4404 & 3EG J0540-4402 & EGR J0540-4358 & GEV J0540-4359 & 1AGL J0538-4424 & $37.77 \pm 1.06$ & $2.27 \pm 0.02$ & $25.3 \pm 3.1$ & $2.41 \pm 0.12$ & BL Lac \\
\hline 1FGL J0540.9-0547 & 3EG J0542-0655 & & & & $3.73 \pm 1.01$ & $2.37 \pm 0.12$ & $66.5 \pm 19.5$ & & FSRQ \\
\hline 1FGL J0721.9+7120 & $3 \mathrm{EG} \mathrm{J0721+7120}$ & EGR J0723+7134 & GEV J0719+7133 & 1AGL J0722+7125 & $17.26 \pm 0.75$ & $2.15 \pm 0.03$ & $17.8 \pm 2.0$ & $2.19 \pm 0.11$ & BL Lac \\
\hline 1FGL J0738.2+1741 & $3 \mathrm{EG}$ J0737+1721 & EGR J0737+1720 & & & $4.60 \pm 0.48$ & $2.02 \pm 0.06$ & $16.4 \pm 3.3$ & $2.60 \pm 0.28$ & BL Lac \\
\hline 1FGL J0742.2+5443 & $3 \mathrm{EG} \mathrm{J0743+5447}$ & EGR J0743+5438 & & & $5.22 \pm 0.75$ & $2.45 \pm 0.09$ & $30.3 \pm 5.0$ & $2.03 \pm 0.20$ & FSRQ \\
\hline 1FGL J0830.5+2407 & 3EG J0829+2413 & EGR J0829+2415 & & & $8.35 \pm 0.84$ & $2.79 \pm 0.09$ & $24.9 \pm 3.9$ & $2.42 \pm 0.21$ & FSRQ \\
\hline 1FGL J0831.6+0429 & & EGR J0829+0510 & & & $7.35 \pm 0.76$ & $2.50 \pm 0.07$ & & & BL Lac \\
\hline 1FGL J0842.2+7054 & $3 \mathrm{EG}$ J0845+7049 & & & & $8.06 \pm 1.04$ & $2.98 \pm 0.12$ & $10.2 \pm 1.8$ & $2.62 \pm 0.16$ & FSRQ \\
\hline 1FGL J0850.0-1213 & $3 \mathrm{EG}$ J0852-1216 & EGR J0852-1224 & & & $5.02 \pm 0.64$ & $2.27 \pm 0.07$ & $44.4 \pm 11.6$ & $1.58 \pm 0.58$ & FSRQ \\
\hline 1FGL J0854.8+2006 & 3EG J0853+1941 & EGR J0853+2015 & & & $7.03 \pm 0.82$ & $2.38 \pm 0.07$ & $10.6 \pm 3.0$ & $2.03 \pm 0.35$ & BL Lac \\
\hline 1FGL J0957.7+5523 & & EGR J0957+5513 & GEV J0956+5508 & & $11.39 \pm 0.62$ & $2.05 \pm 0.03$ & & & FSRQ \\
\hline 1FGL J1000.1+6539 & 3EG J0958+6533 & EGR J0956+6524 & & & $2.59 \pm 0.67$ & $2.51 \pm 0.16$ & $15.4 \pm 3.0$ & $2.08 \pm 0.24$ & BL Lac \\
\hline 1FGL J1104.4+3812 & $3 \mathrm{EG} \mathrm{J1104+3809}$ & EGR J1104+3813 & GEV J1104+3809 & 1AGL J1104+3754 & $16.93 \pm 0.58$ & $1.81 \pm 0.02$ & $13.9 \pm 1.8$ & $1.57 \pm 0.15$ & BL Lac \\
\hline 1FGL J1133.1+0033 & 3EG J1133+0033 & & & & $2.71 \pm 0.53$ & $2.18 \pm 0.11$ & $10.6 \pm 3.0$ & $2.73 \pm 0.63$ & BL Lac \\
\hline 1FGL J1159.4+2914 & $3 \mathrm{EG} \mathrm{J1200+2847}$ & & GEV J1201+2906 & & $12.24 \pm 0.74$ & $2.37 \pm 0.04$ & $50.9 \pm 11.9$ & $1.98 \pm 0.22$ & FSRQ \\
\hline 1FGL J1221.5+2814 & & & & 1AGL J1222+2851 & $7.82 \pm 0.61$ & $2.06 \pm 0.04$ & & & BL Lac \\
\hline 1FGL J1224.7+2121 & $3 \mathrm{EG} \mathrm{J} 1224+2118$ & & & & $8.07 \pm 0.75$ & $2.55 \pm 0.07$ & $13.9 \pm 1.8$ & $2.28 \pm 0.13$ & FSRQ \\
\hline 1FGL J1225.8+4336 & $3 \mathrm{EG} \mathrm{J} 1227+4302$ & & & & $2.82 \pm 0.69$ & $2.81 \pm 0.18$ & $21.7 \pm 7.1$ & & $\mathrm{Xxx}$ \\
\hline 1FGL J1229.1+0203 & $3 \mathrm{EG}$ J1229+0210 & EGR J1229+0203 & & 1AGL J1228+0142 & $55.30 \pm 1.48$ & $2.75 \pm 0.03$ & $15.4 \pm 1.8$ & $2.58 \pm 0.09$ & FSRQ \\
\hline 1FGL J1239.5+0443 & & EGR J1237+0434 & & 1AGL J1238+0406 & $8.41 \pm 0.75$ & $2.35 \pm 0.06$ & & & FSRQ \\
\hline 1FGL J1256.2-0547 & 3EG J1255-0549 & EGR J1256-0552 & GEV J1256-0546 & 1AGL J1256-0549 & $68.84 \pm 1.37$ & $2.32 \pm 0.02$ & $179.7 \pm 6.7$ & $1.96 \pm 0.04$ & FSRQ \\
\hline 1FGL J1258.7-2221 & & EGR J1259-2209 & & & $5.66 \pm 0.80$ & $2.39 \pm 0.08$ & & & FSRQ \\
\hline 1FGL J1321.1+2214 & $3 \mathrm{EG} \mathrm{J} 1323+2200$ & & & & $1.62 \pm 0.50$ & $2.21 \pm 0.15$ & $18.1 \pm 4.0$ & $1.86 \pm 0.35$ & FSRQ \\
\hline 1FGL J1325.6-4300 & 3EG J1324-4314 & & & & $20.40 \pm 1.47$ & $2.71 \pm 0.06$ & $13.6 \pm 2.5$ & $2.58 \pm 0.26$ & AGN \\
\hline 1FGL J1337.7-1255 & & EGR J1337-1310 & & & $7.21 \pm 1.12$ & $2.50 \pm 0.09$ & & & FSRQ \\
\hline 1FGL J1408.9-0751 & 3EG J1409-0745 & EGR J1409-0736 & GEV J1409-0741 & & $5.21 \pm 0.71$ & $2.42 \pm 0.08$ & $97.6 \pm 9.1$ & $2.29 \pm 0.11$ & FSRQ \\
\hline 1FGL J1422.7+3743 & $3 \mathrm{EG} \mathrm{J1424+3734}$ & EGR J1424+3730 & & & $2.36 \pm 0.72$ & $2.63 \pm 0.19$ & $16.3 \pm 4.9$ & $3.25 \pm 0.46$ & BL Lac \\
\hline 1FGL J1428.2-4204 & 3EG J1429-4217 & EGR J1428-4240 & & & $6.70 \pm 0.82$ & $2.31 \pm 0.07$ & $29.5 \pm 5.3$ & $2.13 \pm 0.21$ & FSRQ \\
\hline 1FGL J1457.5-3540 & 3EG J1500-3509 & & & & $33.27 \pm 1.18$ & $2.27 \pm 0.02$ & $10.9 \pm 2.8$ & $2.99 \pm 0.37$ & FSRQ \\
\hline 1FGL J1503.5-1544 & 3EG J1504-1537 & EGR J1504-1539 & & & $0.76 \pm 0.38$ & $1.74 \pm 0.19$ & $33.2 \pm 10.3$ & & BL Lac \\
\hline 1FGL J1505.1-3435 & 3EG J1500-3509 & & & & $1.11 \pm 0.54$ & $2.02 \pm 0.19$ & $10.9 \pm 2.8$ & $2.99 \pm 0.37$ & $\mathrm{xxx}$ \\
\hline 1FGL J1512.8-0906 & 3EG J1512-0849 & EGR J1512-0857 & & 1AGL J1511-0908 & $127.10 \pm 1.85$ & $2.41 \pm 0.01$ & $18.0 \pm 3.8$ & $2.47 \pm 0.21$ & FSRQ \\
\hline 1FGL J1607.1+1552 & $3 \mathrm{EG} \mathrm{J1605+1553}$ & EGR J1607+1533 & & & $3.86 \pm 0.59$ & $2.25 \pm 0.08$ & $42.0 \pm 12.3$ & $2.06 \pm 0.41$ & AGN \\
\hline 1FGL J1609.0+1031 & $3 \mathrm{EG} \mathrm{J1608+1055}$ & EGR J1608+1051 & & & $6.19 \pm 0.87$ & $2.72 \pm 0.10$ & $34.9 \pm 5.6$ & $2.63 \pm 0.24$ & FSRQ \\
\hline 1FGL J1613.5+3411 & $3 \mathrm{EG} \mathrm{J1614+3424}$ & & GEV J1613+3432 & & $1.02 \pm 0.48$ & $2.29 \pm 0.22$ & $26.5 \pm 4.0$ & $2.42 \pm 0.15$ & FSRQ \\
\hline
\end{tabular}


Table 6 - Continued

\begin{tabular}{|c|c|c|c|c|c|c|c|c|c|}
\hline 1FGL name & 3EG name & EGR name & GEV name & 1AGL name & LAT flux ${ }^{a}$ & LAT ph. index & $3 \mathrm{EG}$ flux $^{\mathrm{a}}$ & EGRET ph. index & Probable class \\
\hline 1FGL J1625.7-2524 & 3EG J1626-2519 & & GEV J1626-2502 & & $10.53 \pm 1.42$ & $2.36 \pm 0.06$ & $42.6 \pm 6.6$ & $2.21 \pm 0.13$ & FSRQ \\
\hline 1FGL J1626.2-2956 & 3EG J1625-2955 & EGR J1625-2958 & GEV J1626-2955 & & $5.02 \pm 0.94$ & $2.36 \pm 0.10$ & $258.9 \pm 15.3$ & $2.07 \pm 0.07$ & FSRQ \\
\hline 1FGL J1635.0+3808 & $3 \mathrm{EG}$ J1635+3813 & & GEV J1636+3812 & & $19.21 \pm 1.17$ & $2.47 \pm 0.04$ & $107.5 \pm 9.6$ & $2.15 \pm 0.09$ & FSRQ \\
\hline 1FGL J1635.4+8228 & $3 \mathrm{EG} \mathrm{J} 1621+8203$ & & & & $3.78 \pm 0.71$ & $2.50 \pm 0.12$ & $10.4 \pm 3.0$ & $2.29 \pm 0.49$ & AGN \\
\hline 1FGL J1702.7-6217 & 3EG J1659-6251 & & & & $4.74 \pm 1.04$ & $2.54 \pm 0.13$ & $47.0 \pm 13.1$ & $2.54 \pm 0.37$ & FSRQ \\
\hline 1FGL J1728.2+0431 & $3 \mathrm{EG}$ J1727+0429 & EGR J1727+0416 & & & $6.66 \pm 1.05$ & $2.65 \pm 0.11$ & $17.9 \pm 4.1$ & $2.67 \pm 0.26$ & FSRQ \\
\hline 1FGL J1733.0-1308 & 3EG J1733-1313 & & & & $8.73 \pm 1.12$ & $2.34 \pm 0.07$ & $36.1 \pm 3.4$ & $2.23 \pm 0.10$ & FSRQ \\
\hline 1FGL J1740.0+5209 & $3 \mathrm{EG} \mathrm{J1738+5203}$ & EGR J1740+5213 & & & $17.20 \pm 1.03$ & $2.71 \pm 0.05$ & $18.2 \pm 3.5$ & $2.42 \pm 0.23$ & FSRQ \\
\hline 1FGL J1849.3+6705 & & & & 1AGL J1846+6714 & $24.24 \pm 0.89$ & $2.25 \pm 0.03$ & & & FSRQ \\
\hline 1FGL J1911.2-2007 & 3EG J1911-2000 & EGR J1912-2000 & & & $12.37 \pm 1.07$ & $2.42 \pm 0.05$ & $17.5 \pm 2.7$ & $2.39 \pm 0.18$ & FSRQ \\
\hline 1FGL J2006.6-2302 & $3 \mathrm{EG}$ J2006-2321 & & & & $7.16 \pm 0.95$ & $2.68 \pm 0.10$ & $19.8 \pm 4.4$ & $2.33 \pm 0.36$ & FSRQ \\
\hline 1FGL J2009.5-4849 & & & GEV J2009-4827 & & $3.94 \pm 0.49$ & $1.90 \pm 0.06$ & & & BL Lac \\
\hline 1FGL J2025.6-0735 & 3EG J2025-0744 & & GEV J2024-0812 & 1AGL J2026-0732 & $29.15 \pm 1.18$ & $2.35 \pm 0.03$ & $74.5 \pm 13.4$ & $2.38 \pm 0.17$ & FSRQ \\
\hline 1FGL J2031.5+1219 & & EGR J2032+1226 & & & $4.06 \pm 0.90$ & $2.41 \pm 0.12$ & & & BL Lac \\
\hline 1FGL J2035.4+1100 & $3 \mathrm{EG} \mathrm{J} 2036+1132$ & & & & $7.74 \pm 1.11$ & $2.68 \pm 0.10$ & $13.3 \pm 3.1$ & $2.83 \pm 0.26$ & FSRQ \\
\hline 1FGL J2056.3-4714 & 3EG J2055-4716 & & & & $17.00 \pm 1.03$ & $2.54 \pm 0.05$ & $23.6 \pm 6.0$ & $2.04 \pm 0.35$ & FSRQ \\
\hline 1FGL J2158.8-3013 & 3EG J2158-3023 & EGR J2200-3015 & & & $21.44 \pm 0.70$ & $1.91 \pm 0.02$ & $30.4 \pm \quad 7.7$ & $2.35 \pm 0.26$ & BL Lac \\
\hline 1FGL J2202.8+4216 & 3 EG J2202+4217 & EGR J2204+4225 & & & $16.81 \pm 1.01$ & $2.38 \pm 0.04$ & $39.9 \pm 11.6$ & $2.60 \pm 0.28$ & BL Lac \\
\hline 1FGL J2212.1+2358 & $3 \mathrm{EG} \mathrm{J} 2209+2401$ & & & & $1.19 \pm 0.50$ & $2.13 \pm 0.19$ & $14.6 \pm \quad 4.2$ & $2.48 \pm 0.50$ & FSRQ \\
\hline 1FGL J2212.9+0654 & & EGRc J2215+0653 & & & $3.01 \pm 0.61$ & $2.33 \pm 0.11$ & & & FSRQ \\
\hline 1FGL J2232.5+1144 & $3 \mathrm{EG} \mathrm{J} 2232+1147$ & & & & $14.70 \pm 0.97$ & $2.56 \pm 0.05$ & $19.2 \pm 2.8$ & $2.45 \pm 0.14$ & FSRQ \\
\hline 1FGL J2235.7-4817 & & EGR J2233-4812 & & & $2.02 \pm 0.56$ & $2.43 \pm 0.17$ & & & FSRQ \\
\hline 1FGL J2253.9+1608 & $3 \mathrm{EG} \mathrm{J2254+1601}$ & EGR J2253+1606 & GEV J $2253+1622$ & 1AGL J2254+1602 & $136.81 \pm 1.74$ & $2.47 \pm 0.01$ & $53.7 \pm 4.0$ & $2.21 \pm 0.06$ & FSRQ \\
\hline 1FGL J2258.0-2757 & & EGR J2258-2745 & & & $5.61 \pm 0.85$ & $2.67 \pm 0.10$ & $\ldots$ & $\ldots$ & FSRQ \\
\hline 1FGL J2323.5-0315 & 3EG J2321-0328 & & & & $6.05 \pm 0.80$ & $2.45 \pm 0.08$ & $38.2 \pm 10.1$ & & FSRQ \\
\hline
\end{tabular}

${ }^{a}$ In units of $10^{-8}$ photons $\mathrm{cm}^{-2} \mathrm{~s}^{-1}$.

Note. - Associated sources from the 3rd EGRET (3EG; Hartman et al. 1999), Revised EGRET (EGR; Casandiian \& Grenier 2008), High-energy EGRET (GEV; Lamb \& Macomb 1997), and one-year AGILE (1AGL; Pittori et al.|2009) catalogs. The LAT and EGRET fluxes and spectral indices are also provided. 
Table 7. Positional associations with hard X-ray sources

\begin{tabular}{|c|c|c|c|c|}
\hline LAT Source & Hard X-ray Source & $\mathrm{RA}^{\mathrm{a}}$ & $\mathrm{DEC}^{\mathrm{a}}$ & Type \\
\hline 1FGL J0217.8+7353 & $1 \mathrm{ES} 0212+735$ & 34.32489 & 73.822807 & FSRQ \\
\hline 1FGL J0319.7+4130 & NGC 1275 & 49.950871 & 41.501099 & AGN \\
\hline 1FGL J0325.0+3403 & B2 $0321+33 B$ & 51.18626 & 34.176857 & AGN \\
\hline 1FGL J0334.2+3233 & NRAO 140 & 54.128605 & 32.305359 & FSRQ \\
\hline 1FGL J0405.6-1309 & PKS $0403-13$ & 61.39616 & -13.142807 & FSRQ \\
\hline 1FGL J0522.8-3632 & PKS $0521-36$ & 80.746483 & -36.458878 & BLL \\
\hline 1FGL J0531.0+1331 & PKS $0528+134$ & 82.754601 & 13.562002 & FSRQ \\
\hline 1FGL J0538.8-4404 & PKS $0537-441$ & 84.750519 & -44.104771 & BLL \\
\hline 1FGL J0539.1-2847 & PKS $0537-286$ & 84.951698 & -28.645336 & FSRQ \\
\hline 1FGL J0636.1-7521 & PKS $0637-75$ & 99.080544 & -75.244789 & FSRQ \\
\hline 1FGL J0710.6+5911 & BZB J0710+5908 & 107.664192 & 59.14875 & BLL \\
\hline 1FGL J0746.6+2548 & B2 $0743+25$ & 116.609474 & 25.811897 & FSRQ \\
\hline 1FGL J0750.6+1235 & PKS $0748+126$ & 117.666161 & 12.520621 & FSRQ \\
\hline 1FGL J0806.2+6148 & CGRaBS J0805+6144 & 121.314117 & 61.73798 & FSRQ \\
\hline 1FGL J0842.2+7054 & $4 \mathrm{C}+71.07$ & 130.395554 & 70.889877 & FSRQ \\
\hline 1FGL J0949.0+0021 & CGRaBS J0948+0022 & 147.228745 & 0.350572 & FSRQ \\
\hline 1FGL J0956.5+6938 & M 82 & 148.94429 & 69.694511 & AGN \\
\hline 1FGL J1048.7+8054 & CGRaBS J1044+8054 & 161.060471 & 80.926788 & FSRQ \\
\hline 1FGL J1103.7-2329 & CRATES J1103-2329 & 165.899918 & -23.477991 & BLL \\
\hline 1FGL J1104.4+3812 & Mkn 421 & 166.116058 & 38.208893 & BLL \\
\hline 1FGL J1130.2-1447 & PKS 1127-14 & 172.528671 & -14.813811 & FSRQ \\
\hline 1FGL J1136.2+6739 & BZB J1136+6737 & 174.020081 & 67.657433 & BLL \\
\hline 1FGL J1221.3+3008 & B2 $1218+30$ & 185.328064 & 30.148579 & BLL \\
\hline 1FGL J1222.5+0415 & $4 \mathrm{C}+04.42$ & 185.589111 & 4.235839 & FSRQ \\
\hline 1FGL J1224.7+2121 & $4 \mathrm{C}+21.35$ & 186.224121 & 21.383167 & FSRQ \\
\hline 1FGL J1229.1+0203 & 3C 273 & 187.27565 & 2.043433 & FSRQ \\
\hline 1FGL J1256.2-0547 & 3C 279 & 194.041 & -5.803322 & FSRQ \\
\hline 1FGL J1305.4-4928 & NGC 4945 & 196.353302 & -49.473759 & AGN \\
\hline 1FGL J1307.0-4030 & ESO $323-G 77$ & 196.643127 & -40.422039 & AGN \\
\hline 1FGL J1325.6-4300 & Cen A & 201.362106 & -43.026054 & AGN \\
\hline 1FGL J1331.9-0506 & PKS $1329-049$ & 203.010651 & -5.174589 & FSRQ \\
\hline 1FGL J1417.8+2541 & $2 \mathrm{E} 1415+2557$ & 214.479584 & 25.733086 & BLL \\
\hline 1FGL J1428.7+4239 & $1 \mathrm{ES} 1426+428$ & 217.147217 & 42.666458 & BLL \\
\hline 1FGL J1442.8+1158 & $1 \mathrm{ES} 1440+122$ & 220.704819 & 12.045237 & BLL \\
\hline 1FGL J1512.8-0906 & PKS $1510-08$ & 228.205551 & -9.086402 & FSRQ \\
\hline 1FGL J1517.8-2423 & AP Lib & 229.457718 & -24.370974 & BLL \\
\hline 1FGL J1555.7+1111 & PG $1553+113$ & 238.886841 & 11.209181 & BLL \\
\hline 1FGL J1626.2-2956 & PKS $1622-29$ & 246.539917 & -29.818855 & FSRQ \\
\hline 1FGL J1653.9+3945 & Mkn 501 & 253.446915 & 39.768932 & BLL \\
\hline 1FGL J1829.8+4845 & $3 \mathrm{C} 380$ & 277.403839 & 48.753803 & AGN \\
\hline 1FGL J1925.2-2919 & PKS B1921-293 & 291.157959 & -29.235388 & FSRQ \\
\hline 1FGL J1938.2-3957 & PKS $1933-400^{\mathrm{b}}$ & 294.294 & -39.932 & FSRQ \\
\hline 1FGL J2000.0+6508 & $1 \mathrm{ES} 1959+650$ & 299.94632 & 65.150742 & BLL \\
\hline 1FGL J2148.5+0654 & $4 \mathrm{C}+06.69$ & 327.031952 & 6.948918 & FSRQ \\
\hline 1FGL J2202.8+4216 & BL Lac & 330.72821 & 42.273628 & BLL \\
\hline 1FGL J2229.7-0832 & PKS 2227-08 & 337.407898 & -8.519718 & FSRQ \\
\hline 1FGL J2232.5+1144 & CTA 102 & 338.135529 & 11.73317 & FSRQ \\
\hline 1FGL J2253.9+1608 & 3C 454.3 & 343.483856 & 16.153084 & FSRQ \\
\hline 1FGL J2327.7+0943 & PKS $2325+093$ & 351.892822 & 9.633142 & FSRQ \\
\hline 1FGL J2359.0-3035 & 1H $2351-315$ & 359.779633 & -30.594545 & BLL \\
\hline
\end{tabular}

${ }^{\mathrm{a}} \mathrm{J} 2000$ coordinate, in degrees, from the 54-month Palermo BAT catalog where available or from the fourth IBIS catalog otherwise.

${ }^{\mathrm{b}}$ Hard X-ray source found only in the fourth IBIS catalog. 